PRELIMINARY INTERPRETIVE REPORT 2017-2

\title{
GEOLOGIC MAP OF THE CASTLE MOUNTAIN-CARIBOU FAULT SYSTEM, TALKEETNA MOUNTAINS, ALASKA
}

by

William A. Fuchs, Ph.D.

2019

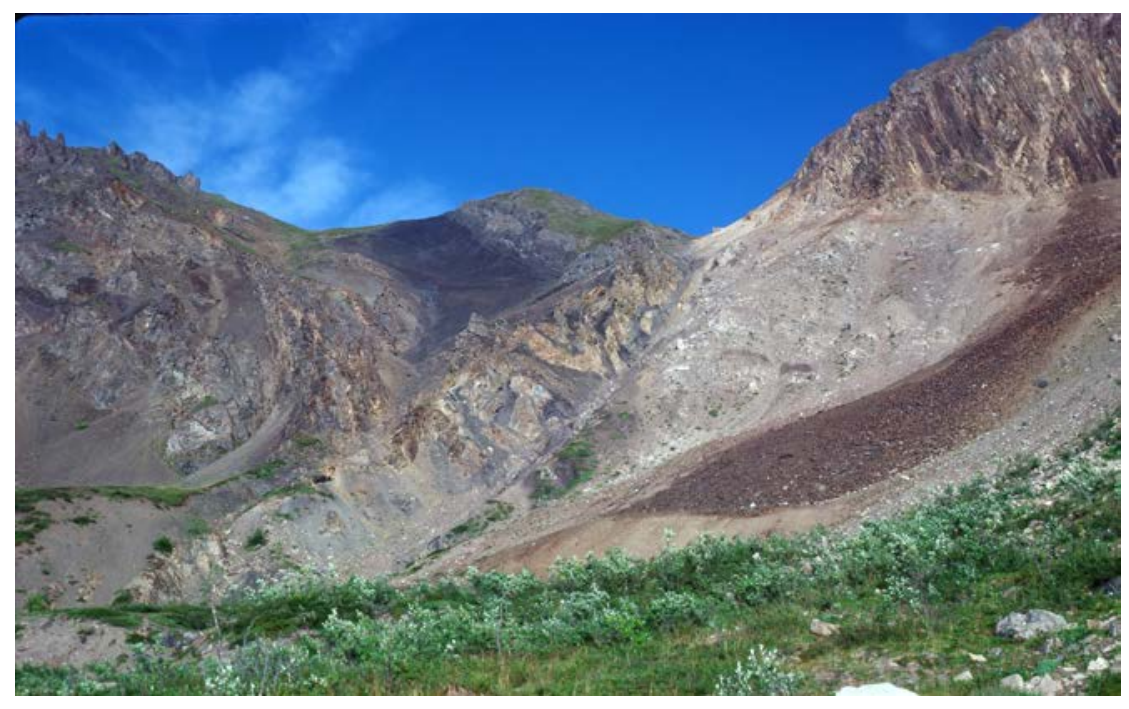

Released by

STATE OF ALASKA

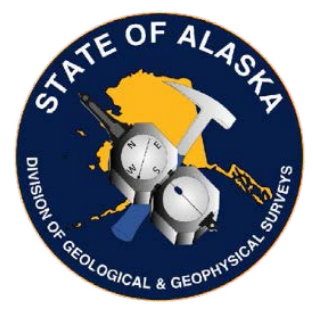

DEPARTMENT OF NATURAL RESOURCES

Division of Geological \& Geophysical Surveys

3354 College Road, Fairbanks, Alaska 99709-3707

Phone: (907) 451-5010, Fax (907) 451-5050

dggspubs@alaska.gov

dggs.alaska.gov

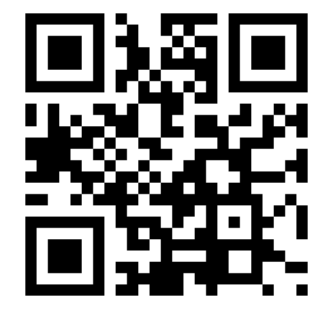




\section{STATE OF ALASKA}

Michael J. Dunleavy, Governor

\section{DEPARTMENT OF NATURAL RESOURCES}

Corri A. Feige, Commissioner

\section{DIVISION OF GEOLOGICAL \& GEOPHYSICAL SURVEYS}

Steve Masterman, State Geologist and Director

Publications produced by the Division of Geological \& Geophysical

Surveys (DGGS) are available for free download from the DGGS

website (dggs.alaska.gov). Publications on hard-copy or digital

media can be examined or purchased in the Fairbanks office:

\section{Alaska Division of Geological \& Geophysical Surveys}

3354 College Rd., Fairbanks, Alaska 99709-3707

Phone: (907) 451-5010 Fax (907) 451-5050

dggspubs@alaska.gov | dggs.alaska.gov

\section{DGGS publications are also available at:}

Alaska State Library,

Historical Collections \& Talking Book Center

395 Whittier Street

Juneau, Alaska 99811

Alaska Resource Library and Information Services (ARLIS)

3150 C Street, Suite 100

Anchorage, Alaska 99503

\section{Suggested citation:}

Fuchs, W.A., 2017, Geologic map of the Castle Mountain-Caribou fault system, Talkeetna Mountains, Alaska: Alaska Division of Geological \& Geophysical

Surveys Preliminary Interpretive Report 2017-2, 57 p., 2 sheets, scale 1:24,000.

http://doi.org/10.14509/29715

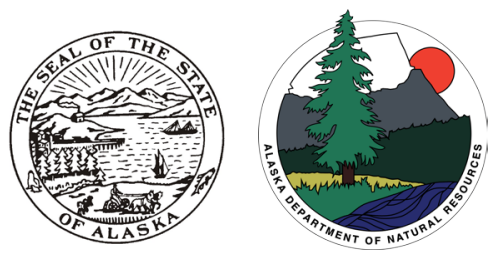




\section{CONTENTS}

INTRODUCTION

FIELD NOTES

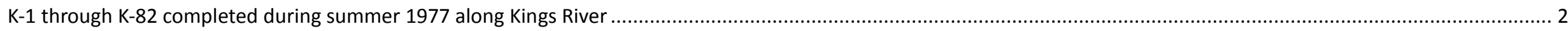

C-1 through C-83 July 1977 Chickaloon River. Camp established near where Doone Creek joins Chickaloon River....................................................................................12

P-1 through P-61 completed August 1977 along Puddingstone Hill - 8/21/77. Camp Elevation: 2,810 feet ..........................................................................................20

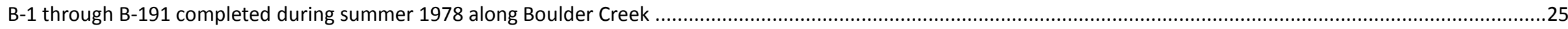

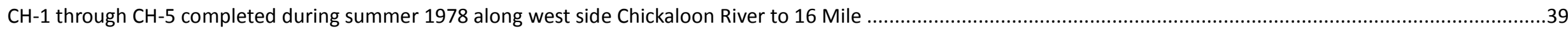

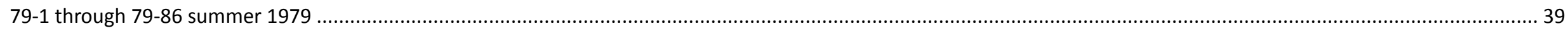

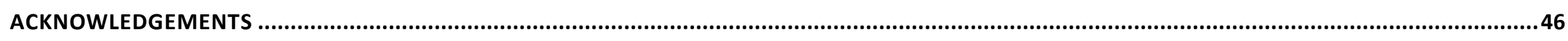

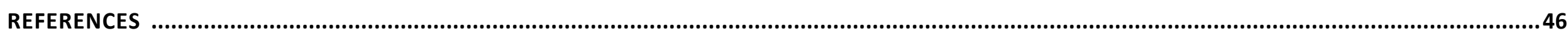

TABLE

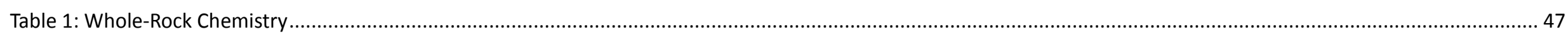

ASSAY CERTIFICATES

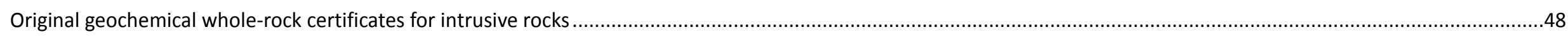

\section{SHEETS}

Sheet 1: Geologic map of the Castle Mountain - Caribou fault system, Talkeetna Mountains, Alaska

Sheet 2: Station locality map of the Castle Mountain - Caribou fault system, Talkeetna Mountains, Alaska

Cover photo: Castle Mountain Splay Fault located at the prominent drainage divide north of Puddingstone Hill, and viewed looking easterly. 


\section{Introduction}

The Castle Mountain fault extends along the southern Talkeetna Mountains range front in south-central Alaska. This publication presents geologic mapping, field notes, and whole-rock major-oxide geochemical analyses of intrusive rocks from an area slightly west of Kings River to considerably east of Boulder Creek, a distance of $39 \mathrm{~km}$ and total map area of 264 square $\mathrm{km}$. The map represents a newly compiled and digitized version of the $1980 \mathrm{Ph} . \mathrm{D}$. dissertation map of William A. Fuchs (University of Utah). This 1:24,000 scale map built upon a previous 1:63,360 scale map by Detterman, R.L., et al., 1976 (Misc. Field Studies Map MF-738). The Fuchs map represents three field seasons of on-the-ground mapping. The author always assumed through the years that other maps would build upon, and perhaps, supplant his work, but no other comprehensive geologic maps of similar scale have appeared in the intervening years, hence, the publication of this "historic" map.

The field notes key to station localities shown on the map, and eastings and northings are UTM NAD27 CONUS. They contain stratigraphic, paleontologic, paleomagnetic, geochemical, petrologic, and structural information, much of which did not make it into the dissertation. The U.S. Geological Survey Alaskan Branch gave initial material support, and three oil companies, Amoco Production Co., Atlantic Richfield Co., and Union Oil Company of California, funded and supported the original project. This area of the Castle Mountain fault has been of interest to oil and coal exploration, and tectonic studies. Because of the proximity to Anchorage and the active nature of the fault, the map area is of particular relevance to earthquake studies. The geochemistry samples were donated to the Geologic Material Center of the Alaska DGGS. The Fuchs dissertation, entitled Tertiary Tectonic History of the Castle Mountain Caribou Fault System, Talkeetna Mountains, Alaska, is available on the Alaska DGGS website. 


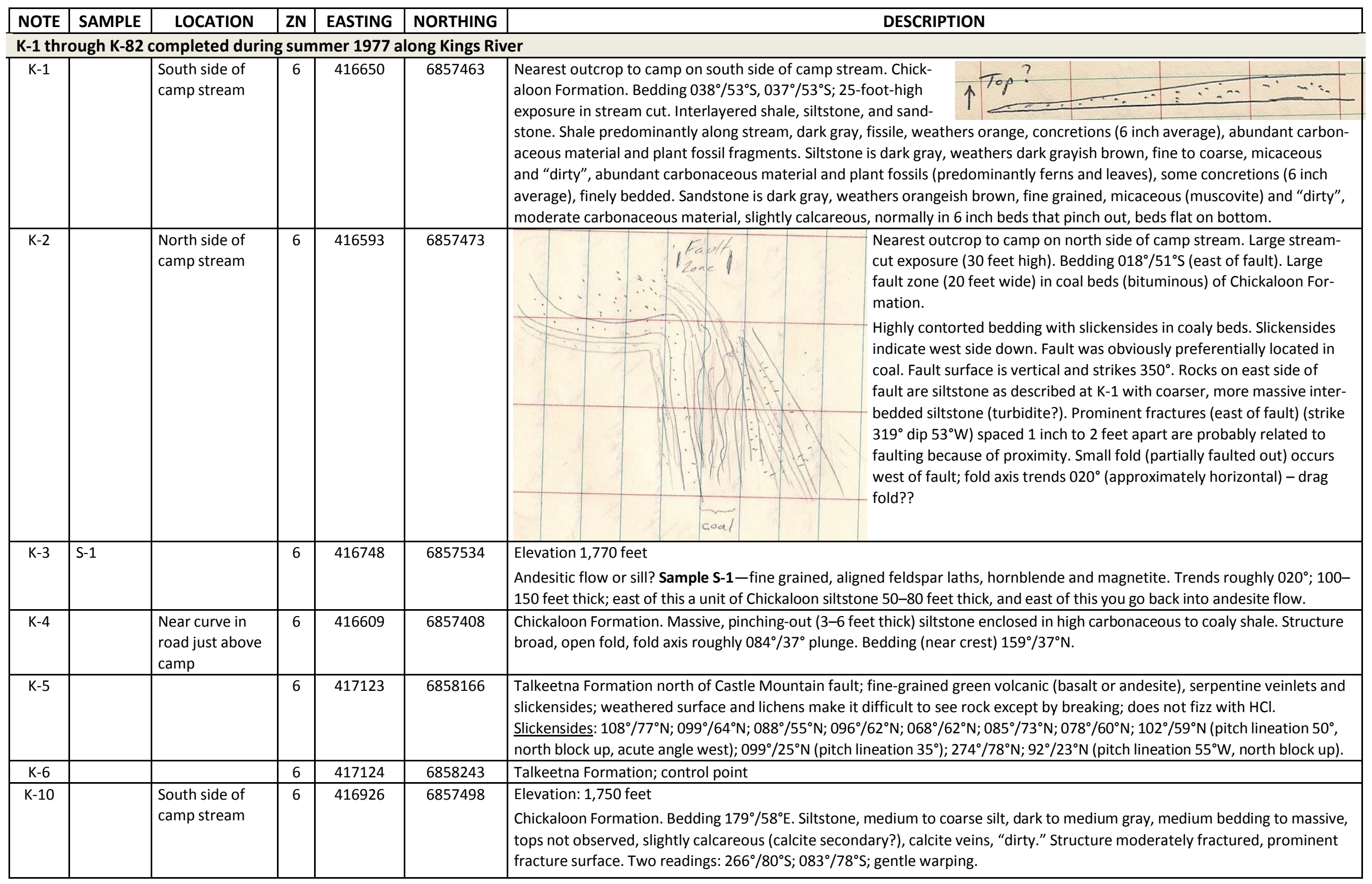


CASTLE MOUNTAIN - CARIBOU FAULT SYSTEM FIELD NOTES (referenced to station localities - UTM NAD27 CONUS)

by William A. Fuchs

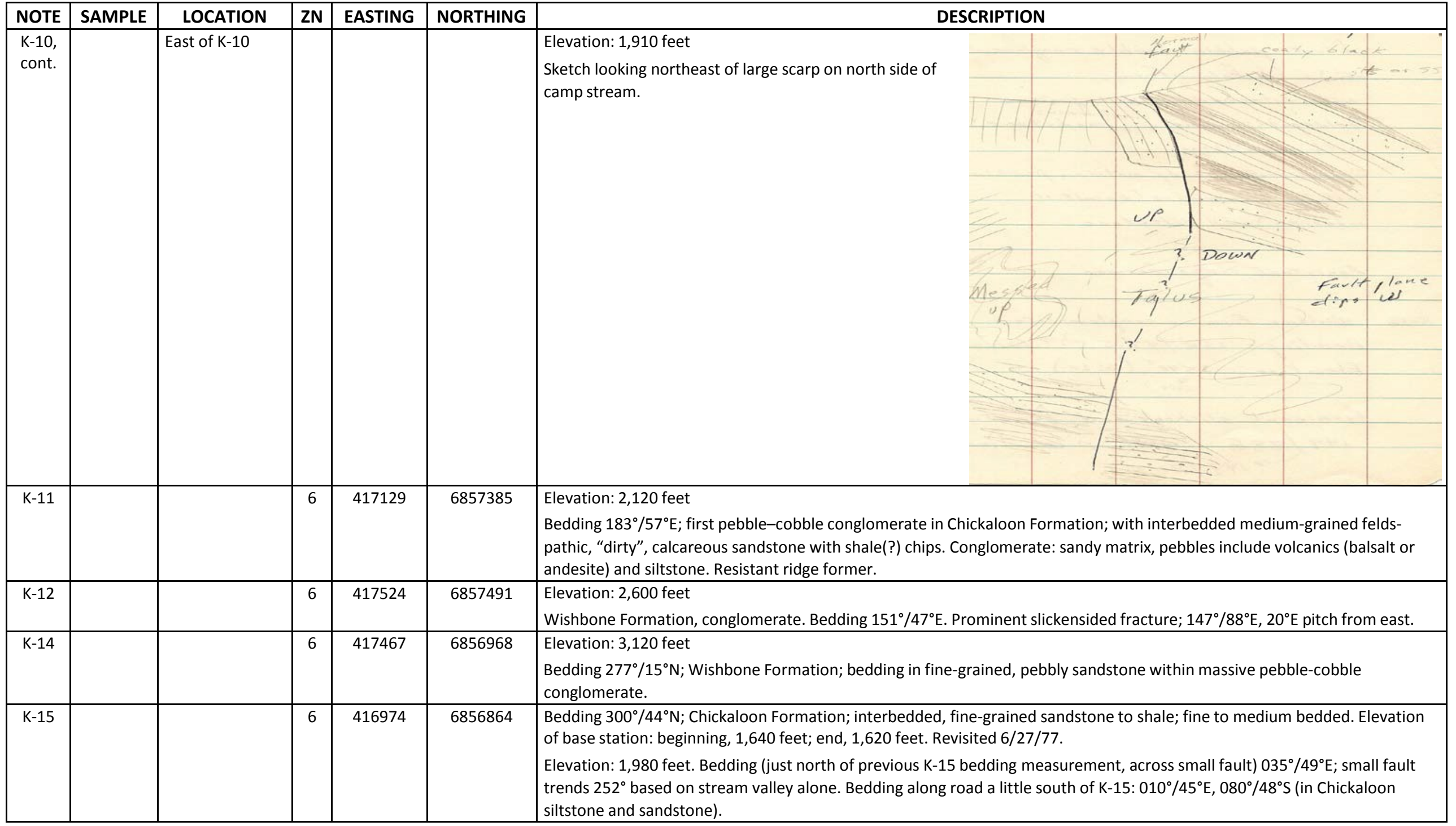


CASTLE MOUNTAIN - CARIBOU FAULT SYSTEM FIELD NOTES (referenced to station localities - UTM NAD27 CONUS)

by William A. Fuchs

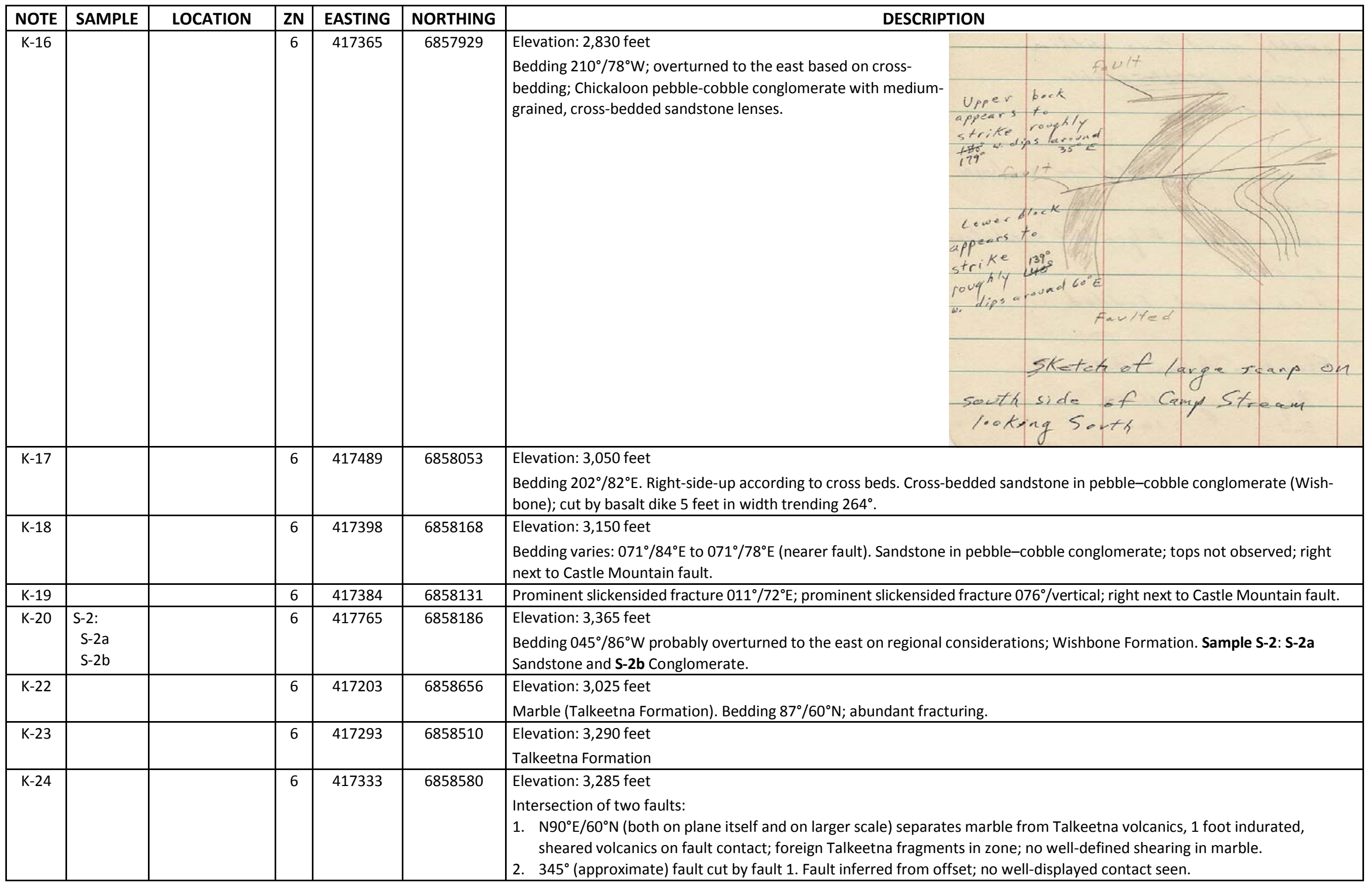


CASTLE MOUNTAIN - CARIBOU FAULT SYSTEM FIELD NOTES (referenced to station localities - UTM NAD27 CONUS)

by William A. Fuchs

\begin{tabular}{|c|c|c|c|c|c|c|c|}
\hline NOTE & SAMPLE & LOCATION & ZN & EASTING & NORTHING & & DESCRIPTION \\
\hline $\mathrm{K}-25$ & & & 6 & 417382 & 6858571 & \multicolumn{2}{|c|}{$\begin{array}{l}\text { Elevation: } 3,500 \text { feet } \\
\text { Fault contact of same fault as fault } 1 \text { at } \mathrm{K}-24 \text {. 3-foot gouge zone in marble; } 3 \text {-foot pyritic altered zone in volcanics. Fold axis } \\
\text { measurements of small folds in gouge (bearing/plunge): } 118^{\circ} / 60^{\circ} ; 145^{\circ} / 63^{\circ} ; 145^{\circ} / 61^{\circ} ; 135^{\circ} / 65^{\circ} ; 145^{\circ} / 43^{\circ} ; 350^{\circ} / 82^{\circ} \text {. Author } \\
\text { believes it can be assumed that the fold axis measurements are perpendicular to the movement direction; fault plane at this } \\
\text { point roughly strikes } 090^{\circ} \text { and dip } 75^{\circ} \mathrm{S} \text {; bedding in marble just north of fault, } 035^{\circ} / 17^{\circ} \mathrm{E} \text {. } \\
\text { Fold axis just east of previous readings. Intersection near another fault: } 134^{\circ} / 54^{\circ} ; 133^{\circ} / 31^{\circ} ; 121^{\circ} / 25^{\circ} ; 149^{\circ} / 51^{\circ} ; 99^{\circ} / 67^{\circ} \text {. } \\
\text { Fault continues east at a bearing of } 104^{\circ} \text {; bearing of small fault north of this location, } 134^{\circ} \text {. }\end{array}$} \\
\hline $\mathrm{K}-26$ & & & 6 & 417226 & 6855110 & \multicolumn{2}{|c|}{$\begin{array}{l}\text { Elevation: } 2,190 \text { feet } \\
\text { Folded, black, carbonaceous, fissile shale with up to } 10 \text { inches of limonitic concretions; strike and dip readings: } 342^{\circ} / 24^{\circ} \mathrm{E} ; \\
97^{\circ} / 15^{\circ} \mathrm{N} ; 82^{\circ} / 46^{\circ} \mathrm{S} \text {. Chickaloon; outcrop in recent slide cut along road. }\end{array}$} \\
\hline $\mathrm{K}-27$ & & & 6 & 417183 & 6855000 & \multicolumn{2}{|c|}{$\begin{array}{l}\text { Elevation: } 2,120 \text { feet } \\
\text { Good bedding in Chickaloon; dark shale } 120^{\circ} / 35^{\circ} \mathrm{N} \text {. Bedding just to the south along road } 096^{\circ} / 53^{\circ} \mathrm{N} \text {. Section measurement } \\
\text { Location } 5\end{array}$} \\
\hline $\mathrm{K}-28$ & & & 6 & 417184 & 6854944 & \multicolumn{2}{|c|}{$\begin{array}{l}\text { Elevation: } 2,110 \text { feet } \\
\text { Strike and dip } 274^{\circ} / 48^{\circ} \mathrm{N} \text {; Chickaloon sandstone; bedding } 090^{\circ} / 46^{\circ} \mathrm{N} \text { Elevation: } 2,030 \text { feet; section measurement Location } 4\end{array}$} \\
\hline $\mathrm{K}-29$ & $\begin{array}{l}\text { SC-1 } \\
\text { SC-2 } \\
\text { SC-4 }\end{array}$ & & 6 & 417085 & 6854800 & $\begin{array}{l}\text { Elevation: } 2,030 \text { feet } \\
6 / 27 / 77 \\
\text { Chickaloon siltstone. } \\
\text { Bedding } 105^{\circ} / 52^{\circ} \mathrm{N} . \\
\text { Revisited } 7 / 3 / 77 \\
\text { Elevation: } 2,020 \text { feet } \\
\text { Section starts (Location } 1 \text { ) } \\
\text { south from K-29 along } \\
\text { road; Section represents } \\
\text { Chickaloon Formation. } \\
\end{array}$ & 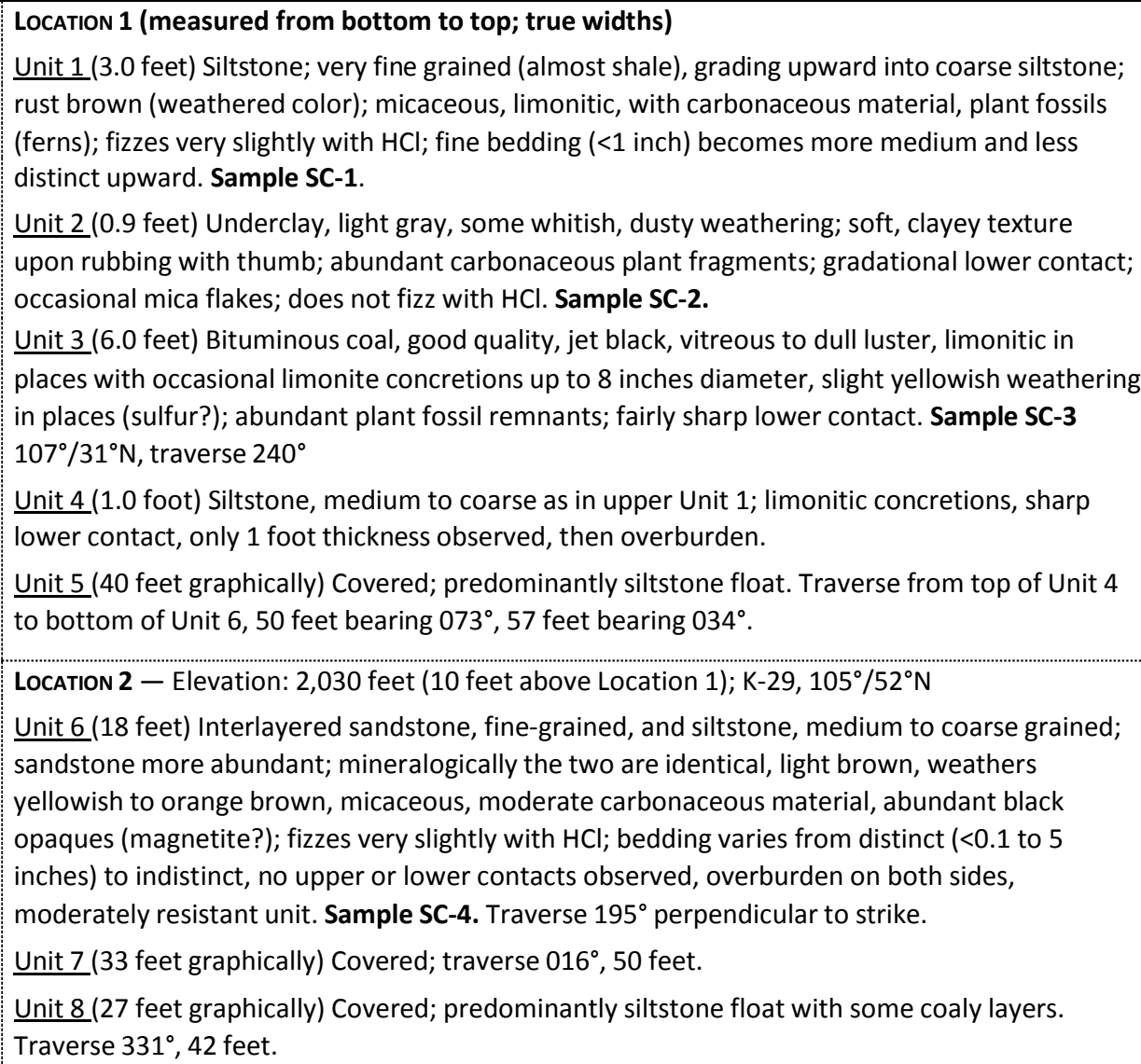 \\
\hline
\end{tabular}


CASTLE MOUNTAIN - CARIBOU FAULT SYSTEM FIELD NOTES (referenced to station localities - UTM NAD27 CONUS)

by William A. Fuchs

\begin{tabular}{|c|c|c|c|c|c|c|c|}
\hline NOTE & SAMPLE & LOCATION & ZN & EASTING & NORTHING & & DESCRIPTION \\
\hline & $\begin{array}{l}\text { SC-5 } \\
\text { SC-6 }\end{array}$ & & & & & (1) & 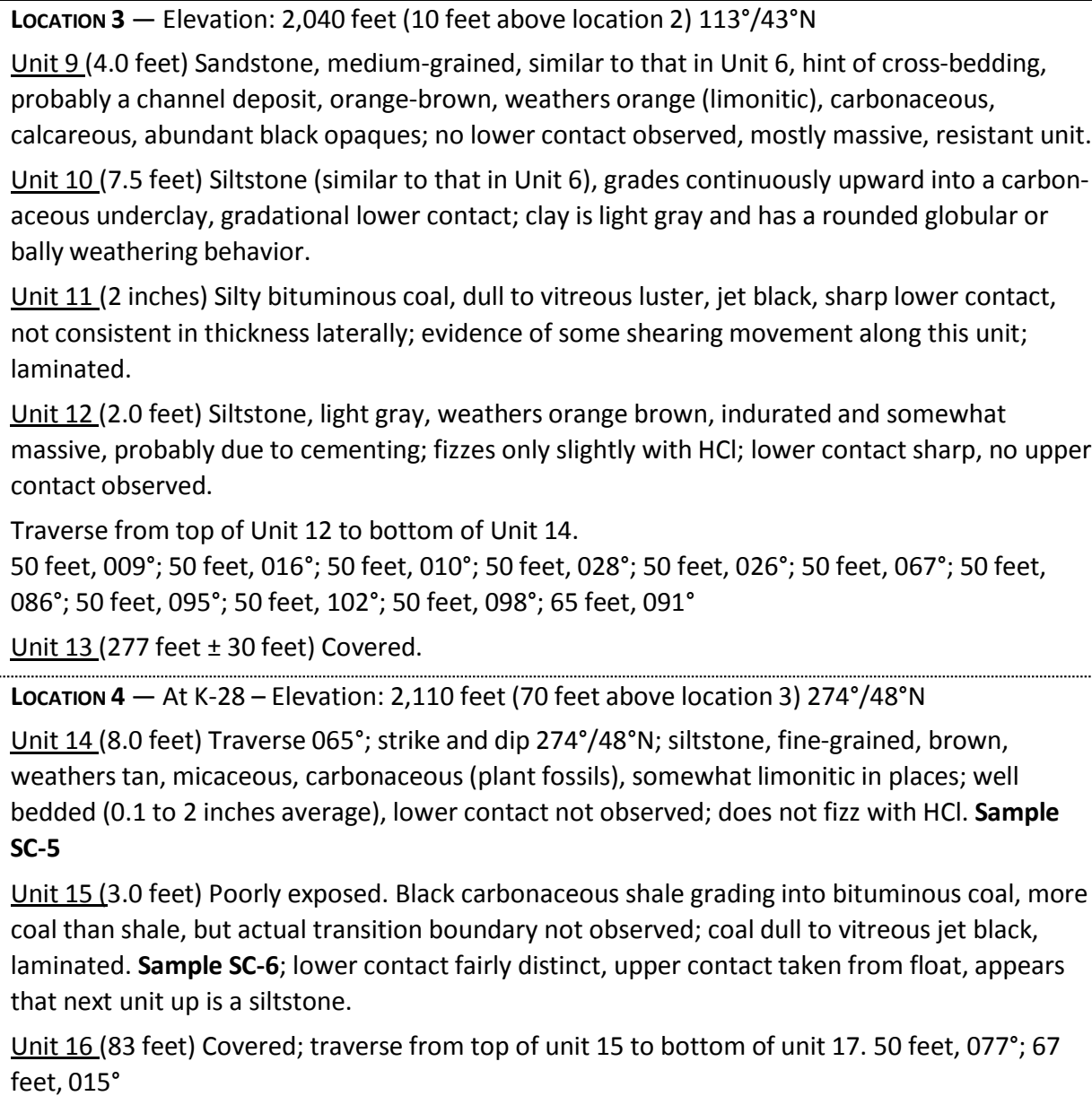 \\
\hline
\end{tabular}


CASTLE MOUNTAIN - CARIBOU FAULT SYSTEM FIELD NOTES (referenced to station localities - UTM NAD27 CONUS)

by William A. Fuchs

\begin{tabular}{|c|c|c|c|c|c|c|c|}
\hline NOTE & SAMPLE & LOCATION & ZN & EASTING & NORTHING & & DESCRIPTION \\
\hline & $\begin{array}{l}\text { SC-7 } \\
\text { SC-8 } \\
\text { SC-9 } \\
\text { SC-10 } \\
\text { SC-11 } \\
\text { SC-12 } \\
\text { SC-13 } \\
\text { SC-14 } \\
\text { SC-15 } \\
\text { SC-16 }\end{array}$ & & & & & (1) & 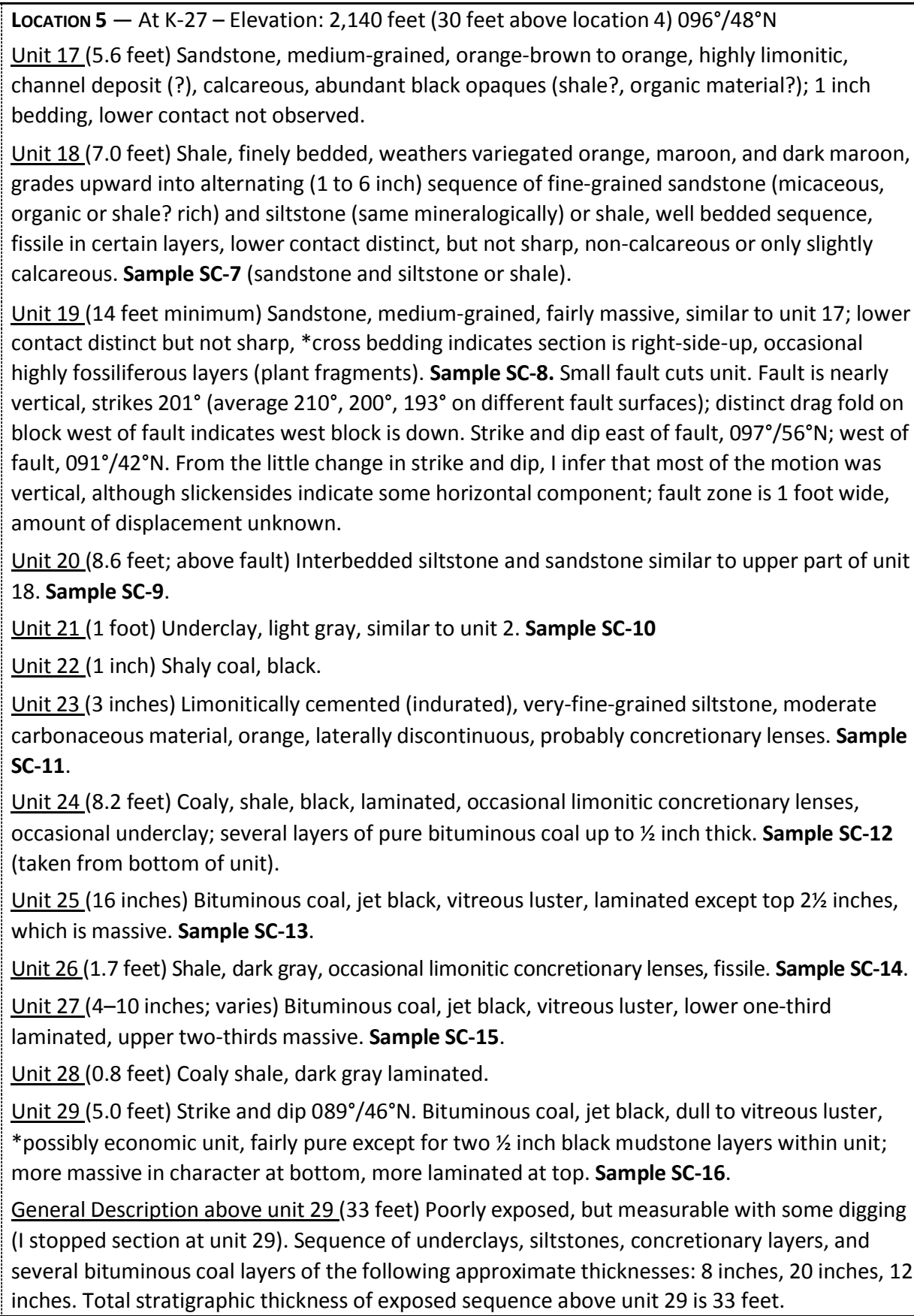 \\
\hline
\end{tabular}


CASTLE MOUNTAIN - CARIBOU FAULT SYSTEM FIELD NOTES (referenced to station localities - UTM NAD27 CONUS)

by William A. Fuchs

\begin{tabular}{|c|c|c|c|c|c|c|}
\hline NOTE & SAMPLE & LOCATION & ZN & EASTING & NORTHING & DESCRIPTION \\
\hline $\mathrm{K}-30$ & & & 6 & 416950 & 6857543 & $\begin{array}{l}\text { Elevation: } 1,840 \text { feet } \\
\text { Prominent fracture in Chickaloon siltstone, } 117^{\circ} / 49^{\circ} \mathrm{S} \text {. Bedding (downslope } 75 \text { feet) } 015^{\circ} / 83^{\circ} \mathrm{E} \text {. }\end{array}$ \\
\hline $\mathrm{K}-31$ & & & 6 & 417052 & 6857539 & $\begin{array}{l}\text { Elevation: } 1,865 \text { feet } \\
\text { Bedding in Chickaloon sandstone } 017^{\circ} / 53^{\circ} \mathrm{E} \text {. }\end{array}$ \\
\hline K-32 & & & 6 & 417104 & 6857599 & $\begin{array}{l}\text { Elevation: } 2,000 \text { feet } \\
\text { In large scar north of camp stream. Bedding in Chickaloon sandstone } 330^{\circ} / 43^{\circ} \mathrm{E} .\end{array}$ \\
\hline $\mathrm{K}-33$ & $S-3$ & & 6 & 417209 & 6857681 & $\begin{array}{l}\text { Elevation: } 2,330 \text { feet } \\
\text { Pebble-cobble conglomerate; Chickaloon Formation; correlates with that found at K- } 11 .{ }^{*} \text { resistant ridge-forming, mappable } \\
\text { unit ( } 90 \text { feet actual thickness; } 100 \text { feet thick in outcrop). Most cobbles no larger than the size of a fist; trend by projection } \\
\text { across camp stream, } 192^{\circ} .{ }^{*} \text { distinctive, uncommon, but not rare siliceous, fine-grained, turquoise-green, probably cupriferous } \\
\text { pebbles (Sample S-3) may help in differentiating this unit from Wishbone Formation. (Non-calcareous) bedding in interbedded } \\
\text { "dirty", micaceous, hornblende-rich(?), non-calcareous sandstone, } 182^{\circ} / 57^{\circ} \mathrm{E} \text {; other pebbles include white and black chert, } \\
\text { silicified volcanics and/or intrusive, silicified siltstone(?). }\end{array}$ \\
\hline K-34 & S-4 & & 6 & 417355 & 6857791 & $\begin{array}{l}\text { Elevation: } 2,530 \text { feet } \\
\text { Bedding in competent, very micaceous sandstone. Sample S-4: } 029^{\circ} / 57^{\circ} \mathrm{E} \text {. Wishbone Formation contact on ridge just east of } \\
\text { K-34. }\end{array}$ \\
\hline $\mathrm{K}-35$ & S-5 & & 6 & 417439 & 6858852 & $\begin{array}{l}\text { Elevation: } 3,510 \text { feet } \\
\text { Sample S-5: Good sample of diorite, medium-grain, quartz }<10 \% \text {, fairly large phenocrysts, feldspar is slightly milky; horn- } \\
\text { blende going to chlorite; color index } 40 \% \text {; prominent fracture } 50 \text { feet east along slope from } \mathrm{K}-35 \text { is } 110^{\circ} / 75^{\circ} \mathrm{N}\end{array}$ \\
\hline $\mathrm{K}-36$ & & & 6 & 417660 & 6858581 & $\begin{array}{l}\text { Elevation (in question): 4,110 feet } \\
\text { Talkeetna Formation; andesite(?) flow, highly fractured. }\end{array}$ \\
\hline $\mathrm{K}-37$ & S-6 & & 6 & 418015 & 6858714 & $\begin{array}{l}\text { Elevation: } 4,530 \text { feet } \\
\text { Well-bedded (fine to medium) limestone with beautiful small fold; no tops observed, no fossils observed. } 75 \text { feet to the } \\
\text { north of } \mathrm{K}-37 \text { syncline the measured fold axis is } 105^{\circ} \text {. Bedding readings going in counter-clockwise circle from south to north: } \\
277^{\circ} / 35^{\circ} \mathrm{N} ; 272^{\circ} / 23^{\circ} \mathrm{N} ; 042^{\circ} / 07^{\circ} \mathrm{N} ; 312^{\circ} / 26^{\circ} \mathrm{S} ; 290^{\circ} / 45^{\circ} \mathrm{S} ; 276^{\circ} / 20^{\circ} \mathrm{S} ; 095^{\circ} / 50^{\circ} \mathrm{S} ; 106^{\circ} / 46^{\circ} \mathrm{S} ; 113^{\circ} / 18^{\circ} \mathrm{N} \text {. Outcrop is } 50-70 \text { feet } \\
\text { in diameter. Sample S-6. Prominent fracture, } 311^{\circ} / 80^{\circ} \mathrm{S} ; 344^{\circ} / 73^{\circ} \mathrm{E} \text { (average). Limestone highly fractured, abundant calcite } \\
\text { veining. Talkeetna Formation. }\end{array}$ \\
\hline $\mathrm{K}-38$ & & & 6 & 418114 & 6858752 & $\begin{array}{l}\text { Elevation: 4,670 feet } \\
\text { Limestone magmatically stoped out by intrusive diorite. Beautiful exposure in gully to north; malachite in quartz vein } 1 \text { foot } \\
\text { wide; magmatic activity probably influenced limestone structure in this area; mainly Talkeetna greenstone south of ridge, } \\
\text { but intrusive goes over to south side just north of K- } 38 \text {. }\end{array}$ \\
\hline $\mathrm{K}-39$ & & & 6 & 418180 & 6858758 & Bedding in limestone, $300^{\circ} / 34^{\circ} \mathrm{S}$ \\
\hline K-40 & & & 6 & 417348 & 6858685 & $\begin{array}{l}\text { Bedding in limestone, } 140^{\circ} / 43^{\circ} \mathrm{E} \text { (probably conforms to general attitude of area). Area contains numerous indistinct, open } \\
\text { folds (wavelength } 12 \text { feet); difficult, but sometimes possible, to distinguish bedding from fracturing. Fold axes (bearing and } \\
\text { plunge) } 145^{\circ} / 24^{\circ} ; 025^{\circ} / 30^{\circ} \text {. }\end{array}$ \\
\hline K-41 & & & 6 & 417792 & 6857157 & $\begin{array}{l}7 / 1 / 77-\text { Elevations are probably } \pm 50 \text { feet } \\
\text { Bedding in Wishbone, pebble-cobble conglomerate (near waterfall) } 225^{\circ} / 18^{\circ} \mathrm{E}\end{array}$ \\
\hline $\mathrm{K}-42$ & & & 6 & 417954 & 6857079 & $\begin{array}{l}\text { Elevation: } 3,490 \text { feet } \\
\text { Bedding in carbonaceous sandstone (stream channel; Wishbone Formation) } 337^{\circ} / 22^{\circ} \mathrm{E} ; 323^{\circ} / 28^{\circ} \mathrm{E} \text { (average } 330^{\circ} / 25^{\circ} \mathrm{E} \text { ) }\end{array}$ \\
\hline K-43 & & & 6 & 418189 & 6856899 & $\begin{array}{l}\text { Elevation: } 3,990 \text { feet } \\
\text { Bedding in pebbly sandstone layer in pebble-cobble conglomerate: } 306^{\circ} / 35^{\circ} \mathrm{E} ; 276^{\circ} / 25^{\circ} \mathrm{E} ; 329^{\circ} / 24^{\circ} \mathrm{E} \text { (average } 304^{\circ} / 28^{\circ} \mathrm{E} \text { ). } \\
\text { Very prominent fractures } \sim 200 \text { feet southeast of } \mathrm{K}-43=003^{\circ} / 73^{\circ} \mathrm{W} \text {, spaced } 3-10 \text { feet apart. }\end{array}$ \\
\hline
\end{tabular}


CASTLE MOUNTAIN - CARIBOU FAULT SYSTEM FIELD NOTES (referenced to station localities - UTM NAD27 CONUS)

by William A. Fuchs

\begin{tabular}{|c|c|c|c|c|c|c|}
\hline NOTE & SAMPLE & LOCATION & ZN & EASTING & NORTHING & DESCRIPTION \\
\hline $\mathrm{K}-44$ & S-7 & & 6 & 418411 & 6856731 & $\begin{array}{l}\text { Elevation: 4,255 feet } \\
\text { Vesicular basalt flow, } 10 \text { feet thick, columnar jointing, fine-grained, light green (chloritic), green phenocrysts (olivine? going } \\
\text { to chlorite) *probably a very good marker horizon in the Wishbone Formation.; appears to thicken ( } 6 \text { feet } \rightarrow 15 \text { feet) in a } \\
\text { southerly direction, suggesting a source in that direction, but absolute direction of thickening unknown; at this point it } \\
\text { appeared that there was a small fault of } 10 \text { feet displacement (south side up) but lack of fracturing in the overlying pebble- } \\
\text { cobble conglomerate and flow pattern, as indicated by vesicles, led me to believe that this represents a depositional surface. } \\
\text { Strike and dips south of stream, } 136^{\circ} / 15^{\circ} \mathrm{E} \text { (upper surface); north of stream, } 127^{\circ} / 12^{\circ} \mathrm{E} \text { (upper surface). Sample S-7 possibly } \\
\text { small synclinal warp with axis going approximately upstream; in streambed basalt is underlain by white tuffaceous pebble } \\
\text { conglomerate ( } 4 \text { feet thick) while south of streambed basalt is underlain by regular Wishbone conglomerate. This further } \\
\text { substantiates depositional surface-versus-fault argument. }\end{array}$ \\
\hline $\mathrm{K}-45$ & & & 6 & 418509 & 6856656 & $\begin{array}{l}\text { Elevation: } 4,560 \text { feet } \\
\text { Bedding in sandstone of Wishbone Formation } 055^{\circ} / 04^{\circ} \mathrm{E}\end{array}$ \\
\hline K-46 & & & 6 & 418242 & 6856462 & $\begin{array}{l}\text { Elevation: 4,390 feet } \\
\text { Bedding in Wishbone Formation, horizontal beds. }\end{array}$ \\
\hline $\mathrm{K}-47$ & & & 6 & 417869 & 6856629 & $\begin{array}{l}\text { Elevation: } 3,815 \text { feet } \\
\text { Bedding in sandstone of Wishbone Formation } 354^{\circ} / 06^{\circ} \mathrm{E}\end{array}$ \\
\hline $\mathrm{K}-48$ & & & 6 & 417649 & 6856865 & $\begin{array}{l}\text { Elevation: } 3,380 \text { feet } \\
\text { Bedding in Wishbone sandstone } 165^{\circ} / 15^{\circ} \mathrm{E}\end{array}$ \\
\hline K-49 & & & 6 & 417684 & 6857474 & $\begin{array}{l}\text { Elevation: } 2,465 \text { feet } \\
\text { Bedding in Wishbone sandstone } 259^{\circ} / 32^{\circ} \mathrm{N}\end{array}$ \\
\hline $\mathrm{K}-50$ & & & 6 & 417635 & 6857559 & $\begin{array}{l}\text { Elevation: } 2,330 \text { feet } \\
\text { Bedding in Wishbone sandstone } 280^{\circ} / 30^{\circ} \mathrm{N}\end{array}$ \\
\hline $\mathrm{K}-51$ & & & 6 & 418769 & 6858123 & $\begin{array}{l}\text { Elevation: } 3,185 \text { feet } \\
\text { Bedding in Wishbone, sandy pebble layer } 016^{\circ} / 32^{\circ} \mathrm{E} \text {; located along permanent stream of topo map }\end{array}$ \\
\hline $\mathrm{K}-52$ & & & 6 & 418885 & 6858310 & $\begin{array}{l}\text { Elevation: } 3,460 \text { feet } \\
\text { Bedding in sandstone unit of Wishbone Formation } 117^{\circ} / 53^{\circ} \mathrm{N}\end{array}$ \\
\hline $\mathrm{K}-53$ & $\mathrm{~T}-1$ & & 6 & 418899 & 6858425 & $\begin{array}{l}\text { Elevation: } 3,580 \text { feet } \\
3 \text { foot fault zone extends to both sides of the canyon; strike and dip } 231^{\circ} / 80^{\circ} \mathrm{N} \text { varies to vertical. This may just be a sedimentary } \\
\text { sandstone to micaceous siltstone; however, I interpret it tentatively as a cataclastic rock. To be confirmed by thin section. } \\
\text { Two Samples: T-1 ( }{ }^{*} \text { newly interpreted as sedimentary unit) }\end{array}$ \\
\hline K-54 & & & 6 & 418788 & 6858624 & $\begin{array}{l}\text { Total width of zone }=\text { previously calculated thickness }+35 \text { feet }(75+35=110 \text { feet) Prominent fractures obviously related to } \\
\text { fault: } 116^{\circ} / 55^{\circ} \mathrm{W}, 320^{\circ} / 37^{\circ} \mathrm{E} \text {. Note, however, that these are fractures in a large block of Wishbone Formation that is within } \\
\text { the fault zone itself and could have been rotated. }\end{array}$ \\
\hline K-55 & & & 6 & 418887 & 6858803 & $\begin{array}{l}\text { Elevation: } 4,410 \text { feet } \\
\text { Bedding on } 3 \text { inch chart bed in Talkeetna limestone } 041^{\circ} / 67^{\circ} \mathrm{E} \text { in place?? Probably the area is highly contorted. Bedding } 50 \\
\text { feet west of } \mathrm{K}-55,005^{\circ} / \text { vertical }\end{array}$ \\
\hline K-56 & & & 6 & 418612 & 6857942 & Bedding in sandstone of Wishbone Formation, $165^{\circ} / 13^{\circ} \mathrm{E}$ \\
\hline
\end{tabular}


CASTLE MOUNTAIN - CARIBOU FAULT SYSTEM FIELD NOTES (referenced to station localities - UTM NAD27 CONUS)

by William A. Fuchs

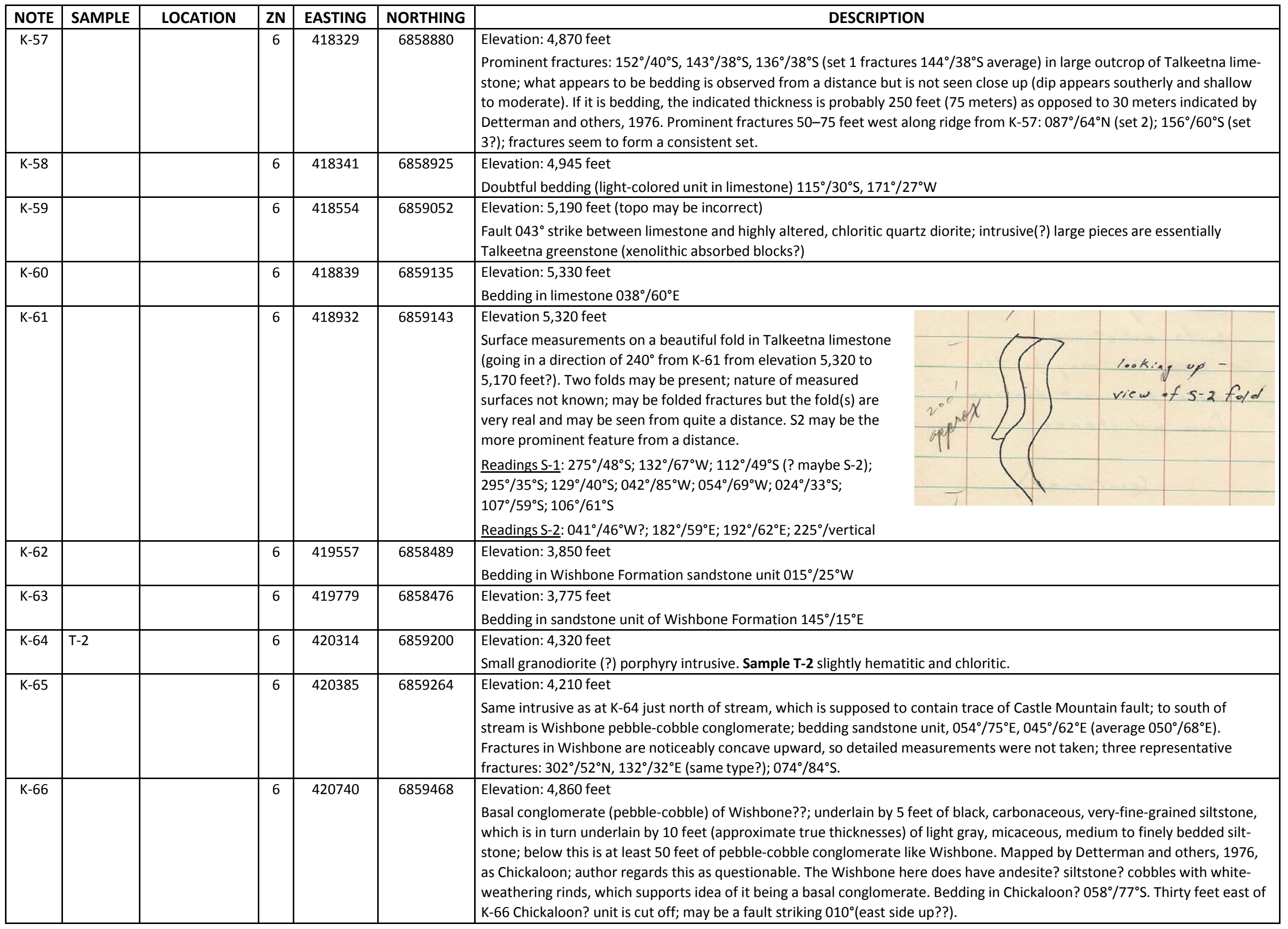


CASTLE MOUNTAIN - CARIBOU FAULT SYSTEM FIELD NOTES (referenced to station localities - UTM NAD27 CONUS)

by William A. Fuchs

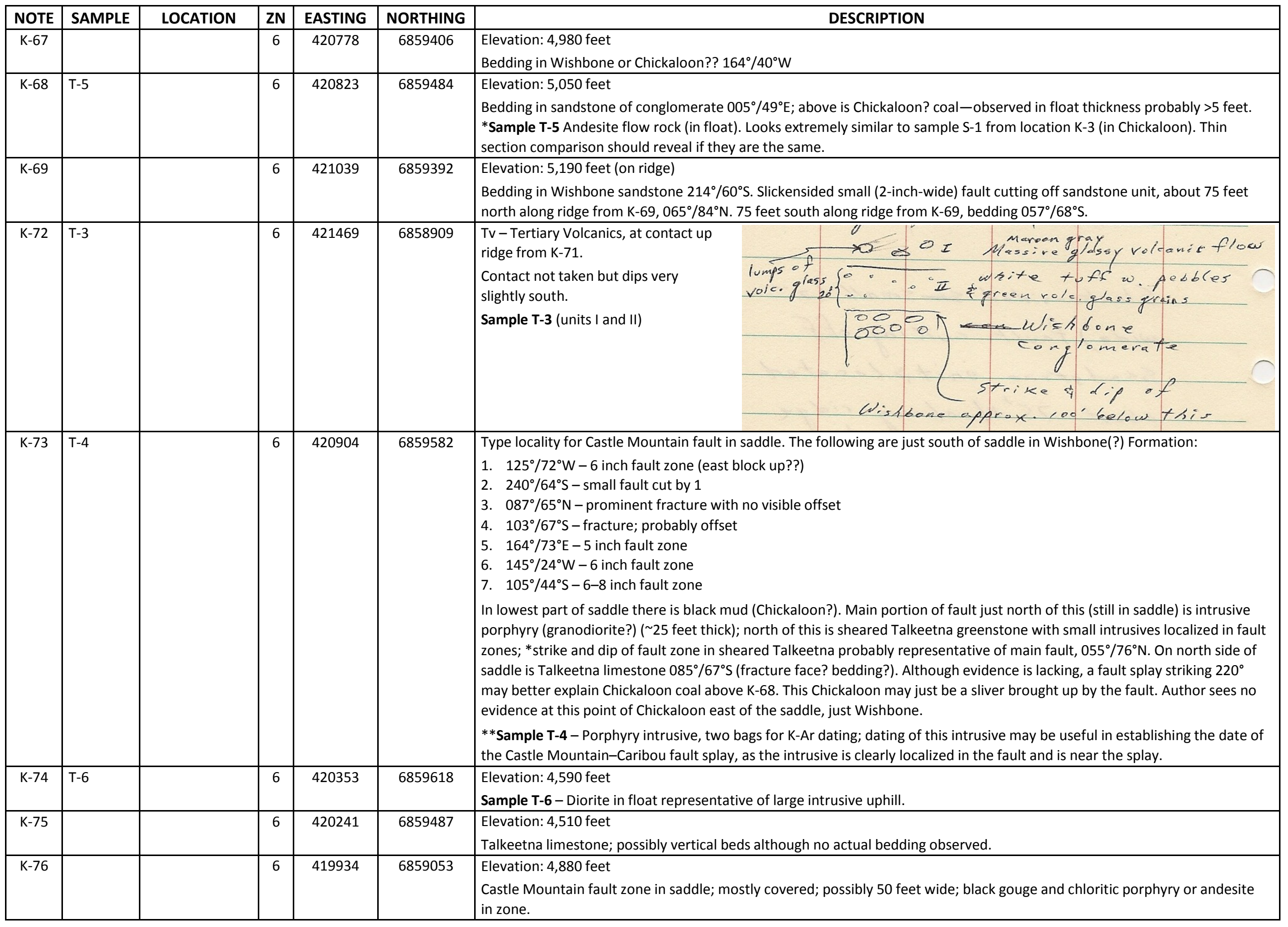


CASTLE MOUNTAIN - CARIBOU FAULT SYSTEM FIELD NOTES (referenced to station localities - UTM NAD27 CONUS)

by William A. Fuchs

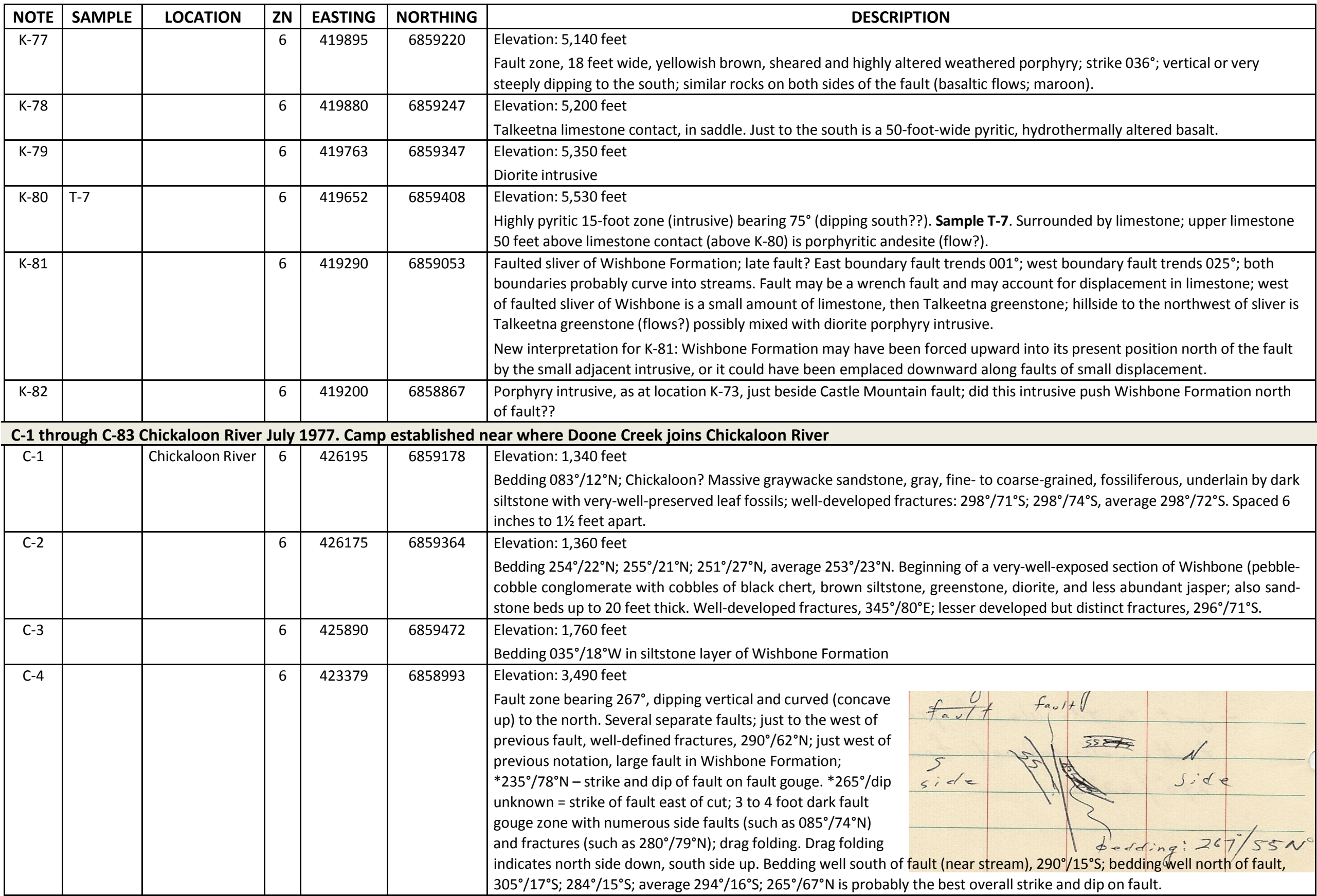


CASTLE MOUNTAIN - CARIBOU FAULT SYSTEM FIELD NOTES (referenced to station localities - UTM NAD27 CONUS)

by William $A$. Fuchs

\begin{tabular}{|c|c|c|c|c|c|c|}
\hline NOTE & SAMPLE & LOCATION & ZN & EASTING & NORTHING & DESCRIPTION \\
\hline$C-5$ & & Doone Creek & 6 & 425151 & 6860018 & $\begin{array}{l}\text { Elevation: } 2,200 \text { feet (not reliable) } \\
\text { Fault, } 247^{\circ} / 78^{\circ} \mathrm{E} \text {; crosses stream; } 1-2 \text { foot dark gouge zone in Wishbone Formation. }\end{array}$ \\
\hline$C-6$ & & & 6 & 425139 & 6860077 & $\begin{array}{l}\text { Bedding in Chickaloon? Formation (siltstone to sandstone sequence, not unlike Matanuska Formation) } 058^{\circ} / \text { vertical; small } \\
\text { fault } 087^{\circ} / 20^{\circ} \mathrm{N} \text {. }\end{array}$ \\
\hline C-7 & $T-8 \mathrm{~A}$ & & 6 & 425266 & 6859951 & $\begin{array}{l}\text { Elevation: } 2,170 \text { feet (not reliable) } \\
\text { West of fault. Bedding in sandstone } 054^{\circ} / 76^{\circ} \mathrm{E} \text {. Fault zone minimum } 20 \text { feet thick; dark gouge with slickensides; abundant } \\
\text { carbonaceous material, concretions, and some coal in fault zone; bedding may generally reflect trend of fault (bedding plane } \\
\text { fault??). East of fault is sequence of pebble conglomerates and sandstones (some siltstones); cross-bedding indicates } \\
\text { sequence is right-side-up; fossils are plant fossils. Rocks are graywackes with abundant muscovite. This may be Chickaloon } \\
\text { Formation instead of Wishbone Formation. Sample T-8A }\end{array}$ \\
\hline C-8 & & & 6 & 424895 & 6860154 & $\begin{array}{l}\text { Elevation: } 2,250 \text { feet } \\
\text { Chickaloon Formation; evidence of contortion and bedding plane faults in coaly layer; in scar north of Doone Creek; location } \\
\text { uncertain from elevation, take location from air photo. }\end{array}$ \\
\hline & & Doone Creek & 6 & & & $\begin{array}{l}\text { 7/27/77 - Beginning of 9-day backpack trip up Doone Creek. Elevation of intersection of side stream near camp at Doone } \\
\text { Creek: 2,560 feet }\end{array}$ \\
\hline C-9 & & & 6 & 424731 & 6860213 & $\begin{array}{l}\text { Elevation: } 2,290 \text { feet } \\
\text { Chickaloon Formation; minor folding due to incompetent coal beds; general strike and dip on sandstone beds } 237^{\circ} / 76^{\circ} \mathrm{N} \\
\text { (north of stream); } 243^{\circ} / 86^{\circ} \mathrm{N} \text { (south of stream); other bedding in folded area } 059^{\circ} / 30^{\circ} \mathrm{N} ; 043^{\circ} / 47^{\circ} \mathrm{N} \text {; prominent fracture with } \\
\text { slickensides } 024^{\circ} / 80^{\circ} \mathrm{E} \text { (pitch on lineation }=30^{\circ} \text { from the south). }\end{array}$ \\
\hline$C-10$ & & & 6 & 424239 & 6860541 & $\begin{array}{l}\text { Elevation: } 2,720 \text { feet (up side creek from camp) } \\
\text { Chickaloon?? Formation; "dirty", fine-grained graywacke sandstone; bedding not observed; very close to fault mapped by } \\
\text { Detterman and others (1976). Highly fractured. Representative fractures: } 125^{\circ} / 84^{\circ} \mathrm{E} \text {, small fault with gouge; } 157^{\circ} / 85^{\circ} \mathrm{E} \text {, frac- } \\
\text { ture; } 141^{\circ} / 79^{\circ} \mathrm{E} \text {, fracture; } 118^{\circ} / 81^{\circ} \mathrm{E} \text {, fracture with slicks; } 150^{\circ} / 64^{\circ} \mathrm{W} \text {, fracture with slicks; } 131^{\circ} / 88^{\circ} \mathrm{W} \text {, fracture; } 114^{\circ} / 87^{\circ} \mathrm{W} \text {, } \\
\text { fracture with slicks. }\end{array}$ \\
\hline C-11 & & & 6 & 424112 & 6860765 & $\begin{array}{l}\text { Elevation: } 2,880 \text { feet } \\
\text { Bedding in Chickaloon Formation; siltstone and coaly shale } 065^{\circ} / \text { vertical }\end{array}$ \\
\hline$C-12$ & & & 6 & 424091 & 6860817 & $\begin{array}{l}\text { Elevation: } 2,910 \text { feet } \\
\text { Cataclasite of large fault zone; zone of most intense faulting is } 80 \text { feet wide (covered to the south); zone of lesser faulting } \\
\text { (but still a cataclasite) is } \sim 100 \text { feet wider to the north; fault trend } 089^{\circ} \text {, dips vertical to steeply south. }\end{array}$ \\
\hline$C-13$ & & & 6 & 424089 & 6860824 & $\begin{array}{l}\text { Elevation: } 2,920 \text { feet } \\
\text { Bedding } 246^{\circ} / 79^{\circ} \mathrm{N} \text {. Talkeetna Formation; contact between green andesite and red andesite, flows? Above C-13 part of an } \\
\text { ammonite } \sim 1 \text { foot in diameter was found. }\end{array}$ \\
\hline C-14 & & & 6 & 424081 & 6860921 & $\begin{array}{l}\text { Elevation: 3,000 feet (located differently on topo for good reasons) } \\
\text { Bedding in well-bedded (beds } 6 \text { inches to } 1 \text { foot thick) Talkeetna andesite flow, } 072^{\circ} / 31^{\circ} \mathrm{N}\end{array}$ \\
\hline C-15 & & & 6 & 424083 & 6860965 & $\begin{array}{l}\text { Elevation: } 3,050 \text { feet } \\
\text { Located at waterfall; farther travel upstream not possible; abundant and well-defined fractures appear to be controlling } \\
\text { stream at this point; significant offset on fractures on west side (zone is } \sim 50 \text { feet across) may even be considered a fault; } \\
\text { fractures: (two prominent sets?) } 342^{\circ} / 83^{\circ} \mathrm{W} ; 009^{\circ} / 89^{\circ} \mathrm{W} ; 137^{\circ} / 74^{\circ} \mathrm{W} ; 147^{\circ} / 82^{\circ} \mathrm{W} ; 153^{\circ} / 70^{\circ} \mathrm{W} ; 337^{\circ} / 88^{\circ} \mathrm{W} ; 333^{\circ} / 76^{\circ} \mathrm{W} \text {. Rock } \\
\text { is Talkeetna Formation, dense, aphanitic to porphyritic, light green andesite, flow?, with white talc-like veining (especially in } \\
\text { fractures). }\end{array}$ \\
\hline C-16 & & & 6 & 424329 & 6860338 & Slump block (of Matanuska Formation?) \\
\hline
\end{tabular}


CASTLE MOUNTAIN - CARIBOU FAULT SYSTEM FIELD NOTES (referenced to station localities - UTM NAD27 CONUS)

by William A. Fuchs

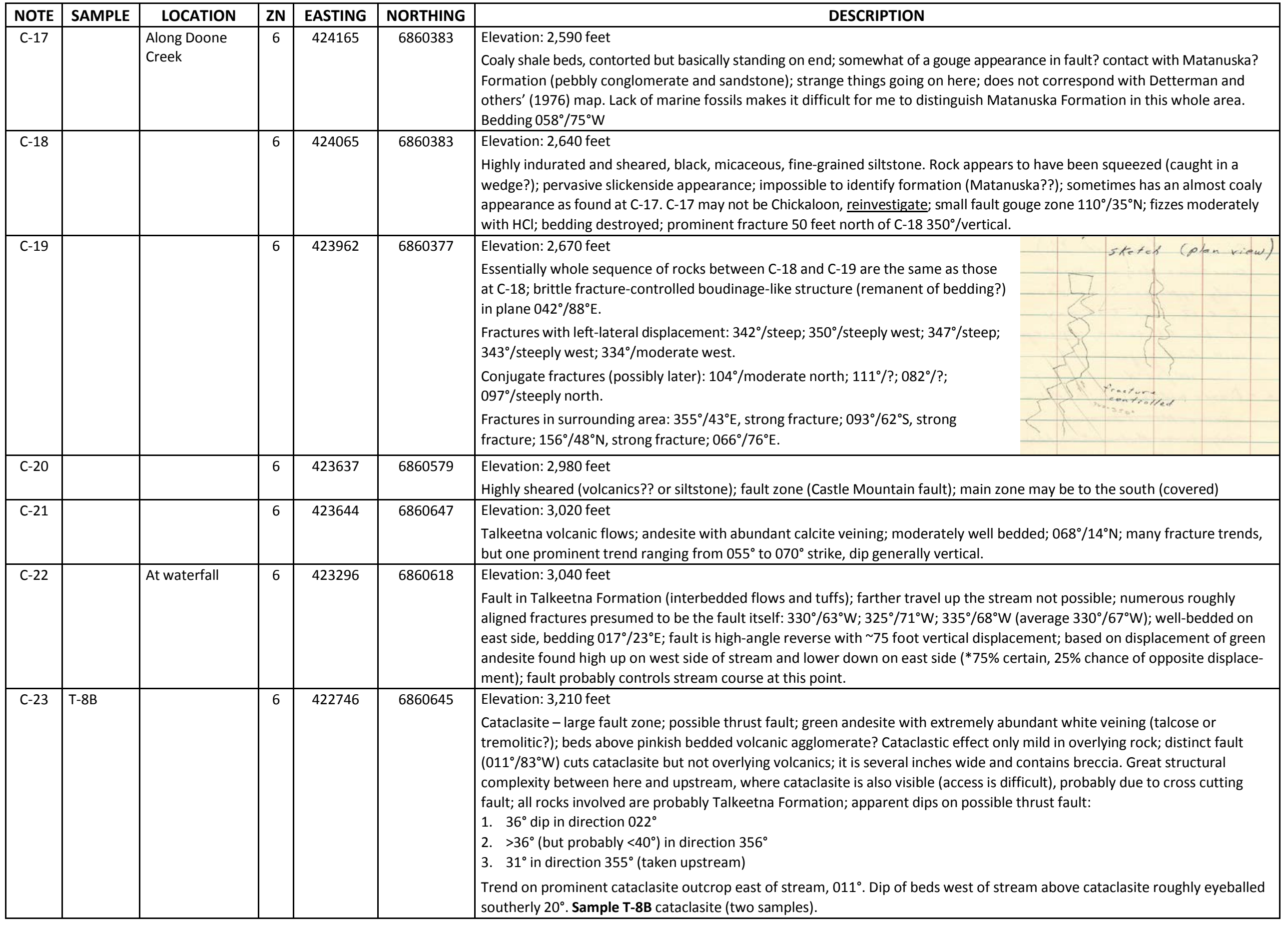


CASTLE MOUNTAIN - CARIBOU FAULT SYSTEM FIELD NOTES (referenced to station localities - UTM NAD27 CONUS)

by William A. Fuchs

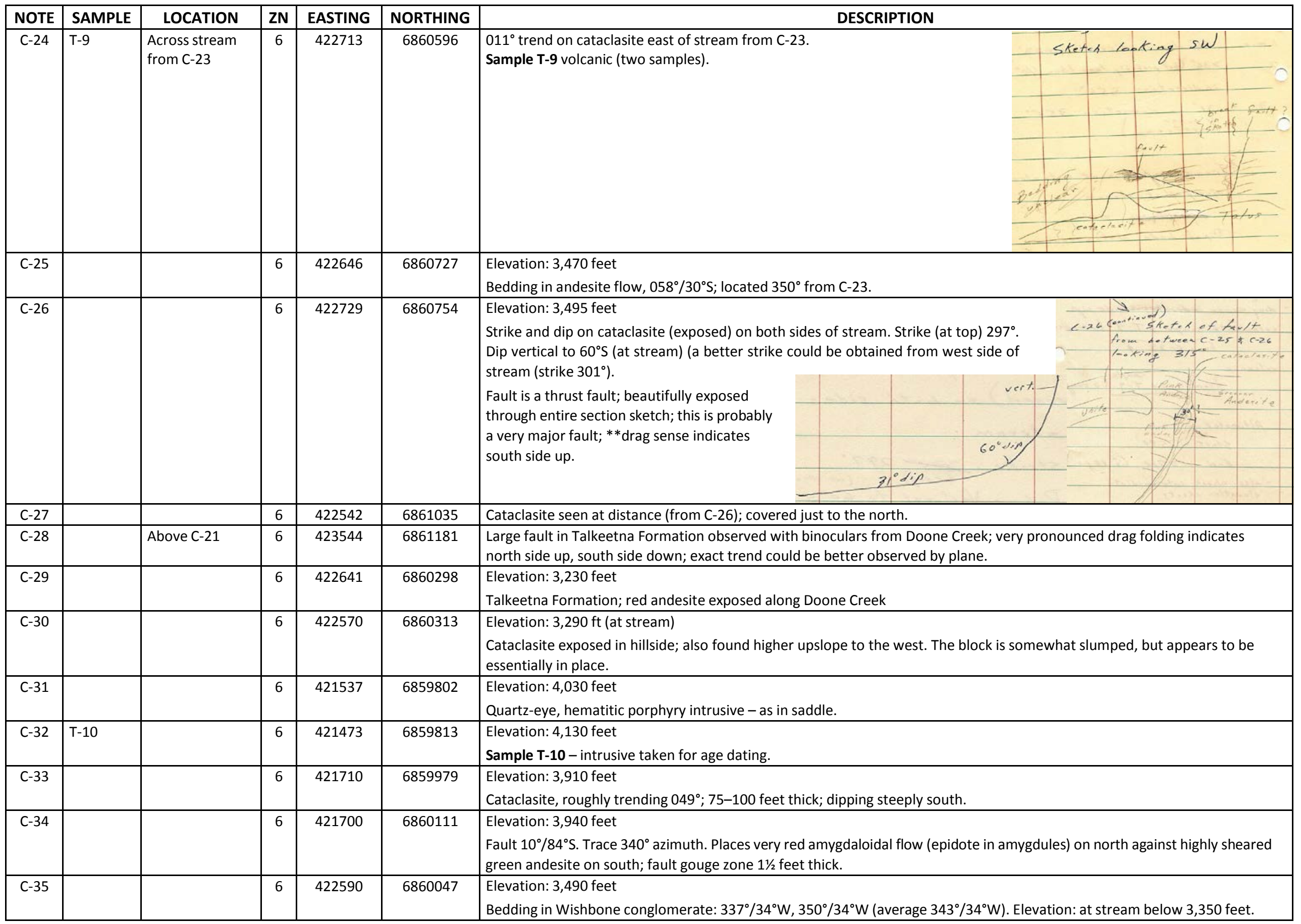


CASTLE MOUNTAIN - CARIBOU FAULT SYSTEM FIELD NOTES (referenced to station localities - UTM NAD27 CONUS)

by William A. Fuchs

\begin{tabular}{|c|c|c|c|c|c|c|}
\hline NOTE & SAMPLE & LOCATION & ZN & EASTING & NORTHING & DESCRIPTION \\
\hline $\mathrm{C}-36$ & & & 6 & 422760 & 6860421 & $\begin{array}{l}\text { Elevation: 3,140 feet } \\
\text { Highly fractured Talkeetna volcanics (no preferred orientation) }\end{array}$ \\
\hline$C-37$ & & & 6 & 422584 & 6860543 & $\begin{array}{l}\text { Elevation: } 3,280 \text { feet } \\
\text { Bedding in Talkeetna purple agglomerate } 213^{\circ} / 26^{\circ} \mathrm{E}\end{array}$ \\
\hline C-38 & & & 6 & 422389 & 6860635 & $\begin{array}{l}\text { Elevation: } 3,420 \text { feet } \\
\text { Large fault zone ( } 85 \text { feet wide); cataclasite and fault breccia exposed in stream cut; just south of zone fault }-249^{\circ} / 20^{\circ} \mathrm{S}-4 \\
\text { inch gouge zone, fault }-248^{\circ} / 64^{\circ} \mathrm{N}-1 \text { foot fault breccia; in zone itself } 215^{\circ} / 56^{\circ} \mathrm{N} \text {; however, other trends are present and } \\
\text { this may not reflect overall trend. High-angle portion of a large thrust fault?? }\end{array}$ \\
\hline$C-39$ & T-11 & & 6 & 422245 & 6860759 & $\begin{array}{l}\text { Elevation: } 3,540 \text { feet } \\
\text { Small fault zone cutting thrust fault, } 106^{\circ} / 60^{\circ} \mathrm{N} \text {; black, dense unit at stream; this unit can be followed downstream on east } \\
\text { side and then on west side for a distance of } 200 \text { feet - highly chewed up - maximum thickness of several feet; good evidence } \\
\text { that I am walking along the plane of a thrust fault. Is this black unit, which is calcareous, the Talkeetna limestone smeared } \\
\text { out along the thrust? Thin section analysis needed. Sample T-11 **All rocks along stream from C-38 to C-39 and beyond are } \\
\text { highly faulted, cataclastic, and hydrothermally altered in places. If this is a thrust, it is huge and the fault plane itself has a } \\
\text { minimum thickness in places of } 100 \text { feet. I believe at this point that this fault correlates with the one found in the canyon } \\
\text { just to the west. }\end{array}$ \\
\hline$C-40$ & & & 6 & 422178 & 6860820 & $\begin{array}{l}\text { Elevation: } 3,570 \text { feet } \\
\text { Vein } 1-6 \text { feet thick; } 058^{\circ} / 80^{\circ} \mathrm{S} \text {; zone up to } 1 \text { foot thick of } 50 \% \text { pyrite in the middle; crosses stream. }\end{array}$ \\
\hline C-41 & & & 6 & 422020 & 6860940 & $\begin{array}{l}\text { Elevation: } 3,690 \text { feet } \\
\text { Fault zone } 192^{\circ} / 77^{\circ} \mathrm{E} ; 20 \text { feet thick. Is thrust becoming high angle at this point?? }\end{array}$ \\
\hline C-42 & T-12 & & 6 & 421961 & 6860995 & $\begin{array}{l}\text { Elevation: 3,770 feet (elevation: high??) } \\
\text { End of fault zone. Sample T-12: lower plate rocks just northwest of C-42 }\end{array}$ \\
\hline C-43 & & & 6 & 421936 & 6861052 & $\begin{array}{l}\text { Chickaloon Formation!! Well-bedded dark shales and siltstones. Bedding } 206^{\circ} / 35^{\circ} \mathrm{W} ; ~ 200 \text { feet northwest of fault; note } \\
\text { sense of drag! }\end{array}$ \\
\hline C-44 & & & 6 & 421874 & 6861062 & $\begin{array}{l}\text { Elevation: } 3,850 \text { feet } \\
\text { End of outcrop; siltstone - Chickaloon. Bedding } 241^{\circ} / 25^{\circ} \mathrm{W}\end{array}$ \\
\hline$C-45$ & & & 6 & 421965 & 6861126 & $\begin{array}{l}\text { Elevation: } 4,030 \text { feet } \\
\text { Chickaloon? Formation. Plant fossils. Bedding } 197^{\circ} / 78^{\circ} \mathrm{W} \text {; probably next to fault; prominent fracture with slick } 082^{\circ} / 77^{\circ} \mathrm{N} \text {; } \\
\text { lineation on slickenside } 35^{\circ} \text { from west. }\end{array}$ \\
\hline C-46 & & & 6 & 422554 & 6861278 & $\begin{array}{l}\text { Elevation: } 3,860 \text { feet } \\
\text { Cataclasite (another imbrication on thrust fault?) high angle at this point? More cataclasite continues upstream at least } 500 \\
\text { feet along stream (still another imbricate?) }\end{array}$ \\
\hline C-47 & & & 6 & 422543 & 6861076 & $\begin{array}{l}\text { Elevation: 2,690 feet } \\
\text { Highly fractured, faulted, and altered (not cataclasite, however) volcanics? (andesite flow?) } \\
\text { * Note rocks north of fault described at C-26 are green andesite agglomerates and flows - very fractured. }\end{array}$ \\
\hline C-48 & & & 6 & 423802 & 6860287 & $\begin{array}{l}\text { Elevation: 2,850 feet } \\
\text { Chickaloon Formation; coarse, micaceous sandstone, pebbly in places, plant fossils; no evidence of Matanuska up this canyon } \\
\text { as mapped by Detterman and others (1976). }\end{array}$ \\
\hline C-49 & & & 6 & 423727 & 6860190 & $\begin{array}{l}\text { Elevation: } 3,020 \text { feet } \\
\text { Bedding } 082^{\circ} / 59^{\circ} \text { S; good exposure of well-bedded shale, siltstone, and sandstone (Chickaloon) }\end{array}$ \\
\hline
\end{tabular}


CASTLE MOUNTAIN - CARIBOU FAULT SYSTEM FIELD NOTES (referenced to station localities - UTM NAD27 CONUS)

by William $A$. Fuchs

\begin{tabular}{|c|c|c|c|c|c|c|c|c|c|c|}
\hline NOTE & SAMPLE & LOCATION & ZN & EASTING & NORTHING & \multicolumn{5}{|c|}{ DESCRIPTION } \\
\hline C-50 & & & 6 & 423695 & 6860052 & \multicolumn{5}{|c|}{$\begin{array}{l}\text { Elevation: } 3,290 \text { feet } \\
\text { Bedding } 029^{\circ} / 32^{\circ} \mathrm{E} \text {; Chickaloon black shale; some coal present in section; the decrease in dip upstream substantiates the } \\
\text { presence of a large fault below C- } 49 \text {, if it is assumed to be caused by drag; note that from C- } 49 \text { upstream through C-50 is a } \\
\text { good continuous section, which would be measureable (although with some difficulty); very little structural complication. }\end{array}$} \\
\hline C-51 & & & 6 & 423609 & 6859919 & \multicolumn{5}{|c|}{$\begin{array}{l}\text { Elevation: 3,580 feet } \\
\text { Pebble conglomerate; pebbles mainly black chert, white quartz, minor jasper, no Talkeetna. This is probable Wishbone contact } \\
\text { as used by Detterman and others (1976). }\end{array}$} \\
\hline \multirow[t]{8}{*}{ C-52 } & & & 6 & 423560 & 6859790 & Elevation: 3,880 feet & \multicolumn{4}{|c|}{ General description of lower Wishbone Section from top to base } \\
\hline & & & & & & Beginning of thick se- & FORMATION & THICKNESS & & DESCRIPTION \\
\hline & & & & & & quence of pebble-cobble & Wishbone & - & \multicolumn{2}{|c|}{ Pebble-cobble conglomerate } \\
\hline & & & & & & conglomerate (definite & Wishbone & 40 feet & \multicolumn{2}{|c|}{ Interlayered siltstone or shale and coarse sandstone. } \\
\hline & & & & & & Wishbone - contact of & Wishbone & 50 feet & \multicolumn{2}{|c|}{ Black, highly carbonaceous fissile shale with concretions. } \\
\hline & & & & & & Wishbone that I was using & Wishbone & 175 feet & \multicolumn{2}{|c|}{ Sandstone, fine-grained, well-bedded. } \\
\hline & & & & & & $\begin{array}{l}\text { on King River work). Bed- } \\
\text { ding ( } \sim 60 \text { feet below C-52) }\end{array}$ & Wishbone & 30 feet? & \multicolumn{2}{|c|}{$\begin{array}{l}\text { Pebble conglomerate; pebbles predominantly black chert, white } \\
\text { quartz, minor jasper, little or no Talkeetna or siltstone. }\end{array}$} \\
\hline & & & & & & $\begin{array}{l}265^{\circ} / 28^{\circ} \mathrm{S} \text { in highly carbon- } \\
\text { aceous black shale. }\end{array}$ & Chickaloon & & \multicolumn{2}{|c|}{$\begin{array}{l}\text { Interbedded, highly carbonaceous black shale, siltstone, sandstone } \\
\text { (with pebbles occasionally) with abundant plant fossils. }\end{array}$} \\
\hline C-53 & & & 6 & 422769 & 6860259 & \multicolumn{5}{|c|}{$\begin{array}{l}\text { Elevation: 3,290 feet } \\
\text { Chickaloon? In float (probably near to being in place) plant fossils (but are they from Wishbone above); rock somewhat } \\
\text { chewed up; no compelling reason to call this Matanuska Formation. This makes me have doubts as to the existence of a } \\
\text { splay in this location. }\end{array}$} \\
\hline C-54 & & & 6 & 424367 & 6860378 & \multicolumn{5}{|c|}{$\begin{array}{l}\text { Bedding } 250^{\circ} / 59^{\circ} \mathrm{N} \text {. Chickaloon Formation - abundant plant fossils in dark shale, sandstone, and conglomerate; mapped by } \\
\text { Detterman and others (1976) as Matanuska Formation. }\end{array}$} \\
\hline C-55 & & & 6 & 424948 & 6860892 & \multirow{2}{*}{\multicolumn{5}{|c|}{$\begin{array}{l}\text { Cataclastic Talkeetna Formation; probably part of the Caribou fault zone, the main part of which is probably in the covered } \\
\text { area immediately to the south of this point. } \\
\text { Pebble conglomerate, pebble-cobble conglomerate in places; possibly upper Chickaloon Formation; probably lower Wishbone } \\
\text { Formation; minor carbonaceous material found on close inspection; not Matanuska! Bedding } 064^{\circ} / 65^{\circ} \mathrm{S} \text {. Fractures: } 338^{\circ} / 77^{\circ} \mathrm{E}, \\
006^{\circ} / 30^{\circ} \mathrm{S} \text { - with slickensides, } 186^{\circ} / 67^{\circ} \mathrm{W} \text {. }\end{array}$}} \\
\hline C-56 & & & 6 & 425328 & 6860712 & & & & & \\
\hline$C-57$ & & & 6 & 424497 & 6860274 & \multicolumn{5}{|c|}{$\begin{array}{l}\text { Fault - Castle Mountain segment? } \\
\text { Tentatively I accept the rock exposed in scar north of Doone Creek from C-57 } \\
\text { to C-58 as Chickaloon Formation; dark siltstone (micaceous) with carbonaceous } \\
\text { smears, sandstone, local conglomerate. However, good plant fossils as normally } \\
\text { found in Chickaloon are absent; fault zone observed in scar uphill and east of } \\
\text { C-57 is distinguished by folding in Chickaloon Formation, which is on south side } \\
\text { of fault. }\end{array}$} \\
\hline$C-58$ & & & 6 & 424638 & 6860242 & \multicolumn{5}{|c|}{$\begin{array}{l}\text { Elevation: 2,390 feet (take as plotted) } \\
\text { No evidence that this is a fault contact; very concordant transition; not investigated closely, but Chickaloon Formation (north } \\
\text { of C-58 is taken as the white band that cuts across scar); from float it appears to be conglomerate with plant fossils (including } \\
\text { a tree fragment - which was coal). }\end{array}$} \\
\hline
\end{tabular}


CASTLE MOUNTAIN - CARIBOU FAULT SYSTEM FIELD NOTES (referenced to station localities - UTM NAD27 CONUS)

by William A. Fuchs

\begin{tabular}{|c|c|c|c|c|c|c|}
\hline NOTE & SAMPLE & LOCATION & ZN & EASTING & NORTHING & DESCRIPTION \\
\hline C-59 & & $\begin{array}{l}\text { Near Chickaloon } \\
\text { River }\end{array}$ & 6 & 426903 & 6859280 & $\begin{array}{l}\text { Wishbone Formation - large outcrop. Pebble-cobble conglomerate with abundant sandstone lenses. Bedding: } 160^{\circ} / 10^{\circ} \mathrm{E} \text {, } \\
172^{\circ} / 14^{\circ} \mathrm{E}, 202^{\circ} / 11^{\circ} \mathrm{E}, 150^{\circ} / 20^{\circ} \mathrm{N} \text { (average } 171^{\circ} / 14^{\circ} \mathrm{E} \text { ). Fracture zone } 322^{\circ} / 64^{\circ} \mathrm{S} \text {. Fracture } 287^{\circ} / 15^{\circ} \mathrm{N} \text {. Possibly Chickaloon- } \\
\text { Wishbone; contact is exposed across river. }\end{array}$ \\
\hline$C-60$ & & At waterfall & 6 & 426785 & 6861158 & $\begin{array}{l}\text { Elevation: } 1,540 \text { feet } \\
\text { Chickaloon Formation; interbedded sandstone and pebble conglomerate. Bedding: } 067^{\circ} / 70^{\circ} \mathrm{S}, 059^{\circ} / 79^{\circ} \mathrm{S} \text { (average } 063^{\circ} / 75^{\circ} \mathrm{S} \text { ). } \\
\text { Prominent fractures: } 349^{\circ} / 56^{\circ} \mathrm{N}, 352^{\circ} / 45^{\circ} \mathrm{N} \text { (same set?); } 319^{\circ} / 21^{\circ} \mathrm{N} \text {. Sedimentary features indicate to me that sequence is } \\
\text { right-side-up, but it is not positively certain. }\end{array}$ \\
\hline C-61 & & & 6 & 426619 & 6861239 & $\begin{array}{l}\text { Elevation: } 1,700 \text { feet } \\
\text { Chickaloon Formation; sequence contains equal amounts of pebble conglomerate (some cobbles) and dark siltstone; prom- } \\
\text { inent fractures (south of stream in conglomerate) filled with calcite averaging } 335^{\circ} \pm 3^{\circ} \text { strike; dips steeply east and west. } \\
\text { Conglomerate contains carbonaceous material; siltstone contains some carbonaceous smears and fragments of plant fossils. } \\
\text { Fault (bedding plane fault?) just north of stream } 065^{\circ} / 85^{\circ} \mathrm{E} \text {. }\end{array}$ \\
\hline$C-62$ & & $\begin{array}{l}150 \text { feet up- } \\
\text { stream of C-61 }\end{array}$ & 6 & 426583 & 6861268 & $\begin{array}{l}\text { Elevation: } 1,710 \text { feet } \\
\text { Bedding } 057^{\circ} / 88^{\circ} \mathrm{W} \text {. Chickaloon Formation - dark shale }\end{array}$ \\
\hline C-63 & & & 6 & 426541 & 6861300 & $\begin{array}{l}\text { Elevation: } 1,760 \text { feet } \\
\text { Chickaloon; coaly shale against pebble conglomerate; looks very similar to sequence found at C-7. Is sequence from C-61 to } \\
\text { C- } 63 \text { Wishbone Formation or Matanuska Formation?? (There is a lot of conglomerate in the sequence, but it is more pebble } \\
\text { conglomerate than pebble-cobble conglomerate). Bedding } 064^{\circ} / 88^{\circ} \mathrm{W} \text {. }\end{array}$ \\
\hline$C-64$ & & & 6 & 426445 & 6861313 & $\begin{array}{l}\text { Elevation: } 1,810 \text { feet } \\
\text { Bedding } 030^{\circ} \text { /vertical. Chickaloon Formation; minor open folding overturned to east? Good coal bed. }\end{array}$ \\
\hline C-65 & & $\begin{array}{l}150 \text { feet } \\
\text { downstream }\end{array}$ & 6 & 426207 & 6861280 & $\begin{array}{l}\text { Elevation: } 1,870 \text { feet } \\
\text { End of Chickaloon in float; unit Qal upstream on north side; interval from C-64 to C-65 in very coaly upper Chickaloon?? } \\
\text { Indicates formation downstream probably Wishbone, not Matanuska Formation. }\end{array}$ \\
\hline C-66 & & & 6 & 426034 & 6861320 & $\begin{array}{l}\text { Elevation: } 1,930 \text { feet } \\
\text { Fault rock in float; probably very near to in-place. }\end{array}$ \\
\hline$C-67$ & & & 6 & 426012 & 6861348 & $\begin{array}{l}\text { Elevation: } 1,950 \text { feet } \\
\text { Brecciated limestone (Talkeetna?) with some volcanic fragments; probably in or just along large fault zone; } 30 \text { feet width, } \\
\text { trending roughly } 055^{\circ} \text {. }\end{array}$ \\
\hline$C-68$ & & $\begin{array}{l}\text { Approximately } \\
150 \text { feet up- } \\
\text { stream from } \\
\text { C-67 }\end{array}$ & 6 & 425975 & 6861415 & $\begin{array}{l}\text { Elevation: } 1,980 \text { feet } \\
\text { Very well exposed fault zone on north side of stream; } 7 \text { feet wide; cuts off red volcanic unit on west (same as upper plate } \\
\text { thrust rocks up Doone Creek. Gouge on east and west boundaries of fault, cataclasite (rock type similar to unit above red } \\
\text { volcanics) occupies most of zone; cataclasite (red volcanics) to east. Fault surfaces } 208^{\circ} / 80^{\circ} \mathrm{W} \text { (east boundary), } 210^{\circ} / 85^{\circ} \mathrm{W} \\
\text { (east boundary), } 218^{\circ} / 85^{\circ} \text { (west boundary); drag on west side indicates west side down; fault may cut off brecciated lime- } \\
\text { stone unit to the southwest. Bedding } 058^{\circ} / 37^{\circ} \mathrm{W} \text { (south side of stream); displacement on fault not known but probably large. }\end{array}$ \\
\hline C-69 & & & 6 & 425949 & 6861461 & $\begin{array}{l}\text { Elevation: } 2,020 \text { feet } \\
\text { Contact of pink andesites with gray and white andesite flows (actual contact } \sim 15 \text { feet lower in elevation) unconformable con- } \\
\text { tact; white and gray volcanics (weathered colors) dip gently northward; further travel upstream possible only by wading. }\end{array}$ \\
\hline C-70 & & & 6 & 426330 & 6861820 & $\begin{array}{l}\text { Elevation: } 2,400 \text { feet } \\
\text { Andesite flow - quite chewed up; small exposure }\end{array}$ \\
\hline C-71 & & & 6 & 426338 & 6861915 & $\begin{array}{l}\text { Elevation: } 2,540 \text { feet } \\
\text { Diorite intrusive in contact with andesite flow (andesite above, diorite below); forms distinct white cliffs; diorite similar to } \\
\text { that found in Kings River area but is cataclastically deformed with abundant white veining. }\end{array}$ \\
\hline
\end{tabular}


CASTLE MOUNTAIN - CARIBOU FAULT SYSTEM FIELD NOTES (referenced to station localities - UTM NAD27 CONUS)

by William A. Fuchs

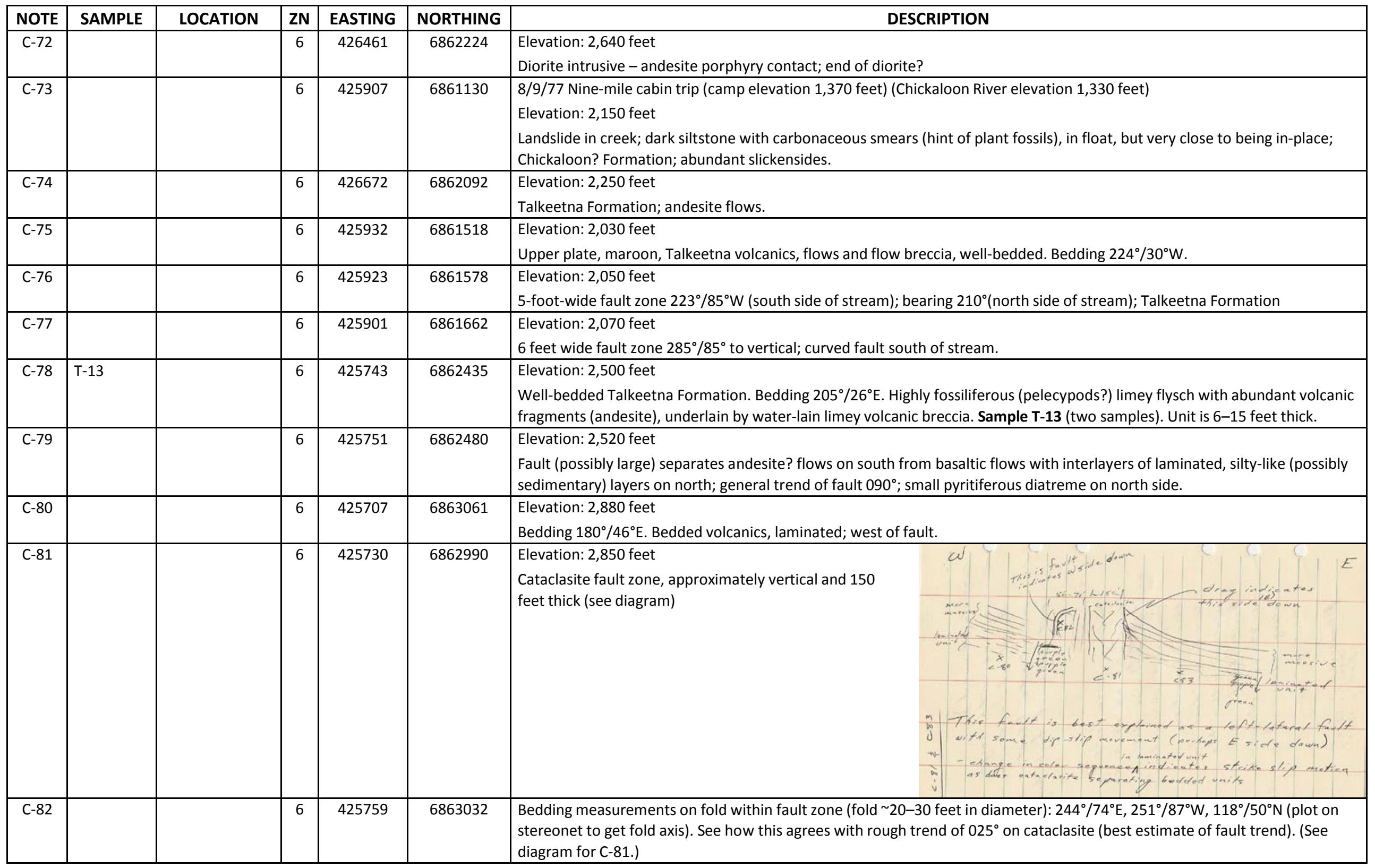


CASTLE MOUNTAIN - CARIBOU FAULT SYSTEM FIELD NOTES (referenced to station localities - UTM NAD27 CONUS)

by William A. Fuchs

\begin{tabular}{|c|c|c|c|c|c|c|}
\hline NOTE & SAMPLE & LOCATION & $\mathrm{ZN}$ & EASTING & NORTHING & DESCRIPTION \\
\hline $\mathrm{C}-83$ & & $\begin{array}{l}150 \text { feet east } \\
\text { along stream } \\
\text { from C-81 }\end{array}$ & 6 & 425742 & 6862967 & $\begin{array}{l}\text { Bedding } 194^{\circ} / 45^{\circ} \text {; same unit as at C- } 80 ;{ }^{*} \text { may be able to determine vertical component of slip from this information; west } \\
\text { side is down from relations within the fault itself. (See diagram for C-81.) } \\
\text { 8/11/77 General Description of Talkeetna Formation - west side Chickaloon River } \\
\text { Two and possibly three large volcanic units are distinguishable. Lower unit is a well-bedded sequence within a maroon color } \\
\text { overall. This unit consists of andesitic flows, more abundant flow breccias, and less abundant agglomerates. I call this maroon } \\
\text { unit 'upper plate volcanics' as it appears as the upper plate of thrusting in the vicinity of the Caribou-Castle Mountain fault } \\
\text { splay. Resting in angular unconformity above this unit is a very thick, well-bedded, andesitic (and possibly basaltic in places) } \\
\text { sequence of whiter-appearing (weathered color) volcanics. In general, this unit has more layers, which are tuffaceous, and } \\
\text { hence the whiter color. A third general volcanic unit is a maroon to green, somewhat cataclastically deformed andesitic vol- } \\
\text { canic (mainly flows and flow breccias). It is not clear whether this actually represents a separate unit or cataclastic deforma- } \\
\text { tion of the other units. It is shot through with white veinlets of calcite and an undetermined talcose-serpentine-like mineral } \\
\text { assemblage. It appears in places that with a little metamorphism it would be the same as the greenstone Talkeetna Forma- } \\
\text { tion in the Kings River area. There is at least one highly fossiliferous (primarily pelecypods?) limey, flysch-like unit with abun- } \\
\text { dant andesite fragments and good bedding. The unit is } 6-15 \text { feet thick. It is not known whether the fossiliferous unit belongs } \\
\text { to the upper plate volcanics or the more tuffaceous unit. }\end{array}$ \\
\hline \multicolumn{7}{|c|}{ 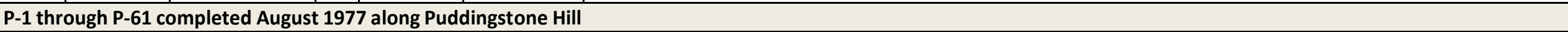 } \\
\hline $\mathrm{P}-1$ & & $\begin{array}{l}\text { Puddingstone } \\
\text { Hill Camp }\end{array}$ & 6 & 428835 & 6859833 & $\begin{array}{l}\text { Elevation: 2,830 feet } \\
\text { Dark carbonaceous siltstone and graywacke sandstone in float - close to in-place; Chickaloon Formation. }\end{array}$ \\
\hline P-2 & & & 6 & 428879 & 6859783 & $\begin{array}{l}\text { Elevation: 2,970 feet } \\
\text { Wishbone contact (Wishbone upstream). Pebble conglomerate, same as at C-51; } 10 \text { feet thick. }\end{array}$ \\
\hline P-3 & & & 6 & 428919 & 6859740 & $\begin{array}{l}\text { Elevation: } 3,090 \text { feet } \\
\text { Bedding (sandstone in Wishbone conglomerate) } 244^{\circ} / 48^{\circ} \mathrm{S}\end{array}$ \\
\hline $\mathrm{P}-4$ & & & 6 & 428957 & 6859693 & $\begin{array}{l}\text { Elevation: } 3,170 \text { feet } \\
\text { Prominent fractures in yellow-brown, altered, tuffaceous sandstone, with cherty concretions: } 188^{\circ} / 75^{\circ} \mathrm{W}, 182^{\circ} / 79^{\circ} \mathrm{W} \\
\text { (average } 185^{\circ} / 77^{\circ} \mathrm{W} \text { ) }\end{array}$ \\
\hline$P-5$ & SP-1 & & 6 & 428985 & 6859603 & $\begin{array}{l}\text { Elevation: } 3,390 \text { feet } \\
\text { White tuffaceous, pebbly sandstone, } 20 \text { feet thick, same as found across and slightly upstream from C-2. Sample SP-1. } \\
\text { Bedding ( } 50 \text { feet at } 330^{\circ} \text { from } \mathrm{P}-5 \text { ) } 242^{\circ} / 40^{\circ} \mathrm{S} \text {. }\end{array}$ \\
\hline P-6 & & & 6 & 429054 & 6859532 & $\begin{array}{l}\text { Elevation: 3,670 feet } \\
\text { Glassy basalt; actual contact probably at 3,640 feet }\end{array}$ \\
\hline $\mathrm{P}-7$ & & & 6 & 429370 & 6859378 & Volcanics \\
\hline $\mathrm{P}-8$ & & & 6 & 429669 & 6859575 & Volcanic vent? Greenish volcanic breccia surrounded by pink volcanic flow. \\
\hline P-9 & & & 6 & 428987 & 6859450 & $\begin{array}{l}\text { Elevation: 3,620 feet } \\
\text { Wishbone-Volcanics contact (actual contact covered) }\end{array}$ \\
\hline P-10 & & & 6 & 428802 & 6859070 & $\begin{array}{l}\text { Elevation: } 3,460 \text { feet (at top of knob) } \\
\text { Volcanics-flow breccia. Bedding } 200^{\circ} / 05^{\circ} \mathrm{E} \text {. }\end{array}$ \\
\hline P-11 & & $340^{\circ}$ from $\mathrm{P}-10$ & 6 & 428757 & 6859127 & $\begin{array}{l}\text { Elevation: 3,270 feet } \\
\text { Wishbone - volcanic contact (Wishbone downhill) }\end{array}$ \\
\hline P-12 & & & 6 & 428927 & 6859954 & $\begin{array}{l}\text { Elevation: } 2,760 \text { feet } \\
\text { First pebble conglomerate (thickness } \sim 40 \text { feet). Bedding } 087^{\circ} / 37^{\circ} \mathrm{S} \text {. Prominent fracture with slickensides, } 130^{\circ} / 87^{\circ} \mathrm{E} \text {, } \\
137^{\circ} / 67^{\circ} \mathrm{E} \text { (average } 134^{\circ} / 77^{\circ} \mathrm{E} \text { ). }\end{array}$ \\
\hline
\end{tabular}


CASTLE MOUNTAIN - CARIBOU FAULT SYSTEM FIELD NOTES (referenced to station localities - UTM NAD27 CONUS)

by William A. Fuchs

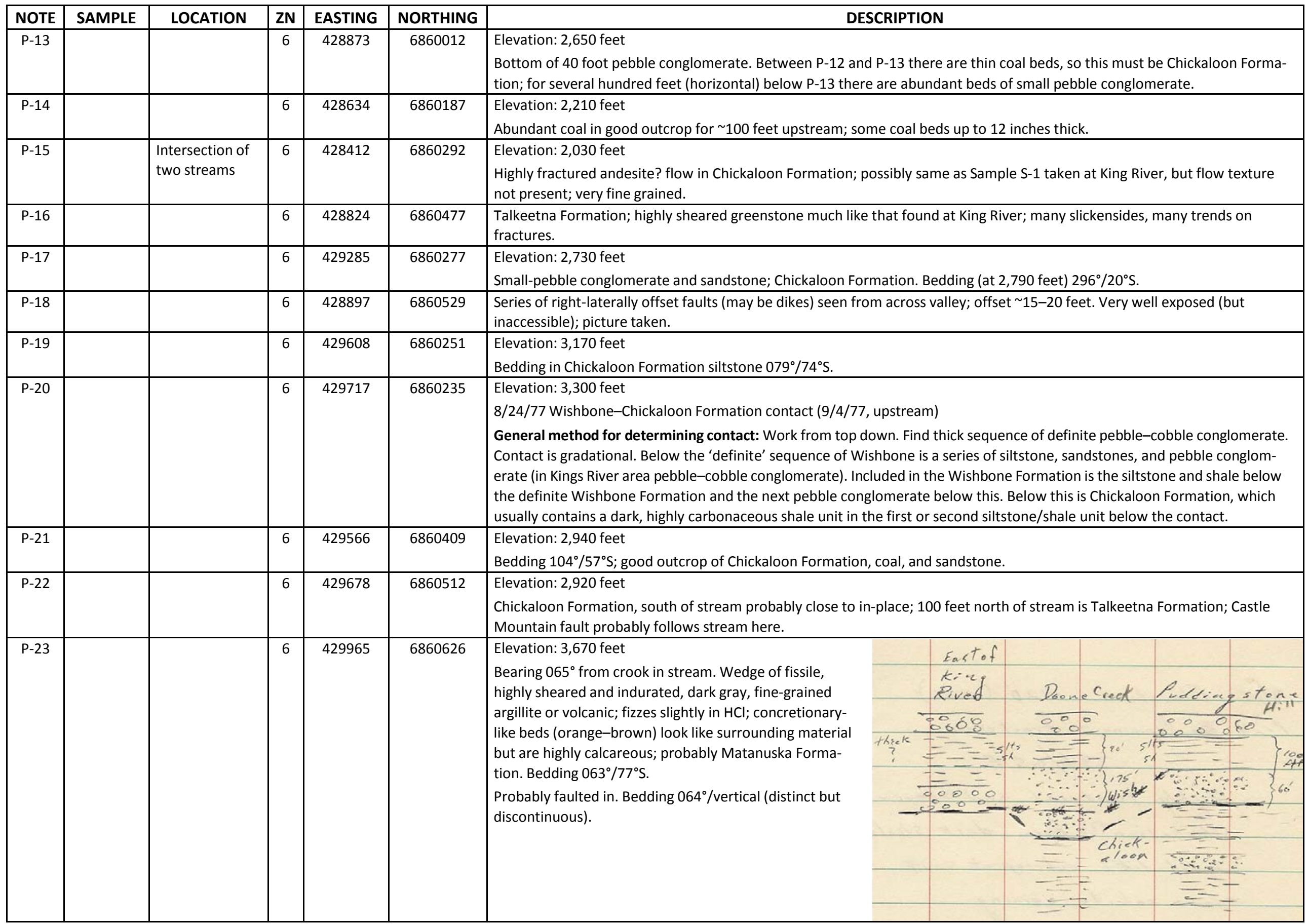


CASTLE MOUNTAIN - CARIBOU FAULT SYSTEM FIELD NOTES (referenced to station localities - UTM NAD27 CONUS)

by William A. Fuchs

\begin{tabular}{|c|c|c|c|c|c|c|}
\hline NOTE & SAMPLE & LOCATION & ZN & EASTING & NORTHING & DESCRIPTION \\
\hline P-24 & & & 6 & 428281 & 6860330 & $\begin{array}{l}\text { Elevation: } 1,920 \text { feet } \\
\text { Prominent fractures } 334^{\circ} / 65^{\circ} \mathrm{E} \text {. Highly fractured and sheared Chickaloon? Formation; dark shale; much like outcrop at C-19; } \\
\text { some calcite veining. (See note for P-28.) }\end{array}$ \\
\hline $\mathrm{P}-25$ & & & 6 & 428120 & 6860237 & $\begin{array}{l}\text { Elevation: } 1,820 \text { feet } \\
\text { Bedding } 282^{\circ} / 77^{\circ} \mathrm{N} \text {; taken on contact between Chickaloon dark shale and andesite flow? (also Chickaloon Formation). } \\
\text { Between P-24 and P-25: highly sheared and indurated Chickaloon shale with andesite flow higher up slope (north side of } \\
\text { stream); complicated and incomprehensible structure. I get the feeling something big is going on here, including folding, } \\
\text { much like what I saw at King River (see K-16 sketch), but the rocks are too messed up to tell. (See note for P-28.) }\end{array}$ \\
\hline P-26 & & & 6 & 428056 & 6860237 & $\begin{array}{l}\text { Elevation: } 1,820 \text { feet } \\
\text { Slickensided fracture next to possible fault gouge } 321^{\circ} / 74^{\circ} \mathrm{N} \text {. Coaly Chickaloon Formation. (See note for P-28.) }\end{array}$ \\
\hline $\mathrm{P}-27$ & & & 6 & 427690 & 6860379 & $\begin{array}{l}\text { Elevation: } 1,640 \text { feet } \\
\text { Bedding in Chickaloon sandstone } 052^{\circ} / 71^{\circ} \text {; less sheared, fractured, and indurated than upstream; coaly section. (See note } \\
\text { for P-28.) }\end{array}$ \\
\hline P-28 & & $\begin{array}{l}\text { Intersection of } \\
\text { stream with } \\
\text { Chickaloon } \\
\text { River }\end{array}$ & 6 & 427226 & 6860784 & $\begin{array}{l}\text { Elevation: 1,340 feet (topo) } \\
* * \text { Note: section from P-24 to P-27 contains andesite flows (or sills) and highly indurated siltstones, which are very difficult to } \\
\text { tell apart. }\end{array}$ \\
\hline $\mathrm{P}-29$ & & $\begin{array}{l}\text { Chickaloon } \\
\text { River }\end{array}$ & 6 & 427095 & 6859674 & $\begin{array}{l}\text { Elevation: } 1,240 \text { feet } \\
\text { Bedding } 264^{\circ} / 47^{\circ} \text { S. Sandstone in good Wishbone conglomerate. }\end{array}$ \\
\hline P-30 & & & 6 & 426862 & 6859213 & $\begin{array}{l}\text { Bedding in Wishbone Formation } 313^{\circ} / 10^{\circ} \mathrm{N} \text { on east side of Chickaloon River; on west side of river I can see siltstone beds, } \\
\text { which are probably lower Wishbone Formation. }\end{array}$ \\
\hline $\mathrm{P}-31$ & & & 6 & 426626 & 6858959 & Dip of bedding $0^{\circ}-2^{\circ}$ (call it horizontal); graywacke sandstone (either lower Wishbone or upper Chickaloon Formation). \\
\hline P-32 & & & 6 & 426670 & 6858737 & $\begin{array}{l}\text { Bedding } 170^{\circ} / 17^{\circ} \mathrm{N} \text {; Chickaloon Formation probably; } 100 \text { foot sequence of siltstone and shale; many plant fossils, abundant } \\
\text { leaves; take Wishbone-Chickaloon contact as being between P-30 and P-31. }\end{array}$ \\
\hline P-33 & & $\begin{array}{l}\text { Bearing } 0761^{\circ} \\
\text { from crook in } \\
\text { stream }\end{array}$ & 6 & 430170 & 6860525 & $\begin{array}{l}\text { Elevation: 3,720 feet } \\
\text { Probable fault contact between Chickaloon Formation on south and Talkeetna Formation on north; fault zone (Castle Moun- } \\
\text { tain fault) is } 250 \text { feet wide here, } 100 \text { feet north of this point and } 150 \text { feet south of this point, fault zone appears to consist } \\
\text { of a swarm of andesite? Dikes ( } 8-10 \text { dikes in all) separated by dark fault gouge and, in places, breccia. Identity of the gouge } \\
\text { is obscure but appears to be somewhat coaly south of P-33 and volcanic north of P-33. } \\
* * \text { The dikes, which are fairly fresh, and the fault gouge are folded. Dating of the dikes, which were probably intruded along } \\
\text { the fault, and a stereonet analysis of the folding might be very illuminating. } \\
\text { Small folded fractures in andesite dike } 35 \text { feet northwest of P- } 33 \text {. Fold axis - bearing } 065^{\circ} \text {, plunge } 20^{\circ} \text {. }\end{array}$ \\
\hline P-34 & & $\begin{array}{l}\text { Bearing } 070^{\circ} \\
\text { from crook in } \\
\text { stream }\end{array}$ & 6 & 430129 & 6860599 & $\begin{array}{l}\text { Elevation: } 3,780 \text { feet } \\
\text { Shear zone, } 1 \text { foot wide, } 102^{\circ} / 88^{\circ} \mathrm{S} \text {; slickensides indicate vertical movement, south side up; in Talkeetna Formation volcanics. }\end{array}$ \\
\hline P-35 & & & 6 & 429933 & 6860718 & $\begin{array}{l}\text { Elevation: } 3,730 \text { feet } \\
\text { Contact } 018^{\circ} / 40^{\circ} \text {; between } 50 \text { feet whitish tuff on top and black highly fractured, aphanitic argillite or volcanic on bottom; } \\
\text { Talkeetna Formation? }\end{array}$ \\
\hline P-36 & & & 6 & 430126 & 6860239 & $\begin{array}{l}\text { Elevation: 3,940 feet } \\
\text { Wishbone-volcanic contact uphill at } \sim 3,970 \text { feet; Wishbone-Chickaloon contact downhill at } \sim 3,850 \text { feet; float from volcanic } \\
\text { basalt or andesite, fine-grained, hornblende crystals occasionally. }\end{array}$ \\
\hline
\end{tabular}


CASTLE MOUNTAIN - CARIBOU FAULT SYSTEM FIELD NOTES (referenced to station localities - UTM NAD27 CONUS)

by William A. Fuchs

\begin{tabular}{|c|c|c|c|c|c|c|}
\hline NOTE & SAMPLE & LOCATION & ZN & EASTING & NORTHING & DESCRIPTION \\
\hline P-37 & & & 6 & 430023 & 6860248 & $\begin{array}{l}\text { Elevation: } 3,800 \text { feet } \\
\text { Basaltic?, very-fine-grained dike, trending } 025^{\circ} \text { (but also intruding in other directions), altered, intruding Chickaloon Forma- } \\
\text { tion, pebble conglomerate, coal } 40 \text { feet uphill. }\end{array}$ \\
\hline$P-38$ & $\mathrm{P}-2$ & & 6 & 430237 & 6860454 & $\begin{array}{l}\text { Elevation: } 3,890 \text { feet (top of fold) } \\
\text { Fold ( } 75 \text { feet across) in Castle Mountain fault zone; folded, light-colored andesite or basalt dikes (up to } 20 \text { feet thick) and } \\
\text { dark fault gouge, often coaly (coked??) in appearance. Sample P-2 (two samples; one for age dating with } \mathrm{K} \text {-Ar; one for thin } \\
\text { section); dating of dike should demonstrate movement on this portion of the splay since the obtained date. Bedding measure- } \\
\text { ments on fold: top of fold } 090^{\circ} / 67^{\circ} \mathrm{S}, 252^{\circ} / 32^{\circ} \mathrm{S}, 103^{\circ} / 58^{\circ} \mathrm{S}, 090^{\circ} / 74^{\circ} \mathrm{S} \text {; inner part of fold } 240^{\circ} / 50^{\circ} \mathrm{S}, 104^{\circ} 86^{\circ} \mathrm{N} \text {; bottom of fold } \\
196^{\circ} / 26^{\circ} \mathrm{E}, 166^{\circ} / 34^{\circ} \mathrm{E}, 112^{\circ} / 49^{\circ} \mathrm{E}, 301^{\circ} / 79^{\circ} \mathrm{E}, 144^{\circ} / 31^{\circ} \mathrm{E} \text {; funny things going on at hinge } 112^{\circ} / 85^{\circ} \mathrm{N}, 087^{\circ} / 88^{\circ} \mathrm{N}, 062^{\circ} / 73^{\circ} \mathrm{S} \text {; } \\
\text { south limb of fold } 065^{\circ} / 64^{\circ} \mathrm{S}, 082^{\circ} / 70^{\circ} \mathrm{S} \text {. (Pictures taken close-up with Miriam looking } 060^{\circ} \text { and far shot looking } 080^{\circ} \text {.) } \\
\text { Reasons I know dikes are folded rather than intruded that way: } \\
\text { 1. Two and possibly three dikes are concordantly folded. } \\
\text { 2. Surrounding Chickaloon Formation gouge is generally concordant with the fold, and where it isn't, it is folded in small } \\
\text { folds, including chevron folds. }\end{array}$ \\
\hline P-39 & $P-3$ & & 6 & 430582 & 6860537 & $\begin{array}{l}\text { Elevation: } 4,660 \text { feet }(8 / 30 / 77 \text { ) } \\
\text { Contact between andesite or basalt flow (with biotite or phlogopite phenocrysts) on south and argillite or possibly volcanics } \\
\text { on north, which has erratic, carbonate-rich (siderite) concretions (Matanuska Formation?, possibly Chickaloon Formation); } \\
\text { probably same as at P-41 up the ridge. Sample P-3 (two samples of argillite) is a wedge between two faults; north fault splay } \\
\text { also defined by andesite dikes. } \\
8 / 31 / 77 \text { - As seen from a distance, unit appears to dip northerly } ~ 30^{\circ} \text {. }\end{array}$ \\
\hline P-40 & & & 6 & 430554 & 6860430 & $\begin{array}{l}\text { Andesite? flow with basalt or andesite dikes same as in fault zone; disregard Tim mapped unit on Detterman and others } \\
\text { (1976) map. }\end{array}$ \\
\hline P-41 & & & 6 & 430656 & 6860682 & $\begin{array}{l}\text { Talkeetna Formation volcanics; some argillite rocks in unit - blocks; near fault; does Talkeetna Formation die here and not } \\
\text { continue eastward? }\end{array}$ \\
\hline P-42 & $\begin{array}{l}\text { P-4 } \\
\text { P-5 }\end{array}$ & & 6 & 430990 & 6861039 & $\begin{array}{l}\text { Elevation: 5,185 feet } \\
\text { Andesite? flow; weathers fissile; Talkeetna Formation; all formerly named argillite is probably volcanic (has limestone con- } \\
\text { cretions - probably subaqueous). Sample P-4 (two samples): Do thin section work; hint of bedding? dipping } 3^{\circ} \text { in easterly } \\
\text { direction. Sample P-5: Sample of biotitic (or phlogopitic) graywacke, sandstone(?) same as described at P-39; indicates north- } \\
\text { on-north fault splay; very distinctive rock; useful marker horizon. P-5 is light-colored unit at top-it dips in an approximately } \\
\text { northerly direction } 10^{\circ}-15^{\circ} \text {. }\end{array}$ \\
\hline P-43 & & & 6 & 430709 & 6861207 & $\begin{array}{l}\text { Elevation: } 4,830 \text { feet } \\
30 \text { feet south of here is an area of moderately tight folding - fold axes generally trend northeast, plunge } 30^{\circ}\end{array}$ \\
\hline $\mathrm{P}-44$ & & & 6 & 430988 & 6861287 & Not visited (difficult to get to). Some kind of faulting going on in here. \\
\hline P-45 & & & 6 & 428455 & 6860912 & $\begin{array}{l}\text { Elevation: 3,000 feet } \\
\text { Talkeetna Formation; very-fine-grained, somewhat fissile argillite. }\end{array}$ \\
\hline P-46 & P-6 & & 6 & 429034 & 6860975 & $\begin{array}{l}\text { Elevation: } 3,750 \text { feet } \\
\text { Talkeetna Formation; Miriam insists this is graywacke. I originally believed this to be andesite flow similar to sample P-5 } \\
\text { (biotitic), but thin section work on Sample P-6 shows this to be graywacke. }\end{array}$ \\
\hline P-47 & P-7 & & 6 & 429297 & 6861060 & $\begin{array}{l}\text { Elevation: 3,970 feet } \\
\text { Ammonite? in float; most calcareous, fossiliferous pieces of rock appear to come from concretions in volcanic trap. Sample } \\
\text { P-7 (fossil). }\end{array}$ \\
\hline
\end{tabular}


CASTLE MOUNTAIN - CARIBOU FAULT SYSTEM FIELD NOTES (referenced to station localities - UTM NAD27 CONUS)

by William A. Fuchs

\begin{tabular}{|c|c|c|c|c|c|c|}
\hline NOTE & SAMPLE & LOCATION & ZN & EASTING & NORTHING & DESCRIPTION \\
\hline $\mathrm{P}-48$ & $\mathrm{P}-8$ & & 6 & 429427 & 6861116 & $\begin{array}{l}\text { Elevation: 4,140 feet } \\
\text { **Sample P-8 Trap - typical of this whole ridge; make thin section to confirm that rock is volcanic. } \\
\text { Note: Pictures taken on the way downslope looking toward P-39 (bearing } 115^{\circ} \text { ) of wedge between fault splay. }\end{array}$ \\
\hline P-49 & $\begin{array}{l}P-9 \\
P-10\end{array}$ & & 6 & 428866 & 6861565 & 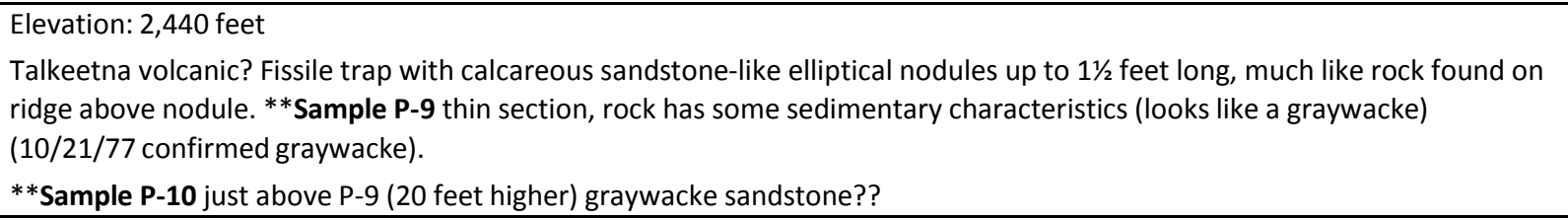 \\
\hline P-50 & & & 6 & 428889 & 6861550 & $\begin{array}{l}\text { Elevation: } 2,490 \text { feet } \\
\text { Bedded sandstone (graywacke like P-10). Bedding } 066^{\circ} / 50^{\circ} \mathrm{W} \text {. }\end{array}$ \\
\hline P-51 & P-11 & & 6 & 428964 & 6861418 & $\begin{array}{l}\text { Elevation: } 2,790 \text { feet } \\
\text { Sedimentary siltstone and sandstone; confirm in thin section with Sample P-11 - calcareous indurate siltstone; no fossils, } \\
\text { but some sedimentary-like flow structure; probably Matanuska - sandstone layers look very much like that up Granite } \\
\text { Creek. Bedding } 073^{\circ} / 61^{\circ} \mathrm{W} \text {. }\end{array}$ \\
\hline $\mathrm{P}-52$ & & & 6 & 429440 & 6861930 & Sandstone as at P-50. \\
\hline $\mathrm{P}-53$ & & & 6 & 428795 & 6862115 & $\begin{array}{l}\text { Elevation: } 1,570 \text { feet } \\
\text { Bedding } 248^{\circ} / 67^{\circ} \mathrm{W} \text {. Matanuska Formation? indurated siltstone. }\end{array}$ \\
\hline $\mathrm{P}-54$ & $\begin{array}{l}P-12 \\
P-13 a \\
P-13 b\end{array}$ & & 6 & 429562 & 6861891 & $\begin{array}{l}\text { Elevation: 2,810 feet } \\
\text { Matanuska Formation? Sample P-12 - Andesite sill or dike? Confirm by thin section. Samples P-13a and P-13b - siltstone } \\
\text { with fossils (Inoceramus? in one). Sample P-12 fizzes slightly in } \mathrm{HCl}-\text { may be sandstone. Bedding } 060^{\circ} / 59^{\circ} \mathrm{W} \text {; highly fossil- } \\
\text { iferous upstream and upslope from P-54 (Inoceramus?). }\end{array}$ \\
\hline P-55 & & & 6 & 429713 & 6861764 & $\begin{array}{l}\text { Elevation: } 3,810 \text { feet } \\
\text { Pebble conglomerate in Matanuska Formation; pebbles up to } 3 \text { inches across, generally elliptical, unit is } 1 \text { foot thick and dis- } \\
\text { continuous laterally; highly indurated. Fault } 297^{\circ} / 72^{\circ} \mathrm{S}, 2 \text { feet wide, contains } 6 \text { inch calcite vein; surrounding rock is biotitic } \\
\text { sandstone, medium grained as in sample P-5; matrix of conglomerate pebble, also biotitic; bedding attitude not certain. }\end{array}$ \\
\hline P-56 & & & 6 & 429844 & 6861762 & $\begin{array}{l}\text { Elevation: 4,060 feet } \\
\text { Medium-grained graywacke sandstone, like sample P-5, biotitic, calcareous cement. }\end{array}$ \\
\hline P-57 & & & 6 & 429776 & 6861730 & $\begin{array}{l}\text { Elevation: } 3,990 \text { feet } \\
\text { Bedding } 220^{\circ} / 34^{\circ} \mathrm{W} \text {. Rock similar to that of P-56. }\end{array}$ \\
\hline $\mathrm{P}-58$ & & & 6 & 429785 & 6862281 & $\begin{array}{l}\text { Elevation: } 2,180 \text { feet } \\
\text { Highly indurated, dark Matanuska Formation siltstone. Bedding } 286^{\circ} / 42^{\circ} \mathrm{N} \text {; prominent fractures } 278^{\circ} / 80^{\circ} \mathrm{S}, 035^{\circ} / 82^{\circ} \mathrm{W} \text {. }\end{array}$ \\
\hline P-59 & & & 6 & 429926 & 6862137 & $\begin{array}{l}\text { Elevation: } 2,580 \text { feet } \\
\text { Diabase dike, ophitic texture, abundant feldspar laths, and pyroxene; contains } 6 \text { inch mixed calcite and diabase vein, minimum } \\
7 \text { feet thick; strike } 114^{\circ} \text {, dip approximately vertical; fairly fine-grained; may be feeder to abundant medium-grained gabbro } \\
\text { float found in stream (pyroxene-rich); surrounding rocks are Matanuska Formation siltstone. }\end{array}$ \\
\hline$P-60$ & & & 6 & 429999 & 6862108 & $\begin{array}{l}\text { Elevation: } 2,770 \text { feet } \\
\text { Pyroxene gabbro intrusive in Matanuska Formation; gabbro is medium- to coarse-grained; much of it is altered to chlorite } \\
\text { and epidote; side canyon does not show up on topo - use air photo mapping. }\end{array}$ \\
\hline P-61 & & & 6 & 430338 & 6862189 & $\begin{array}{l}\text { Elevation: } 3,150 \text { feet } \\
\text { Gabbro intrusive; Matanuska Formation in the float indicates that it is up the hill higher. }\end{array}$ \\
\hline
\end{tabular}


CASTLE MOUNTAIN - CARIBOU FAULT SYSTEM FIELD NOTES (referenced to station localities - UTM NAD27 CONUS)

by William A. Fuchs

\begin{tabular}{|c|c|c|c|c|c|c|}
\hline NOTE & SAMPLE & LOCATION & ZN & EASTING & NORTHING & DESCRIPTION \\
\hline \multicolumn{7}{|c|}{ B-1 through B-191 completed during summer 1978 along Boulder Creek } \\
\hline B-1 & & Boulder Creek & 6 & 439820 & 6865054 & $\begin{array}{l}\text { Elevation: } 3,220 \text { feet } \\
\text { Matanuska Formation; black, indurated siltstone; small fault }-3 \text { inch zone; strike } 001^{\circ} \text {, dip almost vertical, displacement } \\
\text { west side down, } 10 \text { feet(?); many fractures with various displacements. Bedding } 071^{\circ} / 63^{\circ} \mathrm{N} \text {; declination } 262^{\circ} \mathrm{E} \text {. }\end{array}$ \\
\hline B-2 & & & 6 & 439915 & 6865258 & $\begin{array}{l}\text { Elevation: } 3,400 \text { feet } \\
\text { Matanuska Formation. Black siltstones with limestone concretions and biotitic sandstones, abundant Inoceramus. Large fault } \\
\text { strike } 299^{\circ} \text {, high angle, cuts off large siltstone sequence; smaller subsidiary fault strike } 090^{\circ} \text { (average of four readings) with } \\
\text { slickensides; strike-slip motion. }\end{array}$ \\
\hline B-3 & & & 6 & 439739 & 6864580 & $\begin{array}{l}\text { Elevation: } 2,770 \text { feet } \\
\text { Gabbro? Highly weathered, possibly Matanuska sandstone; pyritic in places }\end{array}$ \\
\hline B-4 & & & 6 & 439538 & 6864570 & $\begin{array}{l}\text { Elevation: 3,110 feet } \\
\text { Pebble conglomerate; Matanuska Formation probably (see B-9); pebble of quartzite and andesite?; rock at B-3 may be } \\
\text { Matanuska sandstone. }\end{array}$ \\
\hline B-5 & & & 6 & 439460 & 6864720 & $\begin{array}{l}\text { Elevation: } 3,460 \text { feet } \\
\text { Small fault } 130^{\circ} / 76^{\circ} \mathrm{S}, 11^{1} / 2 \text { foot displacement in Matanuska Formation; argillite with concretion layers, highly chewed up; just } \\
\text { above B-5 (elevation } 3,510 \text { feet): } \\
\text { - Fractures: } 313^{\circ} / 75^{\circ} \mathrm{S} ; 295^{\circ} / 66^{\circ} \mathrm{S} ; 336^{\circ} / 82^{\circ} \mathrm{N} \text {. Bedding } 077^{\circ} / 20^{\circ} \mathrm{N} \text {. } \\
\text { - Fractures (shear fractures): } 107^{\circ} / 62^{\circ} \mathrm{S} ; 296^{\circ} / 57^{\circ} \mathrm{S} ; 48^{\circ} / 61^{\circ} \mathrm{E} \\
\text { Bedding } 241^{\circ} / 37^{\circ} \mathrm{W} \text { (50 feet from previous bedding measurement) } \\
\text { - Bedding } 235^{\circ} / 48^{\circ} \mathrm{W} \text { (just above previous bedding; elevation } 3,620 \text { feet) }\end{array}$ \\
\hline B-6 & & & 6 & 439465 & 6864809 & $\begin{array}{l}\text { Elevation: } 3,660 \text { feet } \\
\text { Gabbro dike, same one as on east side of Boulder Creek probably; appears to be same lithology as gabbro seen last summer } \\
\text { on east side of Chickaloon River. } \\
\text { Elevation: } 3,670 \text { feet } \\
\text { Trend on dike } 312^{\circ} \text {. Cooling joints and/or flow banding: } 317^{\circ} / 88^{\circ} \mathrm{N} ; 320^{\circ} / 87^{\circ} \mathrm{N} ; 320^{\circ} / 90^{\circ} \text {. Cross fractures (joints?) } 049^{\circ} / 74^{\circ} \mathrm{E} \text {; } \\
050^{\circ} / 88^{\circ} \mathrm{E} \text {. }\end{array}$ \\
\hline B-8 & & & 6 & 439536 & 6864424 & $\begin{array}{l}\text { Elevation: } 2,910 \text { feet } \\
\text { Diabase dike along river }\end{array}$ \\
\hline B-9 & & & 6 & 439377 & 6864382 & $\begin{array}{l}\text { Elevation: } 3,110 \text { feet } \\
\text { Bedding in Talkeetna Formation; sandstone } 132^{\circ} / 15^{\circ} \mathrm{S} \text {. This sandstone is underlain by conglomerate such as that at B-4, and } \\
\text { overlain by typical Talkeetna Formation andesite flow. }\end{array}$ \\
\hline B-10 & & & 6 & 439520 & 6864481 & $\begin{array}{l}\text { Elevation: } 3,000 \text { feet } \\
\text { Fault zone } 6 \text { feet wide in Talkeetna Formation. Strike and dip } 210^{\circ} / 90^{\circ} \text {; slickensides indicate left lateral strike-slip movement. }\end{array}$ \\
\hline B-11 & & & 6 & 439454 & 6864557 & $\begin{array}{l}\text { Elevation: } 3,100 \text { feet } \\
\text { Gabbro dike; trend } 282^{\circ} \text {, nearly vertical, } 10 \text { feet thick. Rock above unknown (baked?). }\end{array}$ \\
\hline B-12 & & & 6 & 439287 & 6864553 & $\begin{array}{l}\text { Elevation: } 3,090 \text { feet } \\
\text { Diabase dike; perfect example of sample P-2; } 137^{\circ} / 72^{\circ} \mathrm{S}\end{array}$ \\
\hline B-13 & & & 6 & 439194 & 6864587 & $\begin{array}{l}\text { Elevation: } 3,180 \text { feet } \\
\text { Diabase dike (almost pyroxenite); trend } 105^{\circ} \text {; almost vertical; surrounding rock probably highly baked, impossible to identify } \\
\text { (black) }\end{array}$ \\
\hline
\end{tabular}


CASTLE MOUNTAIN - CARIBOU FAULT SYSTEM FIELD NOTES (referenced to station localities - UTM NAD27 CONUS)

by William $A$. Fuchs

\begin{tabular}{|c|c|c|c|c|c|c|}
\hline NOTE & SAMPLE & LOCATION & ZN & EASTING & NORTHING & DESCRIPTION \\
\hline B-14 & & & 6 & 439004 & 6864378 & $\begin{array}{l}\text { Elevation: } 3,850 \text { feet } \\
\text { Fault between Talkeetna Formation (Horn Mountain member) and Tuxedni??; Matanuska?; 30-foot-wide zone, trend 50, } \\
\text { connects with fault below? }\end{array}$ \\
\hline B-15 & & $\begin{array}{l}\text { Bearing } 267^{\circ} \\
\text { from spot in } \\
\text { river }\end{array}$ & 6 & 438780 & 6864380 & $\begin{array}{l}\text { Elevation: } 4,220 \text { feet } \\
\text { Bedding } 036^{\circ} / 6^{\circ} \mathrm{W} \text {; in Tuxedni? siltstone }\end{array}$ \\
\hline B-16 & & & 6 & 439314 & 6864670 & $\begin{array}{l}\text { Elevation: } 3,230 \text { feet } \\
\text { Bedding } 290^{\circ} / 27^{\circ} \mathrm{N} \text {; Matanuska Formation }\end{array}$ \\
\hline B-17 & & & 6 & 439205 & 6864537 & $\begin{array}{l}\text { Elevation: } 3,230 \text { feet } \\
\text { Bedding } 090^{\circ} / 42^{\circ} \mathrm{N} \text {; Matanuska; sandstone bed }\end{array}$ \\
\hline B-18 & & & 6 & 438743 & 6864921 & Not Listed - No Entry \\
\hline B-19 & & & 6 & 438924 & 6863400 & $\begin{array}{l}\text { Elevation: 3,110 feet } \\
\text { Silicified tuff; Talkeetna Formation }\end{array}$ \\
\hline B-20 & $\begin{array}{l}\text { SB-1 } \\
\text { SB-1-AM }\end{array}$ & & 6 & 438684 & 6863403 & $\begin{array}{l}\text { Elevation: 3,510 feet } \\
\text { Top of "Whitey the tuff"; marker horizon; very white tuff with moderately large, well-formed sanidine crystals, chlorite-rich } \\
\text { (replaced mafics) on top; same unit seen on adjacent ridges and across Boulder Creek; probably 80-100 feet thick; difficult } \\
\text { to tell because attitude is unknown. }\end{array}$ \\
\hline B-21 & & $\begin{array}{l}\text { In Anchorage D- } \\
3 \text { Quad }\end{array}$ & 6 & 440982 & 6866476 & $\begin{array}{l}\text { Elevation: 2,970 feet } \\
\text { Tuxedni Group; tan-gray siltstone, does not fizz with } \mathrm{HCl} \text {, no bedding apparent }\end{array}$ \\
\hline B-22 & & & 6 & 440878 & 6867126 & $\begin{array}{l}\text { Elevation: } 3,320 \text { feet } \\
\text { Bedding } 221^{\circ} / 20^{\circ} \mathrm{E} \text {; Tuxedni; medium-grained, massive sandstone, low-rank graywacke, moderate amount of hematite } \\
\text { (which probably gives the unit its tan-gray color), mafics and/or opaques, feldspar? Walking up creek to this point indicates } \\
\text { that the rocks are tan-gray siltstones and sandstones (which do not fizz with } \mathrm{HCl} \text { ) and lesser massive gray limestones; few } \\
\text { fossils. Plant and bryozoan found; bedding is usually difficult to discern except in this spot. Very little structure except } \\
\text { moderate fracturing. }\end{array}$ \\
\hline B-23 & & & 6 & 440057 & 6867811 & $\begin{array}{l}\text { Elevation: } 4,370 \text { feet } \\
\text { Probably Red Glacier Formation in Tuxedni Group. Bedding } 337^{\circ} / 31^{\circ} \mathrm{W} \text {; pelecypods abundant in some layers; mainly sand- } \\
\text { stone, some siltstone. General description of Tuxedni Group view across valley to northeast: } \\
\text { 1. Upper one-third: Tan to tan-orange siltstones and sandstone. } \\
\text { 2. Middle one-third: Variegated gray, white, dark gray, and orange-tan siltstone, shales, and sandstones. Top to bottom: } \\
\text { gray, white, gray, dark gray, white, gray to brown-gray, tan-orange, dark gray, white, gray, dark gray, tan, white, gray to } \\
\text { bottom one-third of unit. } \\
\text { 3. Lower one-third: Tan-orange siltstone and sandstone with a dark gray unit running through the middle. } \\
\text { Elevation, top of hill: } 4,500 \text { feet. Bedding (halfway between B- } 23 \text { and hilltop) } 327^{\circ} / 39^{\circ} \mathrm{N} \text {; nautiloids fairly common. }\end{array}$ \\
\hline B-24 & & $\begin{array}{l}1351^{\circ}{ }^{\circ} \text { bearing } \\
\text { to camp }\end{array}$ & 6 & 439729 & 6865187 & $\begin{array}{l}\text { Elevation: } 3,650 \text { feet } \\
\text { Diabase dike, trend } \sim 299^{\circ} \text {. Strike and dip } 111^{\circ} / 77^{\circ} \mathrm{S} \text { (straight average of three readings). True thickness } \sim 40 \text { feet; goes up to } \\
\text { elevation } 4,150 \text { feet; hooks slightly north. }\end{array}$ \\
\hline B-25 & & & 6 & 439511 & 6865273 & $\begin{array}{l}\text { Elevation: } 4,180 \text { feet } \\
\text { Bearing } 75^{\circ} \text { to top of dike; fault } 060^{\circ} \text {; dips slightly east; shear fractures } 282^{\circ} / 72^{\circ} \mathrm{N} ; 105^{\circ} / 75^{\circ} \mathrm{N} ; 359^{\circ} / 57^{\circ} \mathrm{E}\end{array}$ \\
\hline
\end{tabular}


CASTLE MOUNTAIN - CARIBOU FAULT SYSTEM FIELD NOTES (referenced to station localities - UTM NAD27 CONUS)

by William A. Fuchs

\begin{tabular}{|c|c|c|c|c|c|c|}
\hline NOTE & SAMPLE & LOCATION & ZN & EASTING & NORTHING & DESCRIPTION \\
\hline B-26 & & $\begin{array}{l}\text { Bearing from } \\
\text { camp } 308^{\circ}\end{array}$ & 6 & 439337 & 6865424 & $\begin{array}{l}\text { Elevation: 4,630 feet } \\
\text { Altered diabase dike; strike and dip } 242^{\circ} / 56^{\circ} \mathrm{N} \text { - goes all across cirque; } 20 \text { feet thick; may be a sill; no bedding apparent from } \\
\text { B-25 to here; rocks (Matanuska argillite, mainly) consistently more beat up and fractured than at elevation of B- } 24 \text {. Thrust } \\
\text { faulting?? }\end{array}$ \\
\hline B-27 & & & 6 & 439216 & 6865405 & $\begin{array}{l}\text { Elevation: 4,780 feet } \\
\text { Matanuska Formation near Caribou fault; bedding destroyed }\end{array}$ \\
\hline B-28 & & & 6 & 439131 & 6865292 & $\begin{array}{l}\text { Elevation: } 5,030 \text { feet } \\
\text { Bedding } 060^{\circ} / 51^{\circ} \mathrm{W} \text {; Matanuska Formation }\end{array}$ \\
\hline B-29 & & $\begin{array}{l}\text { Accurate on } \\
\text { map }\end{array}$ & 6 & 438838 & 6865361 & $\begin{array}{l}\text { Intensely sheared Matanuska argillite with tight, very small (wavelength } 2 \text { inches) folds with vertical axes, indicating strike- } \\
\text { slip motion (sense not certain); trend same as ridge trend here. }\end{array}$ \\
\hline B-30 & & $\begin{array}{l}\text { As located on } \\
\text { map }\end{array}$ & 6 & 438658 & 6865248 & Bedding $161^{\circ} / 22^{\circ} \mathrm{N}$ in Matanuska Formation \\
\hline B-31 & & & 6 & 440107 & 6865361 & $\begin{array}{l}\text { Elevation: } 3,260 \text { feet } \\
\text { Moderate-large fault running along gabbro dike, trend } 298^{\circ} \text {. Bedding ( } 25 \text { feet lower) within middle fault block; } 011^{\circ} / 15^{\circ} \mathrm{W} \text { in } \\
\text { Matanuska Formation; coarse biotitic sandstone; } 285^{\circ} / 80^{\circ} \mathrm{S}-\text { calcite-filled shear; probably best estimate of true strike and } \\
\text { dip of fault; prominent fracturing } 045^{\circ} / 69^{\circ} \mathrm{E} \text {; pelecypods; second fault } \sim 100 \text { feet to south, also follows dike, } 279^{\circ} / 83^{\circ} \mathrm{S}, \text { trend } \\
290^{\circ} \text {; south block dropped down; actually the fault is a zone about } 35 \text { feet wide; on south side attitude of } 296^{\circ} / 63^{\circ} \mathrm{S} \text { is clear } \\
\text { on fault plane but curves in dip somewhat; small bed dragged up in fault zone clearly indicates south block down, } 50 \text { foot } \\
\text { displacement is indicated by elevation reading; bedding south of fault is } 047^{\circ} / 8^{\circ} \mathrm{W} \text {. }\end{array}$ \\
\hline B-32 & & & 6 & 437697 & 6861723 & $\begin{array}{l}\text { Elevation: } 3,030 \text { feet } \\
\text { Bedding } 067^{\circ} / 11^{\circ} \mathrm{N} \text {; Talkeetna Formation; submarine volcanic sandstone, medium grained, small scale cross-bedding; very- } \\
\text { well-bedded sequence of volcanic sandstones and tuffs here and above; } 100 \text { feet downstream there is beautiful agglomerate; } \\
\text { fault near here has } 100 \text { foot displacement. }\end{array}$ \\
\hline B-33 & $\begin{array}{l}\begin{array}{l}\text { Nine } \\
\text { samples } \\
\text { total }\end{array} \\
\text { SITE I } \\
\text { PAL-1-I-1 } \\
\text { PAL-1-I-2 } \\
\text { PAL-1-I-3 } \\
\text { SITE II } \\
\text { PAL-1-II-1 } \\
\text { PAL-1-II-2 } \\
\text { PAL-1-II-3 } \\
\text { SITE III } \\
\text { PAL-1-III-1 } \\
\text { PAL-1-III-2 } \\
\text { PAL-1-III-3 }\end{array}$ & $\begin{array}{l}\text { SITE II is } 47 \text { feet } \\
\text { upstream from } \\
\text { Site I } \\
\text { SITE III is } 50 \text { feet } \\
\text { upslope from } \\
\text { Site II }\end{array}$ & 6 & 437605 & 6861914 & $\begin{array}{l}\text { Elevation: } 3,230 \text { feet (mapping done } 7 / 3 / 78 \text { ) } \\
\text { Contact with intrusive stock; felsite (probable rhyolite), aphanitic with very few quartz and chlorite (after mica) phenocrysts; } \\
\text { satiny white, but elsewhere pink (white only near contact?); fantastic cooling joints characterize the whole intrusive; very } \\
\text { minor pyrite; highly baked contact } 6-12 \text { inches thick; Talkeetna Formation; volcanic flow below contact. (Paleomagnetic } \\
\text { sampling of felsic intrusive done on } 7 / 17 / 78 \text {.) Oriented samples. } \\
\text { SITE I: All samples taken within } 7 \text { feet of one another (chill zone) } \\
\text { PAL-1-I-1: } 292^{\circ} / 55 \mathrm{~N}-7 \text { inches above contact } \\
\text { PAL-1-I-2: } 291^{\circ} / 67^{\circ} \mathrm{N}-12 \text { inches above contact (on tape line is } 292^{\circ} \text { ) } \\
\text { PAL-1-I-3: } 294^{\circ} / 88^{\circ} \mathrm{N}-13 \text { inches above contact } \\
\text { SITE II: All samples taken within a } 6 \text { foot area } ~ 8 \text { feet above contact } \\
\text { PAL-1-II-1: Line on top (not strike line) is } 261^{\circ} \\
\text { PAL-1-II-2: Line on top is } 270^{\circ} \\
\text { PAL-1-II-3: } 325^{\circ} / 75^{\circ} \mathrm{N} \\
\text { SITE III: All samples taken within } 6 \text { foot area } \\
\text { PAL-1-III-1: } 0^{\circ} / 77^{\circ} \mathrm{E} \text { PAL-1-III- } \\
\text { 2: } 358^{\circ} / 85^{\circ} \mathrm{E} \text { PAL-1-III-3: } \\
357^{\circ} / 84^{\circ} \mathrm{E}\end{array}$ \\
\hline
\end{tabular}


CASTLE MOUNTAIN - CARIBOU FAULT SYSTEM FIELD NOTES (referenced to station localities - UTM NAD27 CONUS)

by William $A$. Fuchs

\begin{tabular}{|c|c|c|c|c|c|c|}
\hline NOTE & SAMPLE & LOCATION & ZN & EASTING & NORTHING & DESCRIPTION \\
\hline B-34 & $\begin{array}{l}\text { SITE I } \\
\text { PAL-2-I-1 } \\
\text { PAL-2-I-2 } \\
\text { PAL-2-I-3 } \\
\text { SITE II } \\
\text { PAL-2-II-1 } \\
\text { PAL-2-II-2 } \\
\text { PAL-2-II-3 } \\
\text { SITE III } \\
\text { PAL-2-III-1 } \\
\text { PAL-2-III-2 } \\
\text { PAL-2-III-3 }\end{array}$ & $\begin{array}{l}\text { Samples taken } \\
50 \text { feet upslope } \\
\text { from stream } \\
\text { SITE II is } 50 \text { feet } \\
\text { upstream from } \\
\text { site I } \\
\text { SITE III is } 30 \text { feet } \\
\text { upstream from } \\
\text { site II }\end{array}$ & 6 & 437518 & 6862003 & 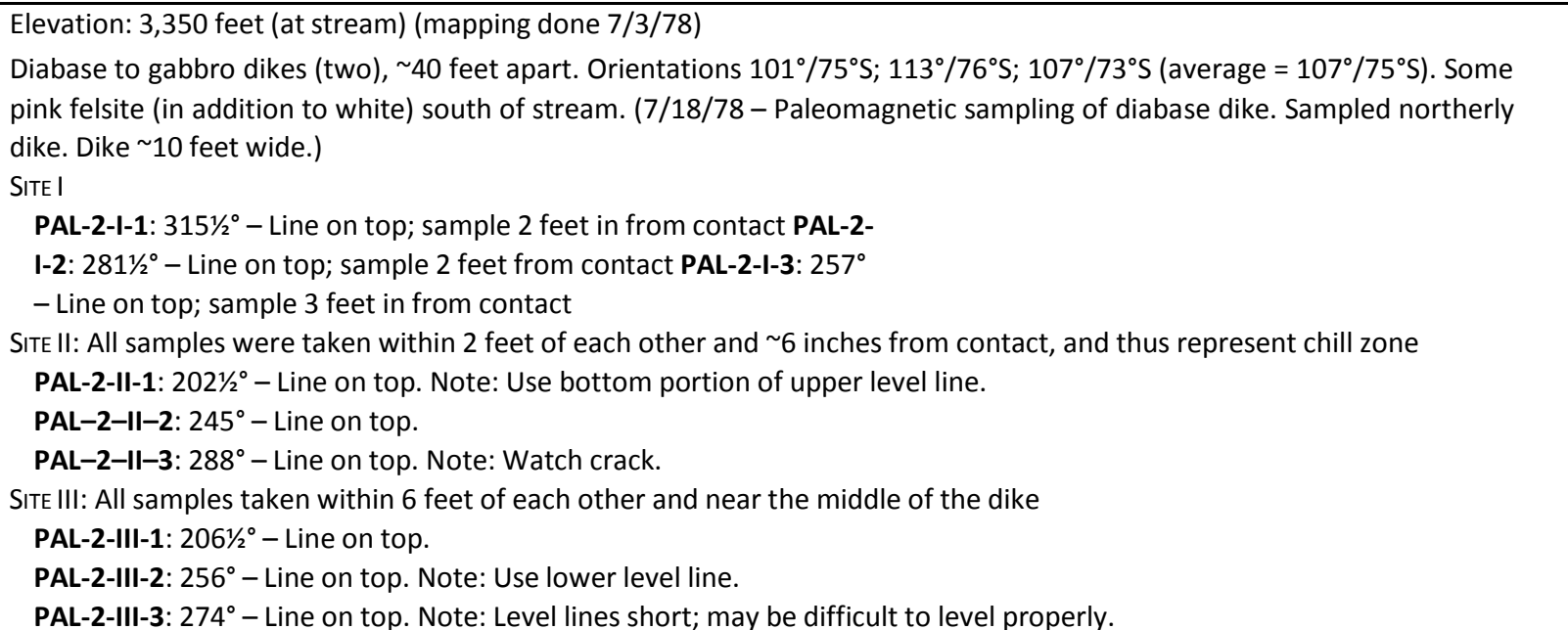 \\
\hline B-35 & $\begin{array}{l}\text { B-2 and } \\
\text { B-2-Am }\end{array}$ & $\begin{array}{l}\text { 150 feet up- } \\
\text { stream from } \\
\text { B-34; north side } \\
\text { of stream }\end{array}$ & 6 & 437463 & 6862035 & $\begin{array}{l}\text { Elevation: } 3,400 \text { feet } \\
\text { Somewhat coarser variety of rhyolite; white with uniform distribution of green poikiloblastic hornblende?; chlorite, very minor } \\
\text { pyrite? Samples B-2 and B-2-Am for age dating. } \\
\text { [Determined to be rhyolite by geochemical whole rock analysis done after dissertation completed. See analyses.] }\end{array}$ \\
\hline B-36 & & & 6 & 440321 & 6865815 & $\begin{array}{l}\text { Elevation: } 3,260 \text { feet } \\
\text { Clam fossils in sandstone; also limestone nearby; Matanuska Formation. }\end{array}$ \\
\hline B-37 & & & 6 & 439934 & 6866133 & $\begin{array}{l}\text { Elevation: } 3,230 \text { feet } \\
\text { Eastern limit of exposed Chickaloon?; pebble conglomerate; actually this is located float. }\end{array}$ \\
\hline B-38 & & & 6 & 439780 & 6866111 & $\begin{array}{l}\text { Elevation: } 3,420 \text { feet } \\
\text { In float - fault gouge characteristic of that found in the Caribou fault; Caribou fault probably very close to this spot. }\end{array}$ \\
\hline B-39 & & & 6 & 439641 & 6866158 & $\begin{array}{l}\text { Elevation: } 3,390 \text { feet } \\
\text { Bedding } 112^{\circ} / 24^{\circ} \mathrm{N} \text {; in Tuxedni Group? Thick sequence of sandstones with considerable volcanic material. }\end{array}$ \\
\hline B-40 & $\begin{array}{l}\text { B-3 } \\
\text { B-3-Am } \\
\text { B-3-2 }\end{array}$ & & 6 & 439520 & 6866062 & $\begin{array}{l}\text { Elevation: } 3,650 \text { feet } \\
\text { Caribou fault zone, } \sim 50-100 \text { feet wide, Chickaloon pebble conglomerate to south; Tuxedni Group sandstone to north; } \\
\text { beautiful fault gouge (samples). fault trend }=085^{\circ} \text {. Samples B-3, B-3-Am, and B-3-2 for possible future age dating. }\end{array}$ \\
\hline B-41 & & & 6 & 439218 & 6865929 & $\begin{array}{l}\text { Elevation: } 4,040 \text { feet } \\
\text { Caribou fault zone; well exposed on ridge; fault trend }=262^{\circ} .\end{array}$ \\
\hline B-42 & & & 6 & 439104 & 6865941 & $\begin{array}{l}\text { Elevation: } 4,010 \text { feet } \\
\text { Caribou fault? Zone } 082^{\circ} / 25^{\circ} \mathrm{S} \text { is attitude on shear zone; Tuxedni Group north of fault; Talkeetna Formation south of fault; } \\
\text { attitude of fault roughly parallels bedding in Tuxedni; rotated fault zone?? }\end{array}$ \\
\hline B-43 & & & 6 & 439183 & 6865817 & $\begin{array}{l}\text { Elevation: } 4,220 \text { feet } \\
\text { Fault; Chickaloon Formation to south and Tuxedni Group to north, fault zone } 50 \text { feet wide; diabase dike occupies zone and } \\
\text { its trend appears to be trend of fault } 230^{\circ} \text {. }\end{array}$ \\
\hline
\end{tabular}


CASTLE MOUNTAIN - CARIBOU FAULT SYSTEM FIELD NOTES (referenced to station localities - UTM NAD27 CONUS)

by William A. Fuchs

\begin{tabular}{|c|c|c|c|c|c|c|}
\hline NOTE & SAMPLE & LOCATION & ZN & EASTING & NORTHING & DESCRIPTION \\
\hline B-44 & $\begin{array}{l}\text { B-5 } \\
\text { B-5-Am }\end{array}$ & & 6 & 439126 & 6865657 & $\begin{array}{l}\text { Elevation: } 4,310 \text { feet } \\
\text { Bedding } 061^{\circ} / 48^{\circ} \mathrm{N} \text {; Chickaloon Formation, pebble conglomerate. }\end{array}$ \\
\hline B-45 & & & 6 & 439116 & 6865609 & $\begin{array}{l}\text { Elevation: } 4,345 \text { feet } \\
\text { Bedding } 319^{\circ} / 45^{\circ} \mathrm{N} \text {; Chickaloon Formation; fault between here and B-44. }\end{array}$ \\
\hline B-46 & $\begin{array}{l}\text { B-4 } \\
\text { B-4-Am }\end{array}$ & & 6 & 439102 & 6865433 & $\begin{array}{l}\text { Elevation: } 4,620 \text { feet } \\
\text { Contact of Chickaloon Formation (pebble-cobble conglomerate, } ~ 50 \text { feet thick) with Matanuska Formation argillite. Unfor- } \\
\text { tunately, only Chickaloon Formation is exposed in outcrop, but this does not appear to be a fault contact. Looking westward } \\
\text { along contact toward knob } 2,000 \text { feet westward, a consistent strike and dip in the Matanuska Formation can be seen, meas- } \\
\text { ured as } 242^{\circ} / 65^{\circ} \mathrm{N} \text { on a resistant unit. This is the Matanuska-Chickaloon sequence Detterman and others (1976) match up } \\
\text { with a similar sequence on the north side of the fault to obtain } 16 \text { ? kilometer right lateral offset. Samples B-4 and B-4Am of } \\
\text { Chickaloon Formation; pebble conglomerate plus a curious calcareous concretionary? rock, which weathers orange, found } \\
\text { sporadically in the same unit. Walking eastward downstream it can be seen that Matanuska Formation at the contact is a fairly } \\
\text { thick, black argillite sequence with numerous limestone concretion layers (which fizz in HCl). Here, the Chickaloon Formation is } \\
\text { not pebble-cobble conglomerate as in Sample B-4, but rather a pebble conglomerate as in Samples B-5 and B- 5-Am at site B- } \\
\text { 44. Samples B-4 and B-4-Am for comparison purposes. }\end{array}$ \\
\hline B-46-X & & & 6 & 439419 & 6866824 & $\begin{array}{l}\text { Elevation: 3,070 feet } \\
\text { Cataclasite, Horn Mountain Tuff member of the Talkeetna Formation-large fault zone, exposed for a distance of } 300 \text { feet } \\
\text { upstream; slickensides indicate more strike-slip than dip-slip motion; fault has a very rough trend of } 320^{\circ} \text { at this point (if the } \\
\text { slickenside surfaces are a true indication of the fault trend) }\end{array}$ \\
\hline B-47 & & & 6 & 439007 & 6866223 & $\begin{array}{l}\text { Elevation: } 3,490 \text { feet } \\
\text { Tuxedni Group, sandstone. Bedding } 127^{\circ} / 23^{\circ} \mathrm{N} \text {; small? fault } 20 \text { feet to the south, attitude } 085^{\circ} / 57^{\circ} \mathrm{N} \text {; prominent fractures } \\
085^{\circ} / 53^{\circ} \mathrm{N}-\text { parallels fault, } 189^{\circ} \text { nearly vertical. Fault farther upstream, elevation } 3,570 \text { feet; strike changes from } 050^{\circ} \text { east } \\
\text { of stream to } 064^{\circ} \text { west of stream; dip } 72^{\circ} \mathrm{S} \text {. Farther upstream, elevation } 3,590 \text { feet; small intrusive, } 30 \text { foot diameter, diorite, } \\
\text { speckled gray, equigranular, } 60 \% \text { subhedral, milky plagioclase, } 30 \% \text { anhedral green hornblende, }>10 \% \text { (close to } 10 \% \text { ) } \\
\text { subhedral quartz; medium-grained, small calcite veinlets, somewhat pyritic in places, may correlate with diorite pluton up } \\
\text { Kings River. }\end{array}$ \\
\hline B-48 & & & 6 & 438918 & 6866027 & $\begin{array}{l}\text { Elevation: } 3,730 \text { feet } \\
\text { Fault gouge, fault may roughly parallel stream here }\end{array}$ \\
\hline B-49 & & & 6 & 438896 & 6865662 & $\begin{array}{l}\text { Elevation: } 4,150 \text { feet } \\
\text { Fault breccia, } 4 \text { feet wide, quartz cementing Tuxedni fragments, trend } 355^{\circ} \\
\text { Note from } 7 / 7 / 79 \text { : South of B- } 49 \text { in north-trending fault zone in Talkeetna Formation. Slick surface }=15^{\circ} / 58^{\circ} \mathrm{W} \text {; slickensides } \\
=16^{\circ} \text { plunge, } 218^{\circ} \text { bearing; east of zone } 247^{\circ} / 66^{\circ} \mathrm{N} \text { surface, horizontal slickenside. }\end{array}$ \\
\hline B-50 & & & 6 & 438833 & 6865648 & $\begin{array}{l}\text { Elevation: 4,190 feet } \\
\text { Tuxedni Group sandstone, highly fractured. Elevation: 4,270 feet (above lower outcrop). Mixed Tuxedni Group and diabase } \\
\text { dike rock (scrambled diabase dike occurs throughout the fault zones in this area) }\end{array}$ \\
\hline B-51 & & & 6 & 438689 & 6865401 & $\begin{array}{l}\text { Elevation: } 4,590 \text { feet } \\
\text { Lower contact Chickaloon Formation, fault contact with Talkeetna Formation to the south. }\end{array}$ \\
\hline B-52 & & & 6 & 438544 & 6865604 & $\begin{array}{l}\text { Elevation: } 4,240 \text { feet } \\
\text { Chickaloon Formation, next to fault, Talkeetna Formation east of fault }\end{array}$ \\
\hline B-53 & & & 6 & 438667 & 6866067 & $\begin{array}{l}\text { Elevation: } 3,780 \text { feet } \\
\text { Fault zone, Talkeetna Formation on both sides, trend on fault from shear zone in stream }=230^{\circ} \text {; going northward found hor- } \\
\text { rendous fault breccia with calcite filling in vugs - possibly a very large fault }\end{array}$ \\
\hline
\end{tabular}


CASTLE MOUNTAIN - CARIBOU FAULT SYSTEM FIELD NOTES (referenced to station localities - UTM NAD27 CONUS)

by William A. Fuchs

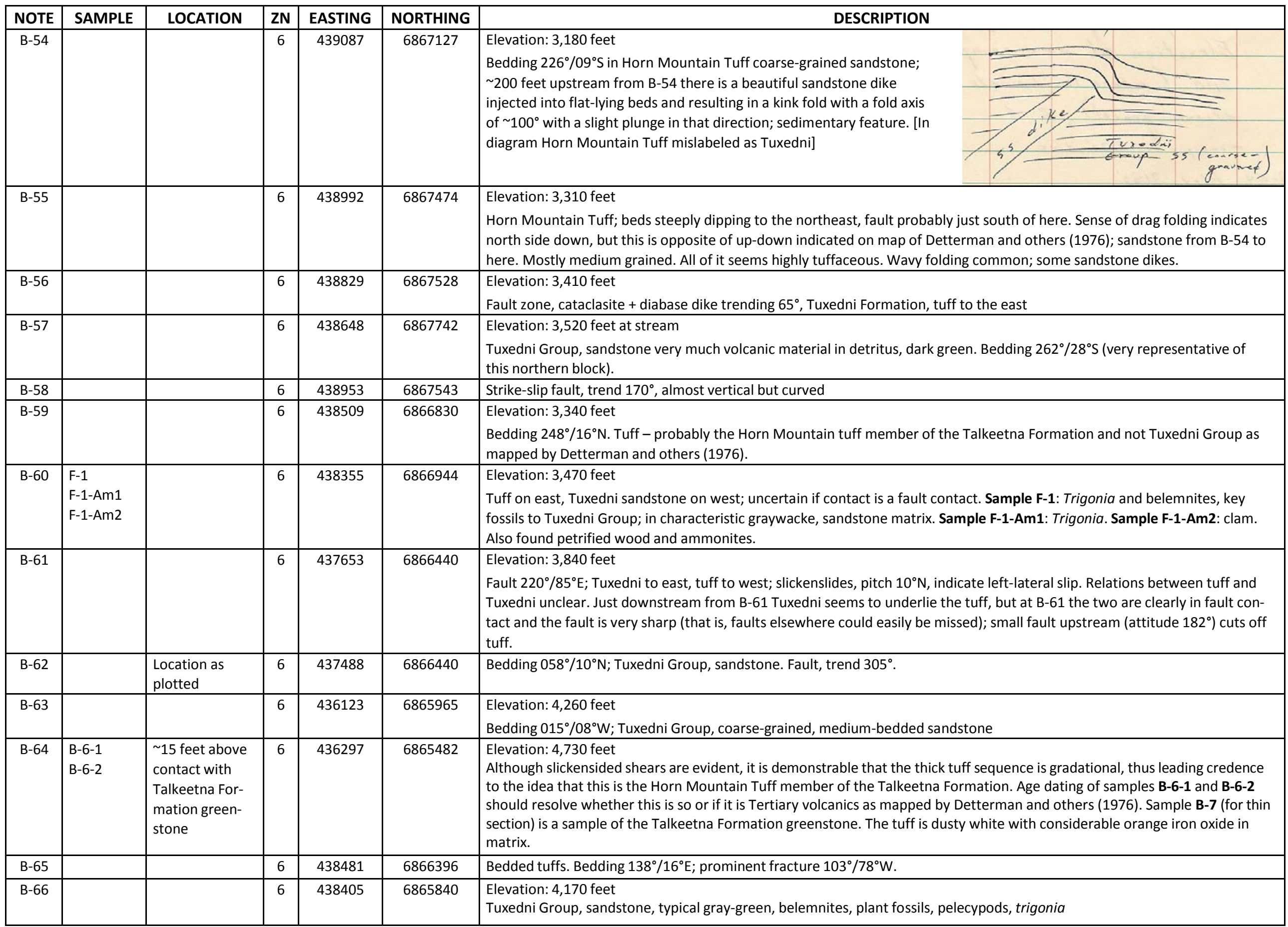


CASTLE MOUNTAIN - CARIBOU FAULT SYSTEM FIELD NOTES (referenced to station localities - UTM NAD27 CONUS)

by William A. Fuchs

\begin{tabular}{|c|c|c|c|c|c|c|}
\hline NOTE & SAMPLE & LOCATION & ZN & EASTING & NORTHING & DESCRIPTION \\
\hline B-67 & & & 6 & 438142 & 6865405 & $\begin{array}{l}\text { Elevation: } 4,810 \text { feet } \\
\text { Talkeetna Formation greenstones to south and Talkeetna Formation (I think it is) pink, porphyritic, less altered volcanic flows } \\
\text { to the north. }\end{array}$ \\
\hline B-68 & & & 6 & 437842 & 6865120 & $\begin{array}{l}\text { Elevation: } 4,440 \text { feet } \\
\text { Bedding } 145^{\circ} / 10^{\circ} \mathrm{W} \text {; Chickaloon Formation. Important location because Chickaloon- } \\
\text { Matanuska contact is seen. Looking northeast from here Chickaloon is a } 350 \text { foot } \\
\text { sequence of almost entirely pebble conglomerate, with pebbles of black chert, white } \\
\text { quartz, and Talkeetna? Formation, (plus some pebble-cobble conglomerate). It is } \\
\text { steeply dipping southward along fault. Actual contact with Matanuska is occupied by } \\
\text { dike-sills of diabase. Contact was probably originally erosional with Chickaloon, forming } \\
\text { stream gravels. The upper Matanuska is a dark argillite with some sandstone beds. No fossils seen. }\end{array}$ \\
\hline B-69 & & & 6 & 437328 & 6865010 & $\begin{array}{l}\text { Elevation: } 4,850 \text { feet } \\
\text { Caribou fault zone starts here and continues upstream. Very well exposed and sharp. Attitude on fault plane } 240^{\circ} / 15^{\circ} \mathrm{N}- \\
\text { actually a thrust here, but this is probably not true for whole zone. }\end{array}$ \\
\hline B-70 & $\begin{array}{l}B-8-1 \\
B-8-2\end{array}$ & & 6 & 437077 & 6864735 & $\begin{array}{l}\text { Elevation: } 5,480 \text { feet } \\
\text { In fault zone: diabase-gabbro dike, attitude } 115^{\circ} / 70^{\circ} \text { S; representative of many dikes. Samples B-8-1 and B-8-2 for age dating. }\end{array}$ \\
\hline B-71 & & & 6 & 437192 & 6864662 & $\begin{array}{l}\text { Elevation: } 5,650 \text { feet, ridge top } \\
\text { Strongly cross-bedded; } 63^{\circ} / 63^{\circ} \mathrm{N} \text {; Chickaloon Formation }\end{array}$ \\
\hline B-72 & & & 6 & 437074 & 6864429 & Chickaloon Formation. Bedding $074^{\circ} / 40^{\circ} \mathrm{N}$; nearby dike oriented $267^{\circ} / 54^{\circ} \mathrm{S}$. \\
\hline B-73 & & & 6 & 441855 & 6866887 & $\begin{array}{l}\text { Elevation: } 3,290 \text { feet } \\
\text { Bedding } 164^{\circ} / 25^{\circ} \mathrm{E} \text {; Chinitna Formation, siltstone, indurated, fractured, indistinguishable from Tuxedni Group. }\end{array}$ \\
\hline B-74 & & & 6 & 441866 & 6867027 & $\begin{array}{l}\text { Elevation: } 3,670 \text { feet } \\
\text { Bedding } 254^{\circ} / 19^{\circ} \mathrm{S} \text {; Chinitna Formation; from B-73 to here has been a continuous section of greenish gray to brown-gray } \\
\text { siltstone (with some limestone concretionary layers) with very occasional thin sandstone beds. No fossils seen. }\end{array}$ \\
\hline B-75 & & & 6 & 441482 & 6867166 & $\begin{array}{l}\text { Elevation: } 3,050 \text { feet } \\
\text { Bedding } 205^{\circ} / 22^{\circ} \mathrm{E} \text {; Chinitna Formation, siltstone, brownish gray with concretions, thin, medium-grained sandstone, orange }\end{array}$ \\
\hline B-76 & & & 6 & 441175 & 6868000 & $\begin{array}{l}\text { Elevation: } 3,260 \text { feet } \\
\text { Fault zone, attitude } 168^{\circ} / 75^{\circ} \mathrm{W} \text {; normal fault; abruptly cuts off Tuxedni Group sandstone; gray with minor conglomerate and } \\
\text { belemnites; in places it is adjacent to orange Tuxedni siltstone. }\end{array}$ \\
\hline B-77 & & & 6 & 441560 & 6868526 & Fault seen from opposite ridge, displacement roughly 400 feet Note: from ridge to peak $=236^{\circ}$ at elevation 3,970 feet \\
\hline
\end{tabular}


CASTLE MOUNTAIN - CARIBOU FAULT SYSTEM FIELD NOTES (referenced to station localities - UTM NAD27 CONUS)

by William A. Fuchs

\begin{tabular}{|c|c|c|c|c|c|c|}
\hline NOTE & SAMPLE & LOCATION & ZN & EASTING & NORTHING & DESCRIPTION \\
\hline B-78 & & & 6 & 441661 & 6868457 & $\begin{array}{l}\text { Elevation: } 4,130 \text { feet } \\
\text { Bedding } 181^{\circ} / 24^{\circ} \mathrm{E} \text {; Chinitna Formation, dark sandstone, concretions, some pyritic areas, lower boundary of formation is at } \\
\sim 4,070 \text { feet. Tuxedni below is brown (weathered) siltstone hundreds of feet thick with concretionary layers, very uniform. } \\
\text { Story of the Tuxedni-Chinitna contact: As mapped by Detterman and others (1976) at this location, the uppermost Tuxedni } \\
\text { Group consists of hundreds of feet ( }>500 \text { feet) of non-resistant, orange-brown weathering siltstone with concretionary } \\
\text { layers, all very uniform in appearance. The Chinitna Formation is concordant and begins with a dark brown, medium-grained } \\
\text { sandstone, perhaps } 50-100 \text { feet thick, then a brown siltstone, non-resistant, less orange (weathered) than the uppermost } \\
\text { Tuxedni, perhaps } 100-200 \text { feet thick. Then a very thick sequence ( }>1,000 \text { feet) of resistant, cliff-forming black and white } \\
\text { monotonous, alternating rocks, siltstone with thin sandstone interbeds. The uppermost rocks are predominantly gray silt- } \\
\text { stone with one distinctive orange sandstone bed. Belemnites are moderately common in the Chinitna and less common are } \\
\text { beds with pelecypods. Occasional ammonites are also found. The Chinitna may be very difficult to distinguish from Tuxedni } \\
\text { in isolated outcrops. (Note added } 9 / 9 / 78 \text { ) Except for the lowermost beds, the Chinitna is noticeably less fossiliferous than } \\
\text { the Tuxedni Group. }\end{array}$ \\
\hline B-79 & & & 6 & 442496 & 6867167 & $\begin{array}{l}\text { Elevation: } 3,250 \text { feet } \\
\text { Bedding } 169^{\circ} / 16^{\circ} \mathrm{E} \text {; Tuxedni Group; no fossils seen. Greenish gray sandstone like Tuxedni Group. }\end{array}$ \\
\hline B-80 & & & 6 & 442629 & 6867581 & $\begin{array}{l}\text { Elevation: } 3,470 \text { feet } \\
\text { Bedding } 027^{\circ} / 13^{\circ} \mathrm{E} \text {; Tuxedni Group }\end{array}$ \\
\hline B-81 & & & 6 & 442074 & 6867847 & Pronounced unconformity between Chickaloon and Chinitna \\
\hline B-82 & SK-1 & $\begin{array}{l}\text { West side of } \\
\text { King River }\end{array}$ & 6 & 413952 & 6856740 & $\begin{array}{l}\text { Elevation: } 3,410 \pm 30 \text { feet } \\
\text { Sample SK-1, two bags for age dating; sheared diorite. Shear fractures } 323^{\circ} / 88^{\circ} \mathrm{E}-\text { sampled; shear fractures } 336^{\circ} / 88^{\circ} \mathrm{E} \text {; } \\
332^{\circ} / 80^{\circ} \mathrm{E} ; 337^{\circ} / 45^{\circ} \mathrm{W} ; 295^{\circ} / 85^{\circ} \mathrm{N} \text {; taken } \sim 300 \text { feet from main Castle Mountain fault zone; many shears slickensided. }\end{array}$ \\
\hline B-83 & SK-2 & & 6 & 413988 & 6856764 & $\begin{array}{l}\text { Elevation: } 3,470 \pm 30 \text { feet } \\
\text { Sample SK-2, two bags for age dating. Shear fractures } 330^{\circ} / 75^{\circ} \mathrm{E} \text { (sampled); } 328^{\circ} / 85^{\circ} \mathrm{W} ; 080^{\circ} / 10^{\circ} \mathrm{N} \text { - many approximately } \\
\text { parallel to this. }\end{array}$ \\
\hline B-84 & SK-3 & $\begin{array}{l}\text { Bearing } 252^{\circ} \text { to } \\
\text { peak across } \\
\text { river }\end{array}$ & 6 & 413563 & 6857358 & $\begin{array}{l}\text { Elevation: } 3,730 \text { feet } \\
\text { Sample SK-3, for age dating. Fresh diorite, medium-grained, some calcite veinlets. }\end{array}$ \\
\hline B-85 & SK-4 & $\begin{array}{l}\text { Bearing } 246^{\circ} \text { to } \\
\text { peak across } \\
\text { river }\end{array}$ & 6 & 413314 & 6857531 & $\begin{array}{l}\text { Elevation: } 3,700 \pm 30 \text { feet } \\
\text { Sample SK-4, for age dating, fresh diorite, more mafics and pinker than SK-3, some slickenside fractures nearby, but these } \\
\text { caused little or no alteration in the samples taken. } \\
\text { NOTE: The purpose of age dating samples SK- } 1 \text { through SK- } 4 \text { is to compare the fission track age dates of apatite in the sheared } \\
\text { diorite with that in fresh diorite. Apatite fission tracks anneal at } 150^{\circ} \mathrm{C} \text { and thus it is possible that a significant faulting event } \\
\text { accompanied by frictional heating may be dated. }\end{array}$ \\
\hline B-86 & & & 6 & 414050 & 6856443 & Matanuska Formation, siltstone, dark gray, highly fractured, no fossils seen. \\
\hline B-87 & & & 6 & 440738 & 6864305 & $\begin{array}{l}\text { Elevation: } 3,210 \text { feet } \\
\text { Lowest point on spectacular diabase dike on east side of Boulder Creek across from camp; orientation of cooling joints } 136^{\circ} / \\
\text { vertical. Rough trend on dike } 127^{\circ} \text {. }\end{array}$ \\
\hline B-88 & & & 6 & 440863 & 6864365 & $\begin{array}{l}\text { Elevation: } 3,350 \text { feet } \\
\text { Bedding or foliation (cleavage?) } 013^{\circ} / 51^{\circ} \mathrm{W} \text {; probably an altered (it has pyrite and some sericitic sheen) welded tuff next to } \\
\text { diabase dike. Just upslope from here at an elevation of } 3,420 \text { feet is a dike swarm } 100 \text { feet wide with an orientation of } \\
120^{\circ} / 77^{\circ} \mathrm{S} \text {; fairly certain dike occupies a fault as evidenced by sheared material almost gouge-like. }\end{array}$ \\
\hline
\end{tabular}


CASTLE MOUNTAIN - CARIBOU FAULT SYSTEM FIELD NOTES (referenced to station localities - UTM NAD27 CONUS)

by William A. Fuchs

\begin{tabular}{|c|c|c|c|c|c|c|}
\hline NOTE & SAMPLE & LOCATION & ZN & EASTING & NORTHING & DESCRIPTION \\
\hline B-89 & & & 6 & 441040 & 6864419 & $\begin{array}{l}\text { Elevation: } 3,550 \text { feet } \\
\text { Diabase dike - attitude } 103 \% \text { approximately vertical; } ~ 50 \text { feet wide }\end{array}$ \\
\hline B-90 & & & 6 & 441472 & 6864367 & $\begin{array}{l}\text { Elevation: } 4,410 \text { feet } \\
\text { Fault, right-lateral strike-slip offsets distinctive dike swarm } 500 \text { feet; can be seen to extend southward across valley; trend } \\
010^{\circ} \text {; must have some normal vertical component with east-side-up (as indicated by } 500 \text { foot gap in the dike swarm); dike } \\
\text { occupies fault at this location. }\end{array}$ \\
\hline B-91 & $\begin{array}{l}\text { B-9-Am-I } \\
\text { B-9-Am-II } \\
\text { fossils }\end{array}$ & & 6 & 441770 & 6864341 & Talkeetna Formation - high-rank graywacke, highly fossiliferous, mostly mollusks, some ammonite fragments. \\
\hline B-92 & & & 6 & 441129 & 6865404 & $\begin{array}{l}\text { Elevation: } 3,080 \text { feet } \\
\text { Bedding } 061^{\circ} / 40^{\circ} \text {. Matanuska Formation, mostly well-indurated siltstone, bedding reading taken on small sandstone bed. No } \\
\text { fossils seen to this point. }\end{array}$ \\
\hline B-93 & & & 6 & 441266 & 6865393 & $\begin{array}{l}\text { Elevation: } 3,240 \text { feet } \\
\text { Diabase dike, trend } 123^{\circ} \text {, nearly vertical. }\end{array}$ \\
\hline B-94 & & & 6 & 441467 & 6865452 & $\begin{array}{l}\text { Elevation: } 3,560 \text { feet } \\
\text { Diabase dike, } 3 \text { feet wide, trend } 126^{\circ} \text {, nearly vertical. }\end{array}$ \\
\hline B-95 & & & 6 & 441497 & 6865410 & $\begin{array}{l}\text { Elevation: } 3,660 \text { feet } \\
\text { Diabase dike, trend } 105^{\circ} \text {, nearly vertical. Fault } 75 \text { feet southeast along ridge from here separates Matanuska Formation on } \\
\text { north from Talkeetna Formation, silicified tuff on south; fault trend } 055^{\circ} \text {. }\end{array}$ \\
\hline B-96 & & & 6 & 441616 & 6865153 & $\begin{array}{l}\text { Elevation: } 4,065 \text { feet } \\
\text { Cataclastic fault zone, trends } 090^{\circ} \text { to } \mathrm{W}, 095^{\circ} \text { to E. Prominent faults } 132^{\circ} ; 172^{\circ} \text {. Slickensides dominantly strike-slip; } 310^{\circ} \\
\text { slickensides. }\end{array}$ \\
\hline B-97 & & & 6 & 441616 & 6865118 & $\begin{array}{l}\text { Elevation: } 4,140 \text { feet } \\
\text { Diabase dike, trend } 102^{\circ} \text {, nearly vertical }\end{array}$ \\
\hline B-98 & & & 6 & 440374 & 6863679 & $\begin{array}{l}\text { Elevation: } 3,080 \text { feet } \\
\text { Diabase dike; trend } 100^{\circ}\end{array}$ \\
\hline B-99 & & & 6 & 440593 & 6863514 & $\begin{array}{l}\text { Elevation: } 3,310 \text { feet } \\
\text { Diabase dike swarm } 100 \text { feet wide, rough trend } 150^{\circ} \pm 5^{\circ} \text {. Note topo map is screwed-up here. Stream fork is mislocated by at } \\
\text { least } 200 \text { feet. }\end{array}$ \\
\hline B-100 & & & 6 & 440702 & 6863681 & $\begin{array}{l}\text { Elevation: } 3,480 \text { feet } \\
\text { Bedding } 092^{\circ} / 25^{\circ} \mathrm{N} \text {; Talkeetna Formation, volcanic siltstone; surrounding rocks are flows and agglomerates. Bedding } 100 \\
\text { feet upstream } 087^{\circ} / 14^{\circ} \mathrm{N} \text {. }\end{array}$ \\
\hline B-101 & & & 6 & 438256 & 6863742 & $\begin{array}{l}\text { Elevation: } 4,060 \text { feet } \\
\text { Diabase dike; attitude } 296^{\circ} / 72^{\circ} \mathrm{S}\end{array}$ \\
\hline B-102 & & & 6 & 438232 & 6863801 & $\begin{array}{l}\text { Elevation: } 4,150 \text { feet } \\
\text { Talkeetna Formation-Tuxedni Group contact; fault contact, but fault may be small. Some slickensides. Bedding } 060^{\circ} / 38^{\circ} \mathrm{N} \text { in } \\
\text { Tuxedni Group sandstone; bedding taken } 200 \text { feet west along slope at same elevation as this locality. }\end{array}$ \\
\hline
\end{tabular}


CASTLE MOUNTAIN - CARIBOU FAULT SYSTEM FIELD NOTES (referenced to station localities - UTM NAD27 CONUS)

by William A. Fuchs

\begin{tabular}{|c|c|c|c|c|c|c|}
\hline NOTE & SAMPLE & LOCATION & ZN & EASTING & NORTHING & DESCRIPTION \\
\hline B-103 & $\begin{array}{l}\text { FSB-10 } \\
\text { FSB-11-AM } \\
\text { FSB-11 }\end{array}$ & & 6 & 438117 & 6864092 & $\begin{array}{l}\text { Elevation: } 4,590 \text { feet } \\
\text { Bedding } 036^{\circ} / 37^{\circ} \mathrm{NW} ; 051^{\circ} / 47^{\circ} \mathrm{NW} ; 051^{\circ} / 36^{\circ} \mathrm{NW} \text {. Tuxedni Group. Sample FSB-10 - Very distinctive brachiopod (in field asst. } \\
\text { Steve McMillin's possession). Also good belemnites. Sample FSB-11-AM - mollusks and belemnites. Sample FSB-11 - (in } \\
\text { McMillin's possession) mollusks and belemnites. } \\
\text { 7/11/79: Boulder Creek fault north of B103 has an overall dip of } 50^{\circ} \mathrm{S} \text { (it is actually somewhat undulatory). }\end{array}$ \\
\hline B-104 & & & 6 & 441390 & 6865812 & Bedding $271^{\circ} / 16^{\circ} \mathrm{N}$. Matanuska Formation. \\
\hline B-105 & & & 6 & 442125 & 6865895 & $\begin{array}{l}\text { Elevation: } 3,080 \text { feet } \\
\text { Bedding } 261^{\circ} / 41^{\circ} \mathrm{N} \text {. Matanuska Formation } 150 \text { feet north of major fault separating Matanuska and Talkeetna Formations. }\end{array}$ \\
\hline B-106 & & & 6 & 442421 & 6865614 & $\begin{array}{l}\text { Elevation: } 3,470 \text { feet } \\
\text { Diabase dike - attitude } 098^{\circ} / 77^{\circ} \mathrm{S} \text {; in Talkeetna Formation; part of a small dike swarm; better overall trend is probably } 115^{\circ} \text {. }\end{array}$ \\
\hline B-107 & & & 6 & 442669 & 6865100 & $\begin{array}{l}\text { Elevation: } 3,790 \text { feet } \\
\text { Fault with diabase dike; rough trend } 095^{\circ} \text {; smaller pyritic mineralized zone is associated. }\end{array}$ \\
\hline B-108 & & & 6 & 442844 & 6864761 & $\begin{array}{l}\text { From this point to upstream } 600 \text { feet greenstone exposed similar to that found on Kings River. Section from B-105 to B-108 } \\
\text { represents } \sim 2,000 \text { feet of Talkeetna Formation above greenstone, assuming a northward dip of } 25^{\circ} \text {. }\end{array}$ \\
\hline B-109 & & & 6 & 442892 & 6865454 & Fault seen from a distance; displacement 200 feet, northwest side up. \\
\hline B-110 & & & 6 & 436914 & 6864177 & $\begin{array}{l}\text { Elevation: 5,730 feet } \\
\text { Fault zone, } \sim 150 \text { feet wide, occupied by numerous diabase dikes; trend at this point } 90^{\circ} .\end{array}$ \\
\hline B-111 & SB-12 & & 6 & 436879 & 6864076 & $\begin{array}{l}\text { Elevation: 5,830 feet (topo probably wrong here) } \\
\text { Sample SB-12 - Belemnites in dark gray siltstone (Chinitna? Formation); section composed of predominantly siltstone with } \\
\text { limestone concretionary layers and some thin beds of medium to coarse sandstone with moderate feldspar. }\end{array}$ \\
\hline B-112 & & & 6 & 436762 & 6863940 & $\begin{array}{l}\text { Elevation: } 5,930 \text { feet } \\
\text { Bedding } 078^{\circ} / 35^{\circ} \mathrm{N} \text {; Chinitna? Formation }\end{array}$ \\
\hline B-113 & & & 6 & 436288 & 6864047 & $\begin{array}{l}\text { Elevation: } 5,750 \text { feet } \\
\text { Diabase dike, trend } 125^{\circ}\end{array}$ \\
\hline B-114 & & & 6 & 436315 & 6863981 & $\begin{array}{l}\text { Elevation: 5,800 feet } \\
\text { Bedding } 253^{\circ} / 47 \mathrm{~N} \text {; Chinitna Formation? }\end{array}$ \\
\hline B-115 & & & 6 & 437000 & 6864652 & Small fault (100 foot displacement) in Chickaloon Formation seen across valley from B-114 \\
\hline B-116 & & & 6 & 436599 & 6863621 & $\begin{array}{l}\text { Elevation: } 5,780 \text { feet } \\
\text { Fault with up to } 200 \text { foot displacement }\end{array}$ \\
\hline B-117 & & & 6 & 436815 & 6860597 & $\begin{array}{l}\text { Elevation: } 2,950 \text { feet } \\
\text { Felsite intrusive }\end{array}$ \\
\hline B-118 & & & 6 & 436705 & 6860705 & $\begin{array}{l}\text { Elevation: 3,350 feet } \\
\text { Igneous breccia containing mostly Talkeetna Formation pebbles with small amount of igneous matrix. }\end{array}$ \\
\hline B-119 & & & 6 & 436545 & 6860619 & Felsite intrusive \\
\hline B-120 & & & 6 & 439036 & 6860061 & $\begin{array}{l}\text { Elevation: } 2,990 \text { feet } \\
\text { Talkeetna Formation, maroon agglomerate. }\end{array}$ \\
\hline B-121 & SB-13 & & 6 & 439968 & 6859891 & $\begin{array}{l}\text { Elevation: 3,880 feet } \\
\text { Diorite intrusive, medium-grained; chloritic and cataclastic in places; causes doming-up of overlying Talkeetna Formation. } \\
\text { Sample SB-13 - for age dating (low priority). [Later whole-rock geochem analysis shows this to be borderline gabbroic diorite- } \\
\text { monzodiorite.] }\end{array}$ \\
\hline
\end{tabular}


CASTLE MOUNTAIN - CARIBOU FAULT SYSTEM FIELD NOTES (referenced to station localities - UTM NAD27 CONUS)

by William A. Fuchs

\begin{tabular}{|c|c|c|c|c|c|c|}
\hline NOTE & SAMPLE & LOCATION & ZN & EASTING & NORTHING & DESCRIPTION \\
\hline B-122 & & & 6 & 440074 & 6859978 & $\begin{array}{l}\text { Elevation: } 3,950 \text { feet } \\
\text { Bedding } 295^{\circ} / 20^{\circ} \mathrm{N} \text {; Talkeetna Formation, agglomerate, just above intrusive contact }\end{array}$ \\
\hline B-123 & & & 6 & 440505 & 6860215 & $\begin{array}{l}\text { Elevation: } 4,280 \text { feet } \\
\text { Bedding } 278^{\circ} / 23^{\circ} \mathrm{N} \text {; Talkeetna Formation, agglomerate }\end{array}$ \\
\hline B-124 & & & 6 & 440086 & 6859689 & $\begin{array}{l}\text { Elevation: } 4,470 \text { feet } \\
\text { Bedding } 061^{\circ} / 28^{\circ} \mathrm{N}\end{array}$ \\
\hline B-125 & & & 6 & 440205 & 6859508 & $\begin{array}{l}\text { Elevation: } 4,460 \text { feet } \\
\text { Bedding } 229^{\circ} / 25^{\circ} \mathrm{S}\end{array}$ \\
\hline B-126 & & & 6 & 440263 & 6859584 & $\begin{array}{l}\text { Elevation: } 4,600 \text { feet } \\
\text { Bedding } 292^{\circ} / 12^{\circ} \mathrm{S}\end{array}$ \\
\hline B-127 & & & 6 & 440219 & 6859617 & $\begin{array}{l}\text { Elevation: } 4,550 \text { feet } \\
\text { Bedding } 356^{\circ} / 42^{\circ} \mathrm{E}\end{array}$ \\
\hline B-128 & & & 6 & 437534 & 6858586 & $\begin{array}{l}\text { Elevation: 4,010 feet } \\
\text { Castle Mountain fault zone. Chickaloon to south, Talkeetna Formation to north; in float. }\end{array}$ \\
\hline B-129 & & & 6 & 437018 & 6858300 & Bedding $055^{\circ} / 82^{\circ} \mathrm{N}$; Matanuska Formation, probably upper Matanuska; very sandstone-rich \\
\hline B-130 & SB-14 & & 6 & 436888 & 6858395 & $\begin{array}{l}\text { Sample SB-14 - Rhyolite-dacite intrusive, [Borderline rhyolite-dacite determined by geochemical whole-rock analysis done } \\
\text { after dissertation completed. See analyses.] }\end{array}$ \\
\hline B-131 & SB-15 & & 6 & 435503 & 6858626 & Sample SB-15 - Float from cliff; felsite intrusive \\
\hline B-132 & & $\begin{array}{l}\text { Boulder Creek } \\
\text { upstream from } \\
\text { cabin }\end{array}$ & 6 & 443904 & 6867823 & $\begin{array}{l}\text { Elevation: } 3,230 \text { feet } \\
\text { Bedding } 156^{\circ} / 62^{\circ} \mathrm{E} \text {; Chinitna Formation. Belemnite found here. Considerable fossilized wood found in stream. Gray } \\
\text { sandstone and siltstone. }\end{array}$ \\
\hline B-133 & & & 6 & 443848 & 6867911 & Fault; vertical displacement, 150 feet \\
\hline B-134 & & & 6 & 443786 & 6868071 & $\begin{array}{l}\text { Unusual fossil in talus (probably close to in-place), like a small crab's leg, preserved in concretion nodule. Sequence appears } \\
\text { to be one-third sandstone, two-thirds siltstone; belemnites fairly common, but few other fossils found; this may be Chinitna } \\
\text { Formation. }\end{array}$ \\
\hline B-135 & & & 6 & 444097 & 6868073 & Bedding $166^{\circ} / 35^{\circ} \mathrm{E}$; Chickaloon Formation, sandstone, plant fossils (wood fragments) seen in stream material. \\
\hline B-136 & & & 6 & 443818 & 6868214 & $\begin{array}{l}\text { Elevation: 3,970 feet } \\
\text { Contact between Chinitna and Chickaloon Formation; } 2 \text { inches soil horizon (clayey) at contact, black siltstone below contact, } \\
\text { boulder-cobble conglomerate above contact. }\end{array}$ \\
\hline B-137 & & & 6 & 443688 & 6868393 & $\begin{array}{l}\text { Elevation: } 4,370 \text { feet } \\
\text { Bedding } 159^{\circ} / 22^{\circ} \mathrm{E} \text {, Chickaloon Formation, sandstone with plant fossils. }\end{array}$ \\
\hline B-138 & SB-16 & & 6 & 443422 & 6868526 & $\begin{array}{l}\text { Tuffaceous(?) sandstone, Chickaloon Formation, very white, several hundred(?) feet thick. This unit has been seen elsewhere, } \\
\text { and can be considered a marker horizon. Sample SB-16, for age dating; coaly fragments; thin coal bed in saddle here. }\end{array}$ \\
\hline B-139 & & & 6 & 443256 & 6868875 & $\begin{array}{l}\text { Elevation: } 4,860 \text { feet } \\
\text { Bedding } 219^{\circ} / 85^{\circ} \mathrm{S} \text {; Chickaloon Formation, tuffaceous sandstone-pebble conglomerate. }\end{array}$ \\
\hline B-140 & & & 6 & 443277 & 6869071 & $\begin{array}{l}\text { Elevation: 4,960 feet } \\
\text { Major fault placing Chickaloon against Tertiary volcanics. Entire Chinitna missing (that is, } \sim 2,000 \text { feet vertical displacement); } \\
\text { trend } 272^{\circ} \text {, fault plane dips to south, normal fault, beds dragged. }\end{array}$ \\
\hline
\end{tabular}


CASTLE MOUNTAIN - CARIBOU FAULT SYSTEM FIELD NOTES (referenced to station localities - UTM NAD27 CONUS)

by William $A$. Fuchs

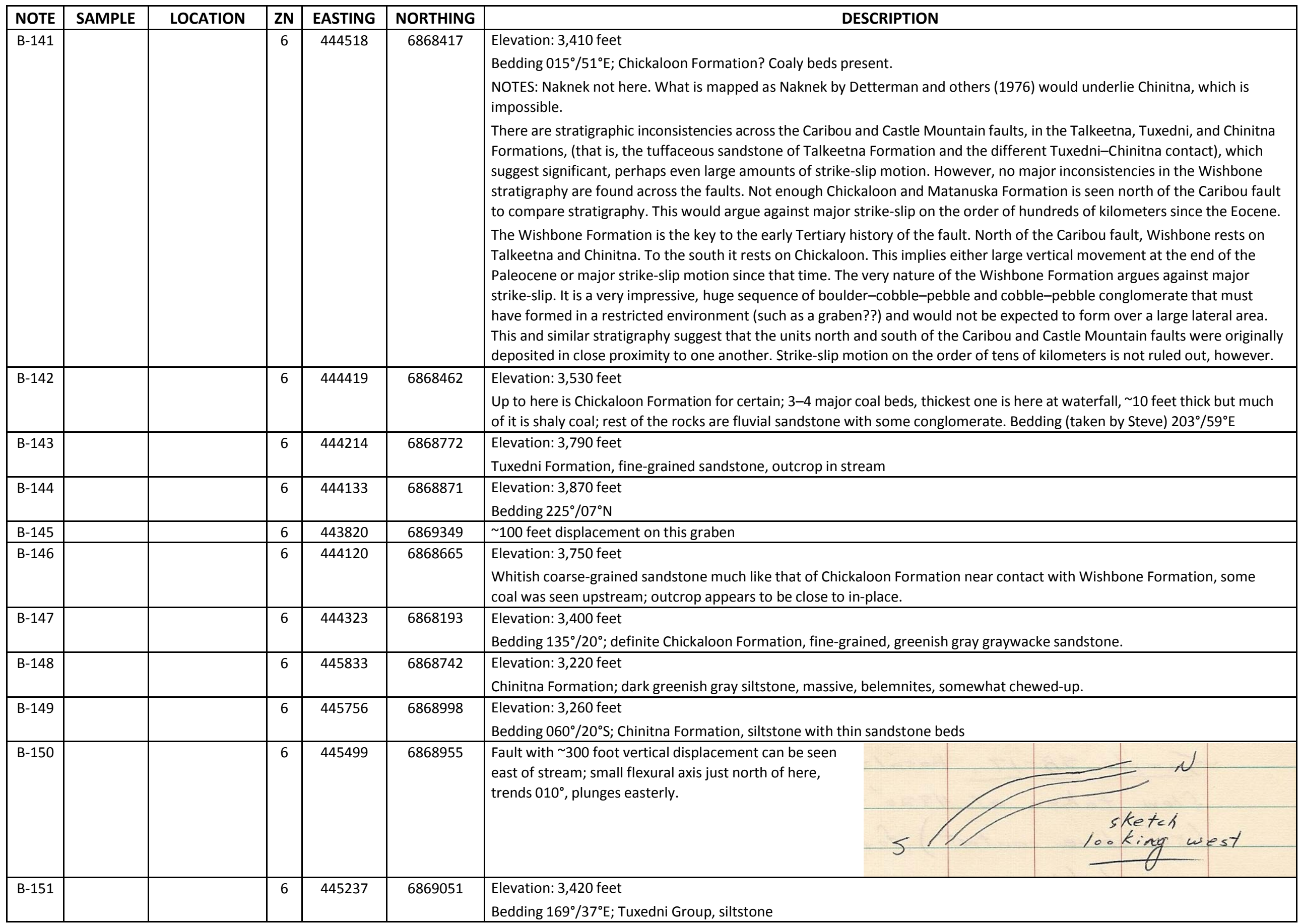


CASTLE MOUNTAIN - CARIBOU FAULT SYSTEM FIELD NOTES (referenced to station localities - UTM NAD27 CONUS)

by William A. Fuchs

\begin{tabular}{|c|c|c|c|c|c|c|}
\hline NOTE & SAMPLE & LOCATION & ZN & EASTING & NORTHING & DESCRIPTION \\
\hline B-152 & & & 6 & 444603 & 6869495 & Bedding $307^{\circ} / 17^{\circ} \mathrm{E}$; Tuxedni Group \\
\hline B-153 & & & 6 & 443716 & 6869899 & $\begin{array}{l}\text { Elevation: 4,080 feet } \\
\text { Tuxedni-Chinitna contact }\end{array}$ \\
\hline B-154 & & & 6 & 444152 & 6870357 & $\begin{array}{l}\text { Elevation: } 4,650 \text { feet } \\
\text { Bedding } 122^{\circ} / 14^{\circ} \mathrm{N} \text {; Chinitna Formation }\end{array}$ \\
\hline B-155 & SB-17 & & 6 & 444161 & 6870472 & $\begin{array}{l}\text { Elevation: } 4,810 \text { feet } \\
\text { Contact of Tertiary volcanics with Chinitna Formation. Sample SB-17, basalt flow taken at 4,860 feet ( } 50 \text { feet above contact), } \\
\text { for age dating. }\end{array}$ \\
\hline B-156 & & & 6 & 444900 & 6868571 & $\begin{array}{l}\text { Elevation: } 3,330 \text { feet } \\
\text { Bedding } 210^{\circ} / 55^{\circ} \mathrm{E} \text {; Chickaloon Formation, fluvial sandstone with abundant wood fossils }\end{array}$ \\
\hline B-157 & & & 6 & 444804 & 6868676 & $\begin{array}{l}\text { Elevation: 3,450 feet } \\
\text { Fault }\end{array}$ \\
\hline B-158 & & & 6 & 445215 & 6868641 & Chickaloon Formation, small outcrop \\
\hline B-159 & & & 6 & 446060 & 6869287 & $\begin{array}{l}\text { Elevation: } 3,840 \text { feet } \\
\text { Bedding } 221^{\circ} / 47^{\circ} \mathrm{E} \text {; Chickaloon Formation }\end{array}$ \\
\hline B-160 & $\begin{array}{l}\text { SB-18 } \\
\text { SB-19 }\end{array}$ & & 6 & 446101 & 6869589 & $\begin{array}{l}\text { Elevation 4,370 ft.; Chickaloon Formation in contact with Tertiary volcanic flow. This Chickaloon is transitional in character to } \\
\text { Wishbone, but more like Chickaloon. Sample SB-18 (critical to tectonic interpretation) small basalt flow, amygdaloidal, } \\
\text { appears to be equivalent of one found on Castle Mountain (see notes for locality K-44); has significance in showing transitional } \\
\text { nature of upper Chickaloon -lower Wishbone (facies equivalents) as well as portending the onset of volcanism; Chickaloon } \\
\text { here is a pebble-cobble conglomerate with abundant black chert and less abundant pebbles of white quartz, Talkeetna Fm. } \\
\text { volcanics, and diorite. Tertiary volcanics consist of massive glassy flow (no basal tuff), blackish brown, weathers pink. Sample } \\
\text { SB-19, for age dating, taken } 10 \text { feet above contact. Existence of coal beds nearby establishes Chickaloon designation. }\end{array}$ \\
\hline B-161 & & & 6 & 446853 & 6869985 & $\begin{array}{l}\text { Elevation: 4,300 feet } \\
\text { White tuffaceous sandstone, Chickaloon or Wishbone(??); contains minor coal }\end{array}$ \\
\hline B-162 & & & 6 & 446811 & 6869905 & $\begin{array}{l}\text { Elevation: } 4,190 \text { feet } \\
\text { Bedding } 132^{\circ} / 14^{\circ} \mathrm{S} \text {; Chickaloon Formation }\end{array}$ \\
\hline B-163 & & & 6 & 447234 & 6869298 & Tuff, Talkeetna? Formation \\
\hline B-164 & $\begin{array}{l}\text { FSB-21 } \\
\text { SB-20 }\end{array}$ & & 6 & 447616 & 6870466 & $\begin{array}{l}\text { Elevation: 3,400 feet } \\
\text { Bedding } 124^{\circ} / 43^{\circ} \mathrm{N} \text {, taken on north-side of stream; Matanuska Formation, abundant Inoceramus, Sample FSB-21 (Steve's } \\
\text { possession): One belemnite, worm burrows. Sample SB-20 (for Eckdale): Biotitic graywacke in places, sandstone is fine- to } \\
\text { medium-grained, greenish gray Matanuska-Chickaloon contact is grandly exposed at waterfall where a fine-grained gray- } \\
\text { wacke directly underlies a boulder-cobble-pebble conglomerate } 100 \text { feet thick (Chickaloon Formation). Above that is } \\
\text { typical whitish, coarse-grained Chickaloon sandstone with coal beds (upper Chickaloon Formation). }\end{array}$ \\
\hline B-165 & & & 6 & 447327 & 6870655 & $\begin{array}{l}\text { Elevation: } 3,970 \text { feet } \\
\text { Bedding } 026^{\circ} / 23^{\circ} \mathrm{E} \text {; Chickaloon Formation, } 10 \text { foot section of bituminous coal interlayered with shale. }\end{array}$ \\
\hline B-166 & & & 6 & 447314 & 6870706 & $\begin{array}{l}\text { Elevation: } 4,060 \text { feet } \\
\text { Fault cutting off coal beds, slickenside surface; attitude } 254^{\circ} / 64^{\circ} \mathrm{S}\end{array}$ \\
\hline
\end{tabular}


CASTLE MOUNTAIN - CARIBOU FAULT SYSTEM FIELD NOTES (referenced to station localities - UTM NAD27 CONUS)

by William A. Fuchs

\begin{tabular}{|c|c|c|c|c|c|c|}
\hline NOTE & SAMPLE & LOCATION & ZN & EASTING & NORTHING & DESCRIPTION \\
\hline B-167 & & & 6 & 444396 & 6866981 & $\begin{array}{l}\text { Elevation: } 2,990 \text { feet } \\
\text { Bedding } 047^{\circ} / 27^{\circ} \mathrm{N} \text {; Matanuska Formation, black siltstone, Inoceromus present; small cataclastic fault zone just downstream } \\
\text { trends } 0^{\circ} ; \text { much felsic intrusive in stream float. }\end{array}$ \\
\hline B-168 & & & 6 & 444439 & 6866716 & $\begin{array}{l}\text { Elevation: } 3,730 \text { feet } \\
\text { Talkeetna Formation }\end{array}$ \\
\hline B-169 & & & 6 & 444364 & 6866876 & $\begin{array}{l}\text { Elevation: } 3,440 \text { feet } \\
\text { Possible position of fault, cataclasite in float. }\end{array}$ \\
\hline B-170 & & & 6 & 444538 & 6866915 & Offset moraine? Has definite landslide features (ponding) \\
\hline B-171 & & & 6 & 444628 & 6866513 & $\begin{array}{l}\text { Elevation: } 3,940 \text { feet } \\
\text { Projection of dike, trend }\end{array}$ \\
\hline B172 & & & 6 & 443294 & 6868439 & Displacement on fault $\sim 100$ feet \\
\hline B-173 & & & 6 & 443251 & 6868566 & Displacement on fault $\sim 50$ feet \\
\hline B-174 & & & 6 & 442951 & 6868336 & $\begin{array}{l}\text { Elevation: } 4,550 \text { feet } \\
\text { Chinitna-Chickaloon contact }\end{array}$ \\
\hline B-175 & & & 6 & 442646 & 6868269 & $\begin{array}{l}\text { Elevation: } 4,700 \text { feet } \\
\text { Bedding } 172^{\circ} / 31^{\circ} \mathrm{E} \text {; Chickaloon Formation }\end{array}$ \\
\hline B-176 & & & 6 & 442534 & 6868030 & $\begin{array}{l}\text { Elevation: } 4,470 \text { feet } \\
\text { Chinitna-Chickaloon contact }\end{array}$ \\
\hline B-177 & & & 6 & 442946 & 6866215 & $\begin{array}{l}\text { Elevation: 3,210 feet } \\
\text { Fault zone - mostly Matanuska in zone; cataclastic zone goes from here up to elevation 3,330 feet }\end{array}$ \\
\hline B-178 & & & 6 & 443268 & 6865452 & Altered highly pyritic felsic intrusive. \\
\hline B-179 & & & 6 & 443853 & 6866569 & $\begin{array}{l}\text { Elevation: 3,470 feet } \\
\text { Fault zone }\end{array}$ \\
\hline B-180 & & & 6 & 446191 & 6867883 & $\begin{array}{l}\text { Elevation: } 3,390 \text { feet } \\
\text { Talkeetna Formation; bedding ( } 100 \text { feet upstream from here on hillside) } 074^{\circ} / 43^{\circ} \mathrm{N} \text {; well-bedded volcanics; unusual to find } \\
\text { measureable bedding in this block. }\end{array}$ \\
\hline B-181 & SB-21 & & 6 & 445882 & 6866010 & $\begin{array}{l}\text { Elevation: 4,710 feet } \\
\text { Sample SB-21 - Rhyolite intrusive [Determined to be rhyolite by geochemical whole-rock analysis done after dissertation } \\
\text { completed. See analyses.] }\end{array}$ \\
\hline B-182 & SB-22 & & 6 & 445647 & 6865357 & $\begin{array}{l}\text { Elevation: 5,210 feet } \\
\text { Sample SB-22 - (two samples not in bags) Porphyry phase of dacite intrusive. *For age dating K-Ar on hornblende separate } \\
\text { [Determined to be dacite by geochemical whole-rock analysis done after dissertation completed. See analyses.] }\end{array}$ \\
\hline B-183 & & & 6 & 448646 & 6869815 & $\begin{array}{l}\text { Looks very much like glassy basal Tertiary volcanic. Above here definitely Tertiary volcanic, below I don't know (could be } \\
\text { same or Talkeetna Formation). Immediately below glassy stuff it looks vesicular and like Tertiary volcanic. }\end{array}$ \\
\hline B-184 & & & 6 & 450107 & 6871061 & Could easily be Talkeetna Formation, but may also be Tertiary volcanic. \\
\hline B-185 & & & 6 & 449047 & 6867453 & $\begin{array}{l}\text { Mapped by Detterman and others (1976) as Tertiary mafic intrusive. Not so. Intrusive porphyry, which is probably a volcanic } \\
\text { pipe of the Talkeetna Formation. }\end{array}$ \\
\hline B-186 & SB-23 & & 6 & 447609 & 6868636 & Sample SB-23 - Age dating (whole rock?) would resolve whether this is Talkeetna or Tertiary volcanics - pink flow breccia. \\
\hline B-187 & & & 6 & 448531 & 6868838 & $\begin{array}{l}\text { Elevation: } 3,870 \text { feet } \\
\text { No doubt about it - Caribou fault zone in exposure }\end{array}$ \\
\hline
\end{tabular}


CASTLE MOUNTAIN - CARIBOU FAULT SYSTEM FIELD NOTES (referenced to station localities - UTM NAD27 CONUS)

by William A. Fuchs

\begin{tabular}{|c|c|c|c|c|c|c|}
\hline NOTE & SAMPLE & LOCATION & ZN & EASTING & NORTHING & DESCRIPTION \\
\hline B-188 & & & 6 & 449051 & 6868635 & $\begin{array}{l}\text { Elevation: } 4,030 \text { feet } \\
\text { Fault zone, trends } 126^{\circ} \text {, contains felsic (?) intrusive }\end{array}$ \\
\hline B-189 & & & 6 & 449211 & 6868794 & $\begin{array}{l}\text { Elevation: } 4,240 \text { feet } \\
\text { Fault zone, probably large fault, trending } 119^{\circ} \text {, occupied by gabbro dike }\end{array}$ \\
\hline B-191 & \begin{tabular}{l|l} 
SB-11 \\
SB-11alt.
\end{tabular} & & 6 & 440132 & 6858164 & $\begin{array}{l}\text { Sample SB-11 and SB-11 alt. - Rhyolite intrusive; offset stock? - collected via Amoco's helicopter. [Determined to be rhyolite } \\
\text { by geochemical whole-rock analysis done after dissertation completed. See analyses.] }\end{array}$ \\
\hline
\end{tabular}

\section{CH-1 through CH-5 completed during summer 1978 along west side Chickaloon River to 16 Mile}

\begin{tabular}{|c|c|c|c|c|c|c|}
\hline $\mathrm{CH}-1$ & & $\begin{array}{l}\text { West side Chick- } \\
\text { aloon River }\end{array}$ & 6 & 430416 & 6867763 & $\begin{array}{l}\text { Elevation: } 2,240 \text { feet } \\
\text { Outcrop begins at } 2,210 \text { feet. Bedding } 265^{\circ} / 24^{\circ} \mathrm{N} \text {; Talkeetna Formation? Bedded tuffaceous sandstone; significant shearing } \\
\text { seen where outcrop begins. }\end{array}$ \\
\hline $\mathrm{CH}-2$ & & $\begin{array}{l}\text { West side Chick- } \\
\text { aloon River }\end{array}$ & 6 & 428985 & 6868161 & $\begin{array}{l}\text { Elevation: 3,140 feet } \\
\text { Diorite or monzonite intrusive, medium-grained, fresh, two feldspars present (one pink). From } \mathrm{CH}-1 \text { to here has been } \\
\text { Talkeetna Formation. Just upstream from } \mathrm{CH}-1 \text { is a beautiful, thick section of well-bedded tuffaceous sandstone, same as } \\
\text { found up } 9 \text { Mile Creek, very distinctive (bedding, white color, mottled appearance), at least several hundred feet thick, } \\
\text { farther upstream are more flows, and the rocks are more messed up, actually cataclastic in places. }\end{array}$ \\
\hline $\mathrm{CH}-3$ & AG-1 & $\begin{array}{l}\text { West side Chick- } \\
\text { aloon River }\end{array}$ & 6 & 428755 & 6868131 & $\begin{array}{l}\text { Elevation: } 3,250 \text { feet } \\
\text { Sample AG-1, for age dating of pluton. Diorite or monzonite, fresh intrusive, medium-grained, some calcite veining in adja- } \\
\text { cent rock, but almost none in this sample. }\end{array}$ \\
\hline $\mathrm{CH}-4$ & AG-2 & $\begin{array}{l}\text { West side Chick- } \\
\text { aloon River }\end{array}$ & 6 & 431423 & 6871100 & $\begin{array}{l}\text { Elevation: } 2,240 \text { feet } \\
\text { Lowest outcrop on stream. Fresh intrusive, similar to that of sample AG-1, but finer grained and much less calcite veining. } \\
\text { Sample AG-2, for age dating. }\end{array}$ \\
\hline $\mathrm{CH}-5$ & & $\begin{array}{l}\text { West side Chick- } \\
\text { aloon River }\end{array}$ & 6 & 430657 & 6871155 & $\begin{array}{l}\text { Everything from } \mathrm{CH}-4 \text { to here is fresh intrusive; all of the float in the streambed is intrusive, some of it with limonitic alter- } \\
\text { ation. }\end{array}$ \\
\hline
\end{tabular}

79-1 through 79-86 summer 1979

\begin{tabular}{|c|c|c|c|c|c|c|}
\hline $79-1$ & F79-1 & $\begin{array}{l}\text { Anchorage D2 } \\
\text { Quadrangle }\end{array}$ & 6 & 463968 & 6852125 & $\begin{array}{l}\text { Talkeetna Formation; fractured and messed-up agglomerate or lapilli tuff. Sample F79-1 for age dating; locality is 300 feet } \\
\text { south of bridge crossing Caribou Creek; first good outcrop on west side of road. }\end{array}$ \\
\hline $79-2$ & F79-2 & $\begin{array}{l}\text { Lion Head } \\
\text { (above RCA } \\
\text { tower) }\end{array}$ & 6 & 464841 & 6850408 & $\begin{array}{l}\text { Elevation: } 2,370 \text { feet } \\
\text { Location out of map area. Sample F79-2 -Rhyolite from volcanic plug (just like all the others), very well displayed cooling } \\
\text { joints, rock fine-grained, porphyritic; sampled for age dating and } \mathrm{Rb}, \mathrm{Sr}, \mathrm{Ba} \text { analysis. [Determined to be rhyolite by } \\
\text { geochemical whole-rock analysis done after dissertation completed. See analyses.] }\end{array}$ \\
\hline $79-3$ & F79-3 & West of F79-2 & 6 & 464637 & 6850373 & $\begin{array}{l}\text { Elevation: 2,460 feet } \\
\text { Location out of map area. Location out of map area. Rhyolite; locality should actually be plotted halfway between F79-2 and } \\
\text { F79-4. [Determined to be rhyolite by geochemical whole-rock analysis done after dissertation completed. See analyses.] }\end{array}$ \\
\hline $79-4$ & F79-4 & West of F79-3 & 6 & 464432 & 6850323 & $\begin{array}{l}\text { Elevation: } 2,430 \text { feet } \\
\text { Location out of map area. Sample F79-4 - Rhyolite. [Determined to be rhyolite by geochemical whole-rock analysis done } \\
\text { after dissertation completed. See analyses.] } \\
\text { 6/24/79: Observed geology up Moose Creek; saw Tsadaka-Chickaloon contact. Poorly consolidated boulder-cobble-pebble } \\
\text { conglomerate unconformably overlies black siltstone; contains abundant granitic material. Contact can only be observed on } \\
\text { west side of creek from east side. No dikes observed in Tsadaka Formation. }\end{array}$ \\
\hline $79-5$ & & & 6 & 435098 & 6860741 & $\begin{array}{l}\text { Elevation: 3,010 feet } \\
\text { Outcrop of medium-grained graywacke; bedding not apparent, moderately fractured; could be Matanuska Formation or } \\
\text { Tuxedni Group. }\end{array}$ \\
\hline
\end{tabular}


CASTLE MOUNTAIN - CARIBOU FAULT SYSTEM FIELD NOTES (referenced to station localities - UTM NAD27 CONUS)

by William $A$. Fuchs

\begin{tabular}{|c|c|c|c|c|c|c|}
\hline NOTE & SAMPLE & LOCATION & ZN & EASTING & NORTHING & DESCRIPTION \\
\hline $79-6$ & & & 6 & 434997 & 6860726 & $\begin{array}{l}\text { Elevation: } 3,160 \text { feet } \\
\text { Bedding in graywacke } 351^{\circ} / 28^{\circ} \mathrm{E} \text {; formation? }\end{array}$ \\
\hline $79-7$ & & & 6 & 435070 & 6860759 & $\begin{array}{l}\text { Elevation: } 3,060 \text { feet } \\
25 \text { feet upstream from here is Talkeetna Formation; purple andesite porphyry }\end{array}$ \\
\hline $79-8$ & & & 6 & 434829 & 6860970 & $\begin{array}{l}\text { Elevation: 3,310 feet } \\
\text { Fault zone in Talkeetna Formation, } 10 \text { feet wide, chloritic cataclasite with fault breccia; trends } 077^{\circ} \text { (heavy topographic } \\
\text { influence); dip couldn't be determined, but is moderate. } \\
\text { Upstream from } 79-8 \text { to an elevation of } 3,470 \text { feet, it is apparent that the formation to the south is Tuxedni Group. Evidence: } \\
\text { fossil wood, abundant belemnites, several ammonite fragments in stream bed; overall appearance of section similar to that } \\
\text { in vicinity of the area southwest of B-116. }\end{array}$ \\
\hline $79-9$ & & & 6 & 434780 & 6860426 & $\begin{array}{l}\text { Elevation: } 3,670 \text { feet } \\
\text { Matanuska? Formation; unusual bed } 4 \text { feet thick with mottled appearance; white spots appear to be tuffaceous material } \\
\text { (dateable??); everything downslope has been medium-grained graywacke, few fossils, some woody fragments; above this } \\
\text { point some silty beds occur. } \\
\text { Bedding: } 40^{\circ} / 16^{\circ} \mathrm{W}, 01^{\circ} / 10^{\circ} \mathrm{W}, 56^{\circ} / 15^{\circ} \mathrm{W}, 10^{\circ} / 07^{\circ} \mathrm{W} \text { (average of four readings } 027^{\circ} / 12^{\circ} \mathrm{W} \text { ) }\end{array}$ \\
\hline $79-10$ & & & 6 & 434408 & 6860667 & $\begin{array}{l}\text { Elevation: } 4,490 \text { feet } \\
\text { Diabase dike, trend } 055^{\circ}\end{array}$ \\
\hline $79-11$ & & & 6 & 435558 & 6861029 & $\begin{array}{l}\text { Elevation: } 2,990 \text { feet } \\
\text { Diabase dike, } 100 \text { feet thick, attitude } 130 \% \text { vertical, intruding latite; latite includes abundant xenoliths of Talkeetna Forma- } \\
\text { tion, flow breccia; may be near edge of latite dome. }\end{array}$ \\
\hline $79-12$ & F79-12 & & 6 & 435554 & 6861116 & $\begin{array}{l}\text { Elevation: 3,030 feet } \\
\text { Mineralization in Talkeetna Formation, flow breccia and flows? Pyritic, highly oxidized, argillic alteration; on west side of } \\
\text { stream; has been examined by others (evidence of rock-hammer blows); color not right for copper; what appears to be a } \\
\text { porphyry intrusive occurs along stream. Sample F79-12 for thin section and comparison with quartz-eye porphyry (albite } \\
\text { granite porphyry), which it somewhat resembles. The sample taken is quite fresh - light gray matrix with phenocrysts of feld- } \\
\text { spar and quartz. Sample also resembles that which was called Talkeetna Formation (although USGS mapped it as unit Tim) in } \\
\text { Section 3, T21N, R8E. F79-12 may be the mineralizer here. }\end{array}$ \\
\hline $79-13$ & F79-13 & & 6 & 435554 & 6861190 & $\begin{array}{l}\text { Elevation: 3,060 feet } \\
\text { Sample F79-13 - pyritic. Talkeetna(?) Formation; highly altered. }\end{array}$ \\
\hline $79-14$ & F79-14 & & 6 & 435727 & 6861282 & $\begin{array}{l}\text { Elevation: } 3,220 \text { feet } \\
\text { Sample F79-14 - pyritically altered Talkeetna(?) Formation }\end{array}$ \\
\hline $79-15$ & & & 6 & 434966 & 6861087 & $\begin{array}{l}\text { Elevation: } 3,800 \text { feet } \\
\text { Altered Talkeetna Formation, bleached and pyritic; dike nearby (to east) has trend of } 326^{\circ} ; \text { Talkeetna Formation here has a } \\
\text { great unconformity between it and Tuxedni Group. Talkeetna in fact seems to be a submerged mountain. This could represent } \\
\text { a Jurassic volcanic center. Angular unconformity between Tuxedni Group and Matanuska Formation seen across valley to } \\
\text { south. Fault across valley has displacement of } \sim 100 \text { feet, high-angle, south side down. }\end{array}$ \\
\hline $79-16$ & F19-16 & & 6 & 434855 & 6861473 & $\begin{array}{l}\text { Sample F79-16 - (two bags) for age dating. Talkeetna Formation, andesite porphyry flow breccia, collected near what is } \\
\text { thought to be top of Talkeetna section. Similar to quartz-eye porphyry, but I believe it is Talkeetna Formation; may be from a } \\
\text { vent of a volcano that fed Talkeetna Formation. }\end{array}$ \\
\hline $79-17$ & F79-17 & & 6 & 434727 & 6861674 & $\begin{array}{l}\text { Tuxedni contact just below here. Bedded sandstone, bedding, } 170^{\circ} / 25^{\circ} \mathrm{S} \text { (actually very well cross-bedded, with definite evi- } \\
\text { dence beds are right side up). Sample F } 79-17 \text { - of sandstone for reference; unit is } \sim 25 \text { feet thick; below this is a greenish } \\
\text { chloritic, tuffaceous unit similar to that seen elsewhere. }\end{array}$ \\
\hline
\end{tabular}


CASTLE MOUNTAIN - CARIBOU FAULT SYSTEM FIELD NOTES (referenced to station localities - UTM NAD27 CONUS)

by William $A$. Fuchs

\begin{tabular}{|c|c|c|c|c|c|c|}
\hline NOTE & SAMPLE & LOCATION & ZN & EASTING & NORTHING & DESCRIPTION \\
\hline $79-18$ & & & 6 & 434661 & 6861686 & $\begin{array}{l}\text { Bedding in highly fossiliferous Tuxedni Group. Bedding } 029^{\circ} / 23^{\circ} \mathrm{W} \text {; clams, brachiopods, gastropods, belemnites, fossil wood } \\
\text { (similar to locality B-103?); also fossiliferous upslope from here. } \\
\text { Measured section - Tuxedni Group; from bottom to top } \\
\text { UNIT } 1 \text { - } 250 \text { feet true thickness (estimated). Sandstone, medium-grained, massive to medium-bedded ( } 8 \text { inches), feldspathic, } \\
\text { green-gray (unweathered), slightly orange-brown (weathered). Bedding } 140^{\circ} / 17^{\circ} \mathrm{S} \text {. Not many fossils, wood fragments. } \\
\text { UNIT } 2-1,250 \text { ? feet thick. Bedding } 136^{\circ} / 24^{\circ} \mathrm{S} \text {. Bearing } 265^{\circ} \text {, slope } 27^{\circ} .{ }^{*} 50 \mathrm{feet}+50 \mathrm{feet}+50 \mathrm{feet;} \text { predominantly siltstone, } \\
\text { brown to maroon-gray (weathered color), lesser sandstone and shale. } \\
\text { UNIT } 3 \text { - } 60 \text { feet (not true thickness); green-gray feldspathic sandstone, medium-grained. I'm calling this Tuxedni Group, but } \\
\text { could be Matanuska Formation; attitude similar to below. } \\
\text { Matanuska Formation - black argillite. } \\
\text { Note: I have not noted fossil content in this section because access to the rocks was difficult. Not many fossils were seen, } \\
\text { and section appears to be significantly less fossiliferous than that to the north of the Caribou fault. } \\
\text { *Total section thickness = } 370 \text { meters. (Unit } 3 \text { assumed to be Matanuska Formation.) }\end{array}$ \\
\hline $79-19$ & H79-19 & & 6 & 435716 & 6860332 & $\begin{array}{l}\text { Elevation: 3,170 feet } \\
\text { Talkeetna Formation in small outcrop. Volcaniclastic sandstone and minor tuff. Sample H79-19 (Donna's sample) }\end{array}$ \\
\hline $79-20$ & F79-20 & & 6 & 435960 & 6860609 & $\begin{array}{l}\text { Elevation: 3,670 feet } \\
\text { Rhyolite, Sample F79-20 - for Rb, Sr, Ba analysis, slightly weathered. [Determined to be rhyolite by geochemical whole-rock } \\
\text { analysis done after dissertation completed. See analyses.] }\end{array}$ \\
\hline $79-21$ & & & 6 & 436097 & 6860670 & $\begin{array}{l}\text { Elevation: } 3,830 \text { feet } \\
\text { Diabase dike, minimum } 5 \text { feet wide, } 090^{\circ} / \text { vertical }\end{array}$ \\
\hline $79-22$ & F79-22 & & 6 & 436353 & 6860829 & $\begin{array}{l}\text { Elevation: 4,080 feet } \\
\text { Rhyolite, Sample F79-22 - for Rb, Sr, Ba analysis. [Determined to be rhyolite by geochemical whole-rock analysis done after } \\
\text { dissertation completed. See analyses.] }\end{array}$ \\
\hline $79-23$ & F79-23 & & 6 & 436457 & 6861123 & $\begin{array}{l}\text { Elevation: 4,530 feet } \\
\text { Bearing on rhyolite peak Anthracite Ridge }=170^{\circ} \text {. Rhyolite, Sample F79-23 - for Rb, Sr, Ba. [Determined to be rhyolite by } \\
\text { geo- chemical whole-rock analysis done after dissertation completed. See analyses.] } \\
\text { Coming down the slope: Talkeetna/rhyolite contact 4,240 feet; rhyolite/Talkeetna contact 4,060 feet; Talkeetna/rhyolite } \\
\text { contact, 3,270 feet. }\end{array}$ \\
\hline $79-24$ & F79-24 & $\begin{array}{l}\text { West side, An- } \\
\text { thracite Ridge }\end{array}$ & 6 & 435256 & 6858578 & $\begin{array}{l}\text { Sample F79-24, dacite for } \mathrm{Rb}, \mathrm{Sr}, \mathrm{Ba} \text { analysis. [Determined dacite by geochemical whole-rock analysis done after dissertation } \\
\text { completed. See analyses.] }\end{array}$ \\
\hline $79-25$ & F79-25 & & 6 & 438260 & 6863667 & $\begin{array}{l}\text { Elevation: 3,910 feet } \\
\text { Talkeetna andesite porphyry, flow? Purple. Lies just below altered Talkeetna Formation and its contact with Tuxedni Group } \\
\text { (below Boulder Creek fault). Sample F79-25 for age dating (fission-track) represents youngest Talkeetna Formation? }\end{array}$ \\
\hline $79-26$ & & & 6 & 433474 & 6859616 & $\begin{array}{l}\text { Elevation: } 3,480 \text { feet } \\
\text { Bedding } 196^{\circ} / 09^{\circ} \mathrm{W} \text {, Tertiary volcanics. Below this point are basaltic flow and diabase feeders; above are flow breccias and } \\
\text { flows. }\end{array}$ \\
\hline $79-27$ & F79-27 & & 6 & 433576 & 6859631 & $\begin{array}{l}\text { Elevation: 3,320 feet } \\
\text { Sample F79-27, Tertiary volcanics; porphyritic andesite flow, probably within } 50 \text { feet of base of volcanic sequence, for age } \\
\text { dating. }\end{array}$ \\
\hline $79-28$ & & & 6 & 434284 & 6859693 & Matanuska Formation, argillite - Castle Mountain fault must be south of here. No evidence of Wishbone Formation downslope. \\
\hline $79-29$ & & & 6 & 433489 & 6859791 & Possible double fault scarp on Castle Mountain fault; very pronounced. \\
\hline
\end{tabular}


CASTLE MOUNTAIN - CARIBOU FAULT SYSTEM FIELD NOTES (referenced to station localities - UTM NAD27 CONUS)

by William A. Fuchs

\begin{tabular}{|c|c|c|c|c|c|c|}
\hline NOTE & SAMPLE & LOCATION & ZN & EASTING & NORTHING & DESCRIPTION \\
\hline $79-30$ & & & 6 & 434003 & 6859768 & $\begin{array}{l}\text { Elevation: } 2,810 \text { feet } \\
\text { Very chewed up Matanuska Formation. Inferred that Castle Mountain splay fault lies just to the south. }\end{array}$ \\
\hline $79-31 \mathrm{~A}$ & & & 6 & 433199 & 6860823 & $\begin{array}{l}\text { Elevation: } 3,550 \text { feet } \\
\text { Bedding } 295^{\circ} / 19^{\circ} \mathrm{N} \text { in Matanuska graywacke }\end{array}$ \\
\hline $79-32 A$ & & & 6 & 433060 & 6861218 & $\begin{array}{l}\text { Elevation: } 4,170 \text { feet } \\
\text { Bedding: } 224^{\circ} / 17^{\circ} \mathrm{W} ; 261^{\circ} / 15^{\circ} \mathrm{N} \text { (slightly upslope); average } 233^{\circ} / 16^{\circ} \mathrm{N} \text {. Matanuska Forma- } \\
\text { tion; argillite with some sandstone and concretionary beds. } \\
\text { Note: Attitude of } 79-32 \text { is not quite as steep as block as a whole is assumed to be. Reason: } \\
\text { Later undulatory folding, which should steepen dips in some places and decrease them in others. Example: Superimpose a } \\
\text { sine wave on a plane. }\end{array}$ \\
\hline $79-31 B$ & & & 6 & 434577 & 6864071 & $\begin{array}{l}\text { Bedding } 056^{\circ} / 71^{\circ} \mathrm{W} \text { in Matanuska Formation. Whole section below here is certainly same as across valley - very } \\
\text { unfossiliferous, shaly siltstones with interspersed sandstone beds. }\end{array}$ \\
\hline $79-32 B$ & F79-32 & & 6 & 434314 & 6863566 & Inoceramus fossils; Ammonite (Sample F79-32) just south of here. \\
\hline $79-33$ & & & 6 & 434130 & 6863232 & $\begin{array}{l}\text { Bedding } 218^{\circ} / 46^{\circ} \mathrm{W} \\
\text { Note: That which was mapped as Chinitna last year is now assumed to be Matanuska Formation. Reason: Rock on ridge north } \\
\text { of } 79-31 \text { is same as across valley, and lies stratigraphically above rock of } 79-32 \text { which is certainly Matanuska Formation. No } \\
\text { noticeable unconformity observed. }\end{array}$ \\
\hline $79-34$ & D79-34 & & 6 & 434884 & 6864323 & Sample D79-34 - Trace fossils in Matanuska Formation. \\
\hline $79-35$ & & & 6 & 435096 & 6864981 & $\begin{array}{l}\text { Altered tuff. Mapped as limestone by Detterman and others (1976) but it is Talkeetna tuff similar to Sample B-6. The fizzing } \\
\text { with acid is due to calcite veining. }\end{array}$ \\
\hline $79-36$ & & & 6 & 435237 & 6863709 & $\begin{array}{l}\text { Elevation: } 4,270 \text { feet } \\
\text { Bedding } 042^{\circ} / 45^{\circ} \mathrm{N} \text {; similar to splay block as a whole? Matanuska Formation. }\end{array}$ \\
\hline $79-37$ & & & 6 & 435074 & 6863541 & Matanuska Formation; pebble conglomerate unit 3 feet thick, discontinuous in thickness. \\
\hline $79-38$ & & & 6 & 434852 & 6863242 & Matanuska Formation. Bedding $047^{\circ} / 57^{\circ} \mathrm{W}$. \\
\hline $79-39$ & & & 6 & 435467 & 6865838 & Bedding $059^{\circ} / 26^{\circ} \mathrm{W} ;$ Talkeetna Formation, tuffaceous sandstone. \\
\hline $79-40$ & F79-40 & & 6 & 435882 & 6865553 & $\begin{array}{l}\text { Sample F79-40, agglomerate, Talkeetna Formation, for age dating (two bags), from 50 feet below Tuxedni-Talkeetna contact. } \\
\text { Notes concerning the Chinitna versus Matanuska Formation argument: In USGS Bulletin 776, Martin (1913) describes fossil } \\
\text { evidence of Chinitna north of Boulder Creek. The main criterion appears to be the existence of Cardiocerus (Callovian, Europe). } \\
\text { Of the three sampling localities, in each case (based on mapping by this author), it appears as if Tuxedni may have been } \\
\text { sampled rather than Chinitna Formation. Either Cardiocerus may have been misidentified or could Cardiocerus (an ammonite) } \\
\text { also have existed in Middle Jurassic (versus Upper Jurassic). (Is our time scale so well defined? Especially when correlating an } \\
\text { Alaskan species with a European species.) }\end{array}$ \\
\hline $79-41$ & F79-41 & & 6 & 434402 & 6861016 & $\begin{array}{l}\text { Pebble conglomerate, } 2 \text { feet thick in Matanuska Formation. Probably correlates with that of 79-37. Potential 3-point } \\
\text { problem. Sample F79-41 for reference. }\end{array}$ \\
\hline $79-42$ & & & 6 & 433402 & 6861783 & $\begin{array}{l}\text { Elevation: } 4,880 \text { feet } \\
\text { Bedding } 175^{\circ} / 32^{\circ} \mathrm{W} \text {; Matanuska Formation, medium-grained greenish graywacke, medium bedded. }\end{array}$ \\
\hline $79-43$ & & & 6 & 432840 & 6859740 & $\begin{array}{l}\text { Elevation: 3,950 feet } \\
\text { Tertiary volcanic; glassy flow probably correlative with basal flows north of Boulder Creek, but here there are hundreds of } \\
\text { feet of underlying volcanics. }\end{array}$ \\
\hline
\end{tabular}


CASTLE MOUNTAIN - CARIBOU FAULT SYSTEM FIELD NOTES (referenced to station localities - UTM NAD27 CONUS)

by William A. Fuchs

\begin{tabular}{|c|c|c|c|c|c|c|c|c|}
\hline NOTE & SAMPLE & LOCATION & ZN & EASTING & NORTHING & \multicolumn{3}{|c|}{ DESCRIPTION } \\
\hline $79-44$ & F79-44 & & 6 & 432527 & 6860078 & \multicolumn{3}{|c|}{$\begin{array}{l}\text { Elevation: 4,160 feet } \\
\text { Castle Mountain splay fault zone. Must be at least } 200 \text { feet wide (probably closer to } 300 \text { feet wide) in this saddle. Sample } \\
\text { F79-44 (two bags) - calcite from fault zone for thermoluminescence dating. Most calcite collected from colluvial float, but } \\
\text { some in place, and all of it clearly came out of fault zone. The calcite occurs in shear fractures and in fault breccia. The source } \\
\text { of all this calcite is the Tertiary volcanics, but it also occurs to a lesser extent in Matanuska Formation in the vicinity of 79-45 } \\
\text { slickenside measurements. }\end{array}$} \\
\hline $79-45$ & F79-45 & & 6 & 436131 & 6857967 & \multicolumn{3}{|c|}{$\begin{array}{l}\text { Elevation: 3,320 feet } \\
\text { Sample F79-45 - Dacite for Rb, Sr, Ba. [Determined to be dacite by geochemical whole-rock analysis done after dissertation } \\
\text { completed. See analyses.] }\end{array}$} \\
\hline \multirow[t]{13}{*}{$79-45 x$} & & & 6 & 432762 & 6860239 & \multicolumn{3}{|c|}{$\begin{array}{l}\text { Strain analysis on Castle Mountain Splay fault slickenside measurements. } \\
\qquad(S=\text { slickenside lineation; } L L=\text { left lateral; } R L=\text { right lateral) }\end{array}$} \\
\hline & & & & & & $168^{\circ} / 71^{\circ} \mathrm{W}-$ Omit & $137^{\circ} / 86^{\circ} \mathrm{S}-\mathrm{S}=20^{\circ} \mathrm{S}$ & $221^{\circ} /$ vert. $-\mathrm{S}=15^{\circ} \mathrm{S}$ \\
\hline & & & & & & $109^{\circ} / 58^{\circ} \mathrm{S}-\mathrm{S}=78^{\circ} \mathrm{E}$ & $156^{\circ} / 81^{\circ} \mathrm{W}-\mathrm{S}=17^{\circ} \mathrm{E}$ & $135^{\circ} / 72^{\circ} \mathrm{E}-\mathrm{S}=12^{\circ} \mathrm{S}$ \\
\hline & & & & & & $005^{\circ} / 80^{\circ} \mathrm{W}-\mathrm{S}=13^{\circ} \mathrm{S}$ & $200^{\circ} / 76^{\circ} \mathrm{W}-\mathrm{S}=42^{\circ} \mathrm{S}$ & $071^{\circ} / 68^{\circ} \mathrm{N}-\mathrm{S}=19^{\circ} \mathrm{W}$ \\
\hline & & & & & & $132^{\circ} / 84^{\circ} \mathrm{W}-\mathrm{S}=85^{\circ} \mathrm{S}$ & $338^{\circ} / 35^{\circ} \mathrm{E}-\mathrm{S}=90^{\circ}$ ?LL? & $138^{\circ} / 80^{\circ} \mathrm{S}-\mathrm{S}=12^{\circ} \mathrm{S}$ \\
\hline & & & & & & $103^{\circ} / 81^{\circ} \mathrm{N}-\mathrm{S}=0^{\circ}$ & $132^{\circ} / 81^{\circ} \mathrm{N}-\mathrm{S}=34^{\circ} \mathrm{S}$ & $167^{\circ} / 83^{\circ} \mathrm{W}-\mathrm{S}=0^{\circ}$ \\
\hline & & & & & & $180^{\circ} / 80^{\circ} \mathrm{W}-\mathrm{S}=0^{\circ}$ & $183^{\circ} / 80^{\circ} \mathrm{W}-\mathrm{S}=10^{\circ} \mathrm{S} \mathrm{RL}$ & $084^{\circ} / 69^{\circ} \mathrm{S}-\mathrm{S}=78^{\circ} \mathrm{W}$ \\
\hline & & & & & & $053^{\circ} / 82^{\circ} \mathrm{N}-\mathrm{S}=0^{\circ}$ & $039^{\circ} / 82^{\circ} \mathrm{N}-\mathrm{S}=0^{\circ}$ & $162^{\circ} / 80^{\circ} \mathrm{E}-\mathrm{S}=003^{\circ} \mathrm{S}$ \\
\hline & & & & & & $105^{\circ} / 55^{\circ} \mathrm{N}-\mathrm{S}=016^{\circ} \mathrm{E}$ & $103^{\circ} / 34^{\circ} \mathrm{S}-\mathrm{S}=09^{\circ} \mathrm{E}$ & $098^{\circ} / 55^{\circ} \mathrm{S}-\mathrm{S}=02^{\circ} \mathrm{E}$ \\
\hline & & & & & & $128^{\circ} / 76^{\circ} \mathrm{N}-\mathrm{S}=20^{\circ} \mathrm{S}$ & $128^{\circ} / 49^{\circ} \mathrm{N}-\mathrm{S}=45^{\circ} \mathrm{S}$ & $000^{\circ} / 82^{\circ} \mathrm{E}-\mathrm{S}=18^{\circ} \mathrm{N} \mathrm{RL}$ \\
\hline & & & & & & $055^{\circ} / 80^{\circ} \mathrm{W}-\mathrm{S}=12^{\circ} \mathrm{S}$ & $211^{\circ} / 78^{\circ} \mathrm{W}-\mathrm{S}=43^{\circ} \mathrm{S}$ & $191^{\circ} / 78^{\circ} \mathrm{W}-\mathrm{S}=58^{\circ} \mathrm{S}$ \\
\hline & & & & & & $020^{\circ} / 89^{\circ} \mathrm{W}-\mathrm{S}=35^{\circ} \mathrm{S}$ & & \\
\hline & & & & & & \multicolumn{3}{|c|}{$\begin{array}{l}\text { All the above measurements were made in medium-grained graywacke (Matanuska Formation). The Tertiary volcanics on } \\
\text { the other side of the valley had abundant shear fractures, but very few good slickensides. }\end{array}$} \\
\hline $79-46$ & F79-46 & & 6 & 440294 & 6858177 & \multicolumn{3}{|c|}{$\begin{array}{l}\text { Elevation: 4,630 feet } \\
\text { Sample F79-46 - Dacite for Rb, Sr, Ba. [Determined to be dacite by geochemical whole-rock analysis done after dissertation } \\
\text { completed. See analyses.] }\end{array}$} \\
\hline $79-47$ & F79-47 & & 6 & 440228 & 6858145 & \multicolumn{3}{|c|}{$\begin{array}{l}\text { Sample F79-47 - Dacite (two bags) for age dating and Rb, Sr, Ba. [Determined to be dacite by geochemical whole-rock analysis } \\
\text { done after dissertation completed. See analyses.] }\end{array}$} \\
\hline $79-48$ & F79-48 & $\begin{array}{l}\text { North of } \\
\text { Caribou fault }\end{array}$ & 6 & 445863 & 6869325 & \multicolumn{3}{|c|}{$\begin{array}{l}\text { Sample F79-48 - Siltstone for palynology (\#32393) by ARCO (J.E. Bennett). Upper Jurassic (W.A. Fuchs: therefore Chinitna } \\
\text { Formation); see Fuchs dissertation for report. }\end{array}$} \\
\hline $79-49$ & F79-49 & $\begin{array}{l}\text { North of } \\
\text { Caribou fault }\end{array}$ & 6 & 441993 & 6867629 & \multicolumn{3}{|c|}{$\begin{array}{l}\text { Sample F79-49 - Siltstone for palynology (\#32394) by ARCO (J.E. Bennett). Upper Jurassic (W.A. Fuchs: therefore Chinitna } \\
\text { Formation); see Fuchs dissertation for report. }\end{array}$} \\
\hline $79-50$ & F79-50 & $\begin{array}{l}\text { South of } \\
\text { Caribou fault }\end{array}$ & 6 & 434795 & 6864294 & \multicolumn{3}{|c|}{$\begin{array}{l}\text { Sample F79-50 - Shaly siltstone for palynology (\#32395) by ARCO (J.E. Bennett). Upper Cretaceous (Campanian/Maastrichtian). } \\
\text { (W.A. Fuchs: therefore Matanuska Formation); see Fuchs dissertation for report. }\end{array}$} \\
\hline $79-51$ & F79-51 & & 6 & 444881 & 6866015 & \multicolumn{3}{|c|}{$\begin{array}{l}\text { Sample F79-51 - Rhyolite for Rb, Sr, Ba. [Determined to be rhyolite by geochemical whole-rock analysis done after disserta- } \\
\text { tion completed. See analyses.] }\end{array}$} \\
\hline $79-52$ & F79-52 & $\begin{array}{l}\text { North of } 14 \text { mile } \\
\text { on Chickaloon } \\
\text { River }\end{array}$ & 6 & 431916 & 6866307 & \multicolumn{3}{|c|}{$\begin{array}{l}\text { Elevation: 3,550 to 3,600 feet } \\
\text { Sample F79-52 - Three samples (two samples pebble-cobble conglomerate, one sample coarse graywacke) }\end{array}$} \\
\hline $79-53$ & F79-53 & $\begin{array}{l}\text { West of Hicks } \\
\text { Creek }\end{array}$ & 6 & 450460 & 6857088 & \multicolumn{3}{|c|}{$\begin{array}{l}\text { Sample F79-53 - Rhyolite for } \mathrm{Rb}, \mathrm{Sr}, \mathrm{Ba} \text {. [Determined to be rhyolite by geochemical whole-rock analysis done after disserta- } \\
\text { tion completed. See analyses.] }\end{array}$} \\
\hline
\end{tabular}


CASTLE MOUNTAIN - CARIBOU FAULT SYSTEM FIELD NOTES (referenced to station localities - UTM NAD27 CONUS)

by William A. Fuchs

\begin{tabular}{|c|c|c|c|c|c|c|}
\hline NOTE & SAMPLE & LOCATION & ZN & EASTING & NORTHING & DESCRIPTION \\
\hline $79-54$ & F79-54 & & 6 & 450350 & 6857404 & $\begin{array}{l}\text { Elevation: 4,670 feet } \\
\text { Sample F79-54 - Dacite for Rb, Sr, Ba. Note: } 20 \text { foot? dike which goes right into main body to west; quartz phenocrysts. } \\
\text { [Determined to be dacite by geochemical whole-rock analysis done after dissertation completed. See analyses.] }\end{array}$ \\
\hline $79-55$ & F79-55 & & 6 & 449827 & 6857059 & $\begin{array}{l}\text { Elevation: 4,510 feet } \\
\text { Sample F79-55 - Dacite; nearby diabase dike ( } 2 \text { feet thick). Trends } \mathrm{N} 60^{\circ} \mathrm{W} \text {. [Determined to be dacite by geochemical whole- } \\
\text { rock analysis done after dissertation completed. See analyses.] }\end{array}$ \\
\hline $79-56$ & F79-56 & $\begin{array}{l}\text { Slightly uphill } \\
\text { and to west of } \\
79-55\end{array}$ & 6 & 449784 & 6857094 & $\begin{array}{l}\text { Sample F79-56 - Dacite; slightly different lithology from F79-55, country rock inclusions; jasper uphill from here. [Determined } \\
\text { to be dacite by geochemical whole-rock analysis done after dissertation completed. See analyses.] }\end{array}$ \\
\hline $79-57$ & F79-57 & & 6 & 447756 & 6856293 & $\begin{array}{l}\text { Elevation: } 4,760 \text { feet } \\
\text { Sample F79-57 - Chickaloon sandstone (not felsic intrusive as mapped by Detterman and others [1976]); } 20 \text { feet thick; dip } \\
60^{\circ} \text { in direction } 007^{\circ}\end{array}$ \\
\hline $79-58$ & F79-58 & & 6 & 447695 & 6856307 & $\begin{array}{l}\text { Elevation: 4,810 feet } \\
\text { Sample F79-58 - Chickaloon sandstone (not felsic intrusive as mapped by Detterman and others [1976]) }\end{array}$ \\
\hline $79-59$ & F79-59 & $\begin{array}{l}\text { Northeast of } \\
\text { thrust up Doone } \\
\text { Creek }\end{array}$ & 6 & 423043 & 6861697 & Sample F79-59 (three bags) - Talkeetna Formation agglomerate or coarse tuff, for age dating. \\
\hline $79-60$ & & & 6 & 442048 & 6862089 & $\begin{array}{l}\text { Elevation: 3,130 feet } \\
\text { Diabase with coarse feldspar phenocrysts; small sheeted dike complex; probably Tertiary. Note: It is Tertiary because of latite } \\
\text { xenoliths in the gabbro, found upstream from this locality and in the second canyon to the west. }\end{array}$ \\
\hline $79-61$ & & & 6 & 442472 & 6861382 & $\begin{array}{l}\text { Major cataclastic fault zone, trends } \sim 100^{\circ} \text { (Mesozoic fault?), occupied in places by strange dikes of unknown age. Entire zone } \\
\text { probably } 200 \text { feet wide. Zone appears to have steep northerly dip. From what I can see of it, the fault is entirely in Talkeetna } \\
\text { Formation. }\end{array}$ \\
\hline $79-62$ & & & 6 & 442681 & 6861035 & $\begin{array}{l}\text { Elevation: 3,990 feet } \\
\text { Small cataclastic fault zone, vertical, subhorizontal slickensides }\end{array}$ \\
\hline $79-63$ & & & 6 & 443165 & 6860818 & $\begin{array}{l}\text { Elevation: 4,520 feet } \\
\text { Small marble pod, elliptical } 200 \times 100 \text { feet, calcite mainly in veins; it appears to be a possible candidate for the limestone } \\
\text { unit of the Talkeetna Formation (north of Castle Mountain-Caribou fault), but it is impossible to say for sure; the marble is } \\
\text { mixed with volcanics, so it would have had to be extensively remobilized. }\end{array}$ \\
\hline $79-64$ & F79-64 & & 6 & 443509 & 6860507 & $\begin{array}{l}\text { Elevation: 5,110 feet } \\
\text { Sample F79-64 - Dacite for Rb, Sr, Ba analysis; *hand specimen sample contains a rod-shaped zircon within black circle. } \\
\text { [Determined to be dacite by geochemical whole-rock analysis done after dissertation completed. See analyses.] }\end{array}$ \\
\hline $79-65$ & F79-65 & & 6 & 443184 & 6860202 & $\begin{array}{l}\text { Elevation: 5,150 feet } \\
\text { Sample F79-65 - Dacite for Rb, Sr, Ba analysis. [Determined to be dacite by geochemical whole-rock analysis done after } \\
\text { dissertation completed. See analyses.] }\end{array}$ \\
\hline $79-66$ & & & 6 & 442651 & 6861679 & $\begin{array}{l}\text { Elevation: 3,150 feet } \\
\text { Highly-chewed-up gabbro }\end{array}$ \\
\hline $79-67$ & & & 6 & 442755 & 6862258 & $\begin{array}{l}\text { Elevation: } 3,210 \text { feet } \\
\text { Bedding } 186^{\circ} / 20^{\circ} \mathrm{W} \text {. Volcanics - flows and agglomerates - Talkeetna Formation }\end{array}$ \\
\hline $79-68$ & F79-68 & & 6 & 441231 & 6862234 & $\begin{array}{l}\text { Elevation: 3,400 feet } \\
\text { Sample F79-68 - Rhyolite for Rb, Sr, Ba analysis. [Determined to be rhyolite by geochemical whole-rock analysis done after } \\
\text { dissertation completed. See analyses.] }\end{array}$ \\
\hline
\end{tabular}

$$
\text { Page } 44
$$


CASTLE MOUNTAIN - CARIBOU FAULT SYSTEM FIELD NOTES (referenced to station localities - UTM NAD27 CONUS)

by William $A$. Fuchs

\begin{tabular}{|c|c|c|c|c|c|c|}
\hline NOTE & SAMPLE & LOCATION & ZN & EASTING & NORTHING & DESCRIPTION \\
\hline $79-69$ & & & 6 & 441229 & 6862322 & $\begin{array}{l}\text { Elevation: } 3,760 \text { feet } \\
\text { Bedding } 273^{\circ} / 28^{\circ} \mathrm{N} \text {; flow of Talkeetna Formation; coarse tuff in vicinity }\end{array}$ \\
\hline $79-70$ & F79-70 & & 6 & 441115 & 6862214 & $\begin{array}{l}\text { Elevation: 3,440 feet } \\
\text { Sample F79-70 - Rhyolite for Rb, Sr, Ba analysis. [Determined to be rhyolite by geochemical whole-rock analysis done after } \\
\text { dissertation completed. See analyses.] 20-foot-wide basic dike of unknown type located } 25 \text { feet to the south. }\end{array}$ \\
\hline $79-71$ & F79-71 & & 6 & 442059 & 6859060 & $\begin{array}{l}\text { Elevation: } 4,430 \text { feet } \\
\text { Sample F79-71 - Agglomerate for age dating, should represent the oldest Talkeetna Formation I could obtain; not too pristine, } \\
\text { but the best I could do in this area. }\end{array}$ \\
\hline $79-72$ & & & 6 & 441893 & 6859002 & $\begin{array}{l}\text { Elevation: 4,470 feet } \\
\text { Bedding } 177^{\circ} / 32^{\circ} \mathrm{W} \text {; Talkeetna Formation; tuffaceous, fossiliferous (clams), sandstone, medium-grained. }\end{array}$ \\
\hline $79-73$ & $\begin{array}{l}\text { F79-73I } \\
\text { F79-73II }\end{array}$ & & 6 & 441456 & 6860299 & $\begin{array}{l}\text { Cataclasite, large zone(s) extends from here north along side of ridge. Has some similarities to a flat-lying thrust fault in } \\
\text { form, but I basically do not know what is going on here. This may represent a Mesozoic deformational event. It might also be } \\
\text { hydrothermal in large part (note: shot through with a large amount of calcitic, talcose white veinlets, and stringers) and } \\
\text { occurred shortly after deformation. Sample F79-73I- small bit of veinlet material (x-ray might establish PT conditions). } \\
\text { Sample F79-73II - root fragments in cataclasite, some sort of flow? }\end{array}$ \\
\hline $79-74$ & F79-74 & & 6 & 443708 & 6858801 & $\begin{array}{l}\text { Elevation: 4,700 feet } \\
\text { Sample F79-74 - Dacite for Rb, Sr, Ba analysis. [Determined to be dacite by geochemical whole-rock analysis done after } \\
\text { dissertation completed. See analyses.] }\end{array}$ \\
\hline $79-75$ & F79-75 & & 6 & 444968 & 6857340 & $\begin{array}{l}\text { Elevation: 5,730 feet } \\
\text { Sample F79-75 - Calcite from Castle Mountain splay fault for thermoluminescence dating. Found in float in gully just } 90 \text { feet } \\
\text { (elevation-wise) down from fault saddle. Calcite is clearly coming out of fault zone. }\end{array}$ \\
\hline $79-76$ & F79-76 & & 6 & 445371 & 6857661 & $\begin{array}{l}\text { Elevation: 5,250 feet } \\
\text { Sample F79-76 - Dacite/granodiorite (coarse-grained), for thin section analysis of coarse-grained stuff; no geochem. }\end{array}$ \\
\hline $79-77$ & & & 6 & 442260 & 6862335 & $\begin{array}{l}\text { Elevation: 3,690 feet } \\
\text { Gabbro, top contact. Looking across stream to west it can be seen that gabbro was injected in dike-like form and is cut by } \\
\text { diabase dikes, which I have assumed is equivalent to gabbro. Thus, there appear to have been two pulses of magmatic activity } \\
\text { probably of at least slightly different ages. }\end{array}$ \\
\hline $79-78$ & & & 6 & 442308 & 6862451 & $\begin{array}{l}\text { Elevation: 3,990 feet } \\
\text { Volcaniclastic conglomerate; Talkeetna Formation. From gabbro contact to top of slope consists of a bedded marine(?) } \\
\text { sequence of tuff, tuffaceous sandstone, limestone, and volcaniclastic sandstone, no fossils observed. }\end{array}$ \\
\hline $79-79$ & F79-79 & & 6 & 444858 & 6860050 & $\begin{array}{l}\text { Elevation: } 5,270 \text { feet } \\
\text { Sample F79-79 - Dacite for Rb, Sr, Ba analysis. [Determined to be dacite by geochemical whole-rock analysis done after } \\
\text { dissertation completed. See analyses.] }\end{array}$ \\
\hline $79-80$ & F79-80 & & 6 & 439048 & 6859632 & $\begin{array}{l}\text { Elevation: } 3,450 \text { feet } \\
\text { Sample F79-80 -Felsite, for thin section analysis. This stuff is similar to that at B-121, across the way, in that it is different } \\
\text { from normal felsite (rhyolite-dacite) and may not be equivalent. It is phaneritic and has a higher mafic content than normal } \\
\text { felsite, but in the end it is probably equivalent. }\end{array}$ \\
\hline $79-81$ & & & 6 & 440530 & 6857833 & $\begin{array}{l}\text { Elevation: } 4,610 \text { feet } \\
\text { Bedding } 084^{\circ} / 27^{\circ} \mathrm{S} \text { in Matanuska Formation, argillite. }\end{array}$ \\
\hline
\end{tabular}


CASTLE MOUNTAIN - CARIBOU FAULT SYSTEM FIELD NOTES (referenced to station localities - UTM NAD27 CONUS)

by William A. Fuchs

\begin{tabular}{|c|c|c|c|c|c|c|}
\hline NOTE & SAMPLE & LOCATION & ZN & EASTING & NORTHING & DESCRIPTION \\
\hline $79-82$ & F79-82 & & 6 & 438975 & 6858992 & $\begin{array}{l}\text { Sample F79-82 - Shaly material for micropaleontology (but never analyzed), Tuxedni(?) Group. Marine because sandstone } \\
\text { bed above contain pelecypods, abundant plant fragments in units above, whole unit is brownish with not highly indurated } \\
\text { sandstones, siltstone, shales; bedding } 40 \text { feet up from sample } 184^{\circ} / 30^{\circ} \mathrm{W} \text {; if not Tuxedni Group, may be Matanuska Forma- } \\
\text { tion (or Talkeetna?) unit estimated to be } 200 \text { feet thick. }\end{array}$ \\
\hline $79-83$ & & & 6 & 440386 & 6858803 & $\begin{array}{l}\text { Elevation: 4,750 feet } \\
\text { Tuxedni(?) Group, estimated } 30 \text { feet thick underlying Matanuska Formation; sandstone contains abundant mollusks, belem- } \\
\text { nites, trigonia, and one beautiful small ammonite that was collected. Too small to map. Tuxedni Group appears to be thin- } \\
\text { ning to the southeast. }\end{array}$ \\
\hline $79-84$ & & & 6 & 440459 & 6858657 & $\begin{array}{l}\text { Elevation: 4,670 feet } \\
\text { Matanuska Formation, medium-grained sandstone. Bedding } 200^{\circ} / 27^{\circ} \mathrm{W}\end{array}$ \\
\hline $79-85$ & F79-85 & & 6 & 444517 & 6863505 & $\begin{array}{l}\text { Elevation: 4,430 feet } \\
\text { Sample F79-85 - Rhyolite for Rb, Sr, Ba analysis. [Determined to be rhyolite by geochemical whole-rock analysis done after } \\
\text { dissertation completed. See analyses.] }\end{array}$ \\
\hline $79-86$ & F79-86 & & 6 & 450003 & 6864222 & $\begin{array}{l}\text { Elevation: 5,520 feet } \\
\text { Sample F79-86 - Felsic intrusive from talus at the base of the pinnacles. This sample is an example of abundant coarse-grained } \\
\text { material; for potential thin section analysis; no geochem. }\end{array}$ \\
\hline
\end{tabular}

\section{ACKNOWLEDGEMENTS}

Map digitally redrafted by Jo Beth Allen

Indispensable contributions of field assistants Miriam Hill, Donna Hewitt, Steve McMillin, and Victoria Schurer

Reviewer Robert J. Gillis, Alaska Division of Geological \& Geophysical Surveys

Numerous others are acknowledged in the dissertation

\section{REFERENCES}

Detterman, R.L., Plafker, George, Tysdal, R.B., and Hudson, Travis, 1976, Geology and surface features along the Castle Mountain fault system, Alaska: U.S. Geological Survey Miscellaneous Field Studies Map MF-738, 1 sheet, scale 1:63,360. http://dggs.alaska.gov/pubs/id/13295

Fuchs, W.A., 1980, Tertiary tectonic history of the Castle Mountain-Caribou fault system in the Talkeetna Mountains, Alaska: University of Utah, Salt Lake City, Ph.D. dissertation, 162 p., illust. (some color), maps. http://dggs.alaska.gov/pubs/id/27944 


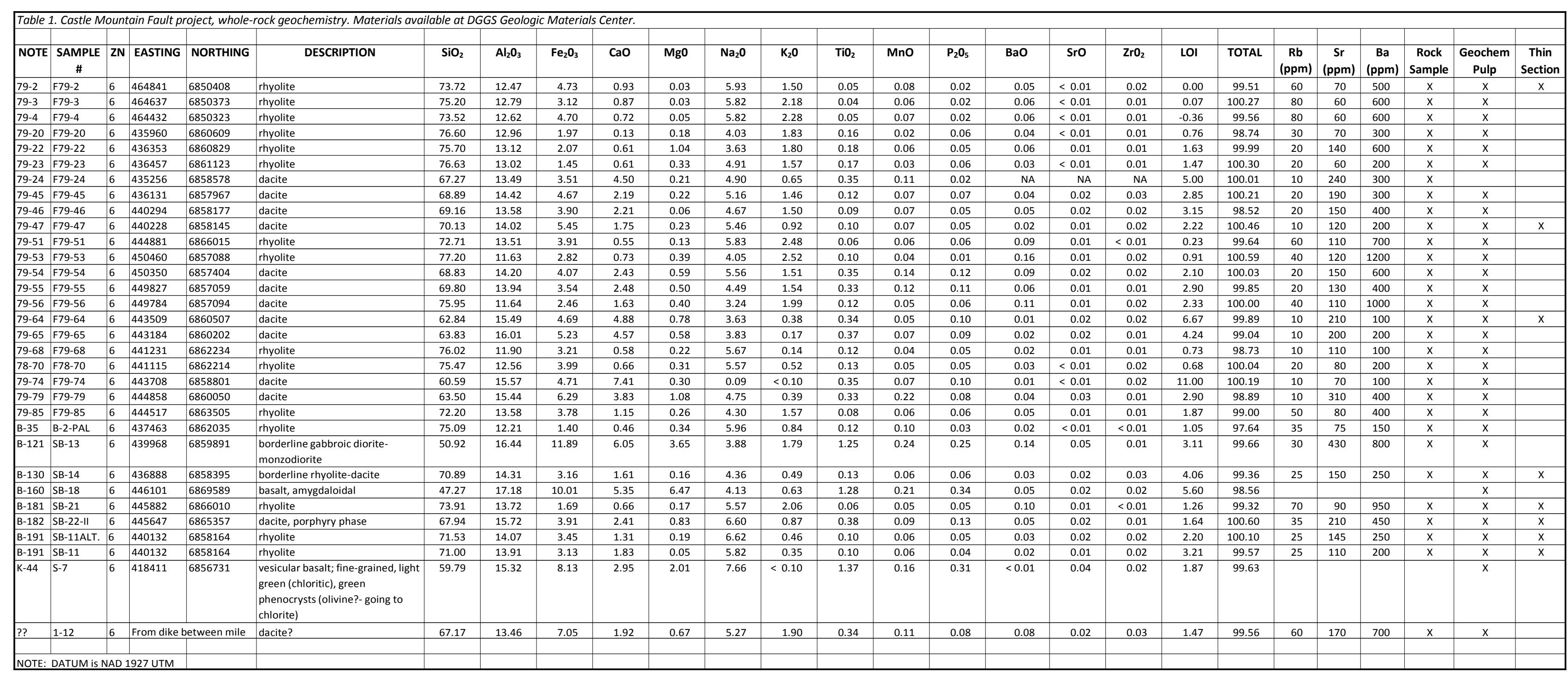


To50

YOUR REFEREMCE: CALCULATE FED \& FE2OB

\begin{tabular}{|c|c|c|c|c|c|c|c|c|c|c|c|c|c|c|c|}
\hline SAMPLE & WDOZ & A.1203 & $F=203$ & $\mathrm{CaO}$ & $\operatorname{Mg} 0$ & $\mathrm{~N}=20$ & KEO & Ti02 & Mno & P205 & $8 a 0$ & $5 \mathrm{ro}$ & $\mathrm{ZrOZ}$ & $\operatorname{LOT}$ & TOTAL \\
\hline $\begin{array}{l}F-79-2 \\
F-79-3 \\
F-79-4 \\
F-79-20 \\
F-79-22\end{array}$ & $\begin{array}{l}73.72 \\
75.20 \\
73.52 \\
76.60 \\
75.70\end{array}$ & $\begin{array}{l}12.47 \\
12.79 \\
12.82 \\
12.96 \\
13.12\end{array}$ & $\begin{array}{l}5.73 \\
3.12 \\
4.70 \\
1.97 \\
2.07\end{array}$ & $\begin{array}{l}.93 \\
.87 \\
.72 \\
.13 \\
.61\end{array}$ & $\begin{array}{r}.03 \\
.03 \\
.05 \\
.18 \\
1.04\end{array}$ & $\begin{array}{l}5.93 \\
5.62 \\
5.62 \\
4.03 \\
3.63\end{array}$ & $\begin{array}{l}1.50 \\
2.18 \\
2.28 \\
1.83 \\
1.80\end{array}$ & $\begin{array}{l}.05 \\
.04 \\
.05 \\
.15 \\
.18\end{array}$ & $\begin{array}{l}.06 \\
.08 \\
.07 \\
.02 \\
.06\end{array}$ & $\begin{array}{l}.02 \\
.02 \\
.02 \\
.06 \\
.05\end{array}$ & $\begin{array}{l}.05 \\
.08 \\
.08 \\
.04 \\
.05\end{array}$ & $\begin{array}{l}<.02 \\
<.01 \\
<.01 \\
.01 \\
.01\end{array}$ & $\begin{array}{l}.02 \\
.01 \\
.01 \\
.01 \\
.01\end{array}$ & $\begin{array}{r}0.00 \\
0.07 \\
-0.38 \\
0.75 \\
1.63\end{array}$ & $\begin{array}{r}90.51 \\
100.27 \\
99.55 \\
98.74 \\
99.99\end{array}$ \\
\hline $\begin{array}{l}F-79-23 \\
F-79-45 \\
F-79-46 \\
F-79-47 \\
F-79-51\end{array}$ & $\begin{array}{l}76.63 \\
68.99 \\
65.16 \\
70.13 \\
72.71\end{array}$ & $\begin{array}{l}13.02 \\
14.42 \\
13.58 \\
14.02 \\
13.51\end{array}$ & $\begin{array}{l}4.45 \\
4.67 \\
3.90 \\
5.45 \\
3.91\end{array}$ & $\begin{array}{r}.61 \\
2.19 \\
2.21 \\
1.75 \\
.55\end{array}$ & $\begin{array}{l}.33 \\
.22 \\
.06 \\
.23 \\
.13\end{array}$ & $\begin{array}{l}4.91 \\
5.16 \\
4.67 \\
5.46 \\
5.93\end{array}$ & $\begin{array}{l}1.57 \\
1.46 \\
1.50 \\
.92 \\
2.48\end{array}$ & $\begin{array}{l}.17 \\
.12 \\
.09 \\
.10 \\
.05\end{array}$ & $\begin{array}{l}.03 \\
.07 \\
.07 \\
.07 \\
.05\end{array}$ & $\begin{array}{l}.06 \\
.07 \\
.05 \\
.05 \\
.05\end{array}$ & $\begin{array}{l}.03 \\
.04 \\
.05 \\
.02 \\
.09\end{array}$ & $\begin{array}{r}.01 \\
.02 \\
.02 \\
.01 \\
.01\end{array}$ & $\begin{array}{r}.01 \\
.03 \\
.02 \\
.02 \\
.01\end{array}$ & $\begin{array}{l}1.47 \\
2.85 \\
3.15 \\
2.22 \\
0.23\end{array}$ & $\begin{array}{r}100.30 \\
100.21 \\
96.52 \\
100.48 \\
99.64\end{array}$ \\
\hline $\begin{array}{l}F-79-53 \\
F-79-54 \\
F-79-55 \\
F-79-56 \\
F-79-94\end{array}$ & $\begin{array}{l}77.20 \\
86.83 \\
69.80 \\
75.95 \\
82.84\end{array}$ & $\begin{array}{l}11.63 \\
14.20 \\
13.94 \\
11.64 \\
15.79\end{array}$ & $\begin{array}{l}2.82 \\
4.07 \\
2.54 \\
2.46 \\
4.09\end{array}$ & $\begin{array}{l}.73 \\
2.43 \\
2.48 \\
1.63 \\
4.88\end{array}$ & $\begin{array}{l}.35 \\
.59 \\
.50 \\
.40 \\
.76\end{array}$ & $\begin{array}{l}4.05 \\
5.58 \\
4.49 \\
3.24 \\
3.33\end{array}$ & $\begin{array}{l}2.52 \\
1.51 \\
2.54 \\
1.99 \\
.38\end{array}$ & $\begin{array}{l}.10 \\
.35 \\
.39 \\
.12 \\
.34\end{array}$ & $\begin{array}{l}.04 \\
.24 \\
.12 \\
.05 \\
.05\end{array}$ & $\begin{array}{l}.01 \\
.12 \\
.12 \\
.06 \\
.10\end{array}$ & $\begin{array}{l}.16 \\
.00 \\
.06 \\
.11 \\
.01\end{array}$ & $\begin{array}{l}.01 \\
.02 \\
.01 \\
.01 \\
.02\end{array}$ & $\begin{array}{l}.02 \\
.02 \\
.01 \\
.01 \\
.02 \\
.02\end{array}$ & $\begin{array}{l}0.91 \\
2.10 \\
2.90 \\
2.33 \\
6.97\end{array}$ & $\begin{array}{r}100.59 \\
100.03 \\
99.85 \\
100.00 \\
99.89\end{array}$ \\
\hline $\begin{array}{l}F-79-65 \\
F-79-69 \\
F-79-70 \\
F-79-74 \\
F-79-79\end{array}$ & $\begin{array}{l}63.63 \\
78.02 \\
75.47 \\
50.59 \\
83.50\end{array}$ & $\begin{array}{l}15.01 \\
11.90 \\
12.56 \\
15.57 \\
15.44\end{array}$ & $\begin{array}{l}5.23 \\
3.21 \\
3.99 \\
4.71 \\
6.29\end{array}$ & $\begin{array}{r}4.57 \\
.59 \\
.86 \\
7.41 \\
3.83\end{array}$ & $\begin{array}{r}.58 \\
.22 \\
.31 \\
.30 \\
. .08\end{array}$ & $\begin{array}{l}3.63 \\
5.67 \\
5.57 \\
.09 \\
4.75\end{array}$ & $\begin{array}{l}.17 \\
.14 \\
.52 \\
.10 \\
.39\end{array}$ & $\begin{array}{l}.37 \\
.12 \\
.13 \\
.35 \\
.33\end{array}$ & $\begin{array}{l}.07 \\
.04 \\
.05 \\
.07 \\
.22\end{array}$ & $\begin{array}{l}.05 \\
.05 \\
.05 \\
.10 \\
.08\end{array}$ & $\begin{array}{l}.02 \\
.02 \\
.03 \\
.01 \\
.04\end{array}$ & $\begin{array}{r}.02 \\
.01 \\
6.01 \\
.01 \\
.03\end{array}$ & $\begin{array}{l}.01 \\
.01 \\
.02 \\
.02 \\
.01\end{array}$ & $\begin{array}{r}4.24 \\
0.73 \\
0.60 \\
14.00 \\
2.90\end{array}$ & $\begin{array}{r}90.09 \\
96.73 \\
100.04 \\
100.19 \\
98.69\end{array}$ \\
\hline
\end{tabular}

T.B.L. REPORT Na: : T $-6500 \cdots$ T.S.L. File No: : WHN25:

Invoice \# 25492 
TECHNICAL SERUICE LABORATURIES

1301 FEHSTER DRIVE, MISSISSALGA, ONTARIO
TELEPHONE: (4I6) 625 -1544

CERTIFICATE OF ANALYSIS

WILLIAM A. FUCHS

1555 Ridgeview Dr. Unit \#B

RENO NEVAZA U.S.A.

89509

YOLR FEFERENEE :

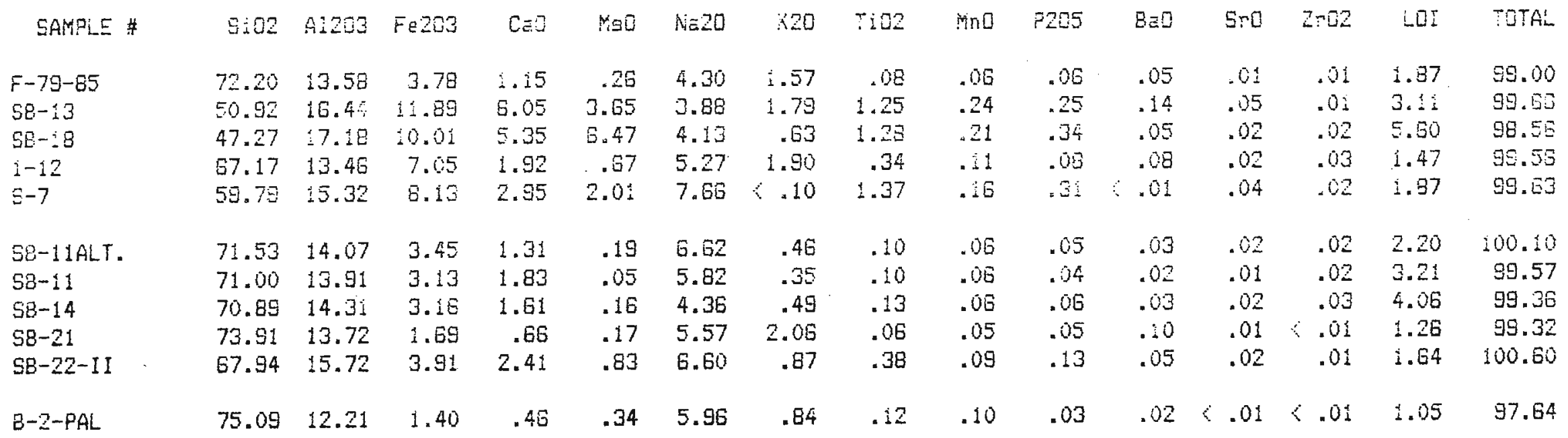

DATE: $25-J 4 N-34$
T.S.L. REPORT VO. : T $T$ - 550 - 2 T.S.L. File No.: : JUN25:

Invoice 非 25492 
F $79-24$

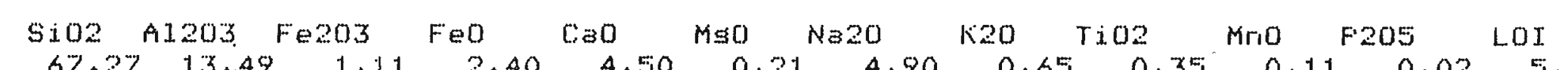

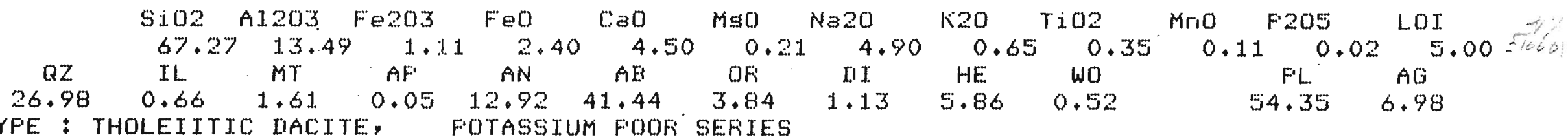

FOCK TYFE: THOLEIITJC IIACITE, FOTASSIUM FOOF SERIES

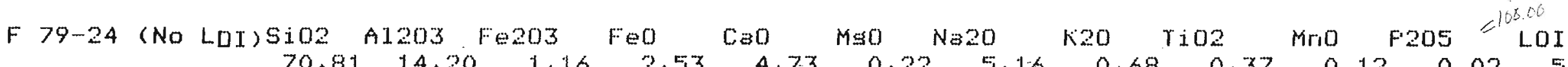

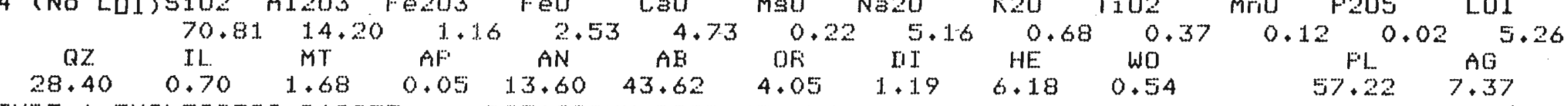

ROCK TYFE: THOLEIITIC IACITE, FOTASSIUM FOOF SEFIES 

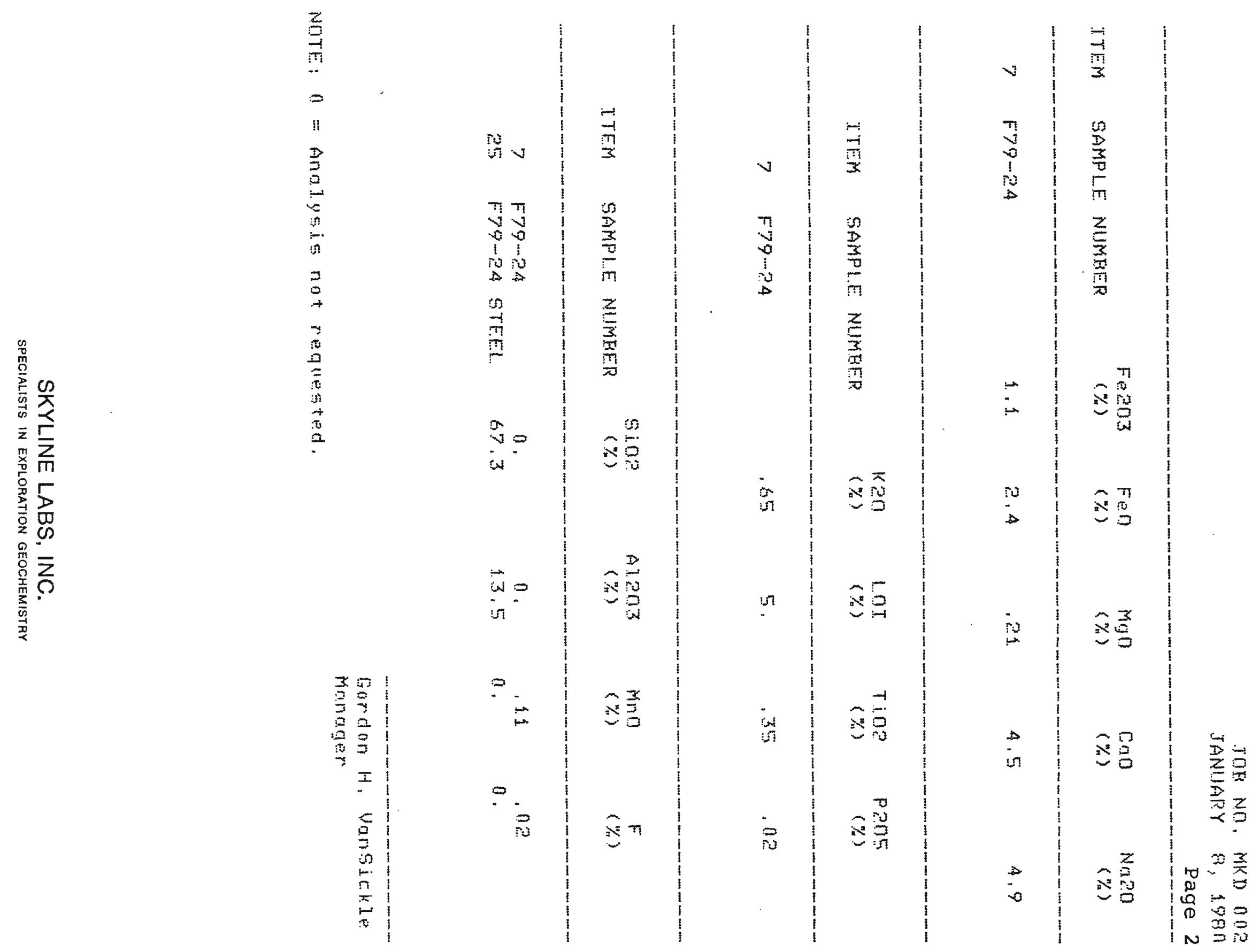

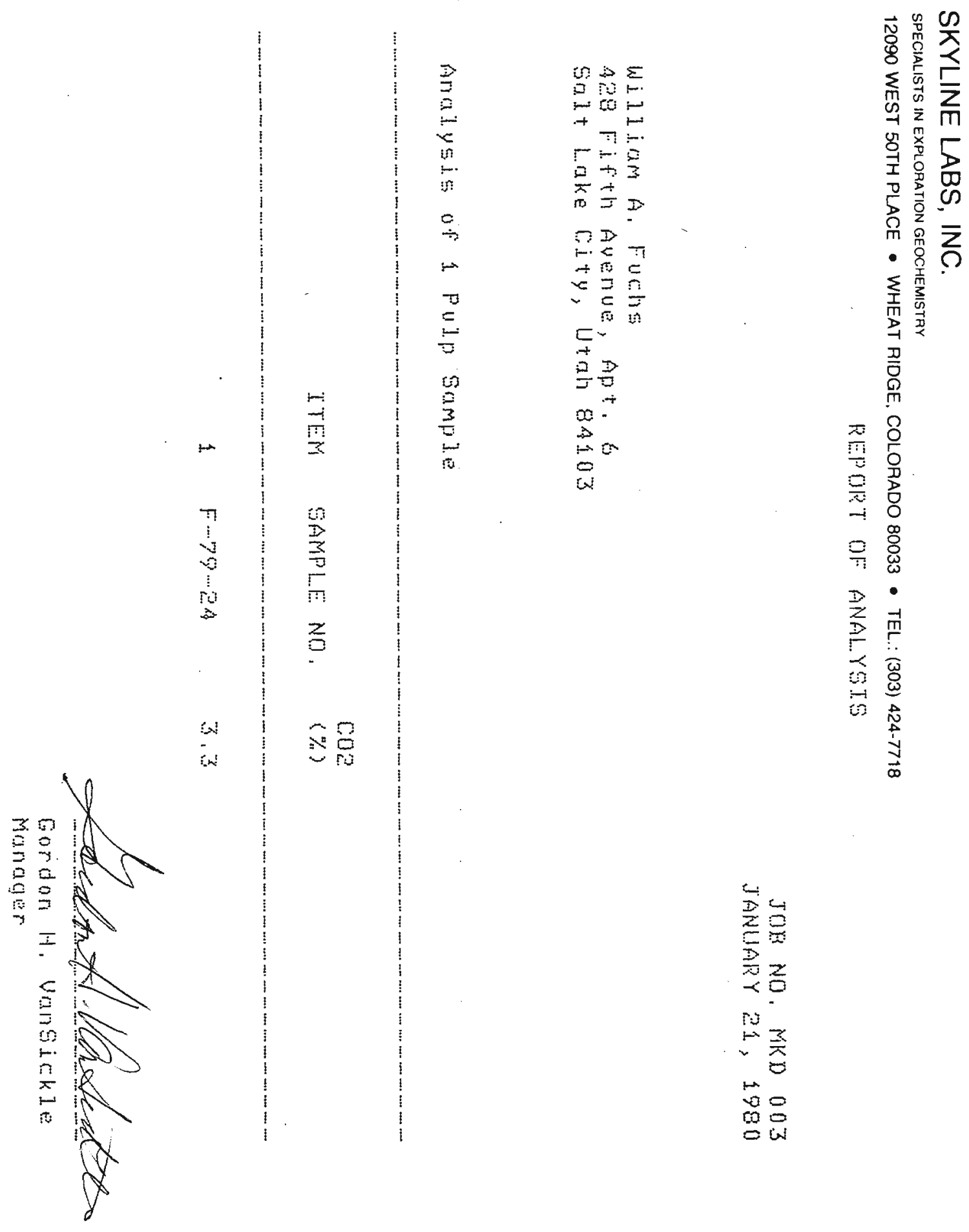

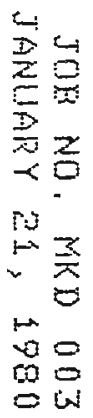




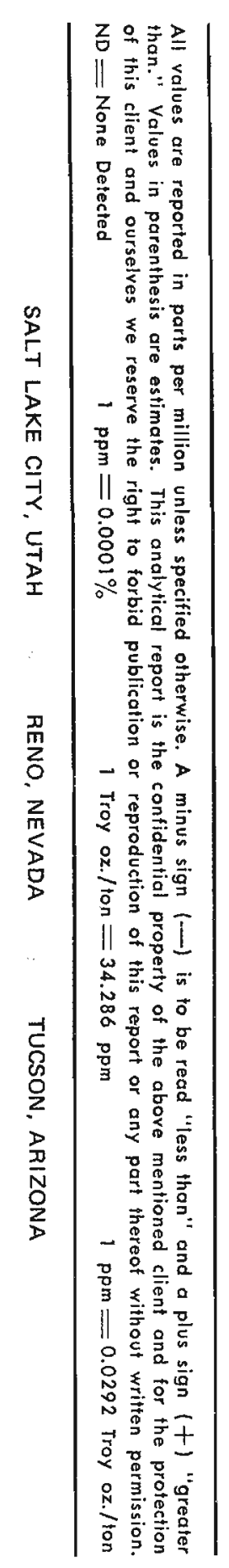

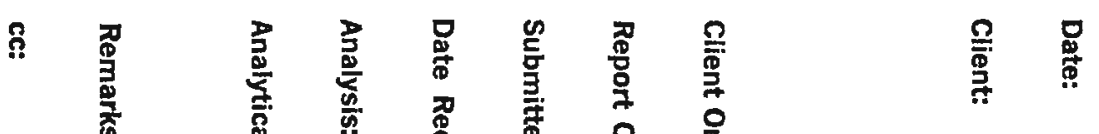

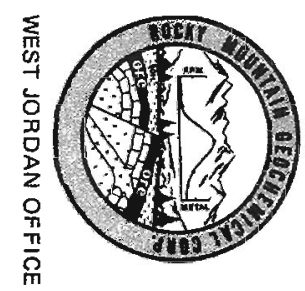

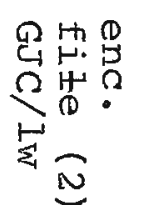
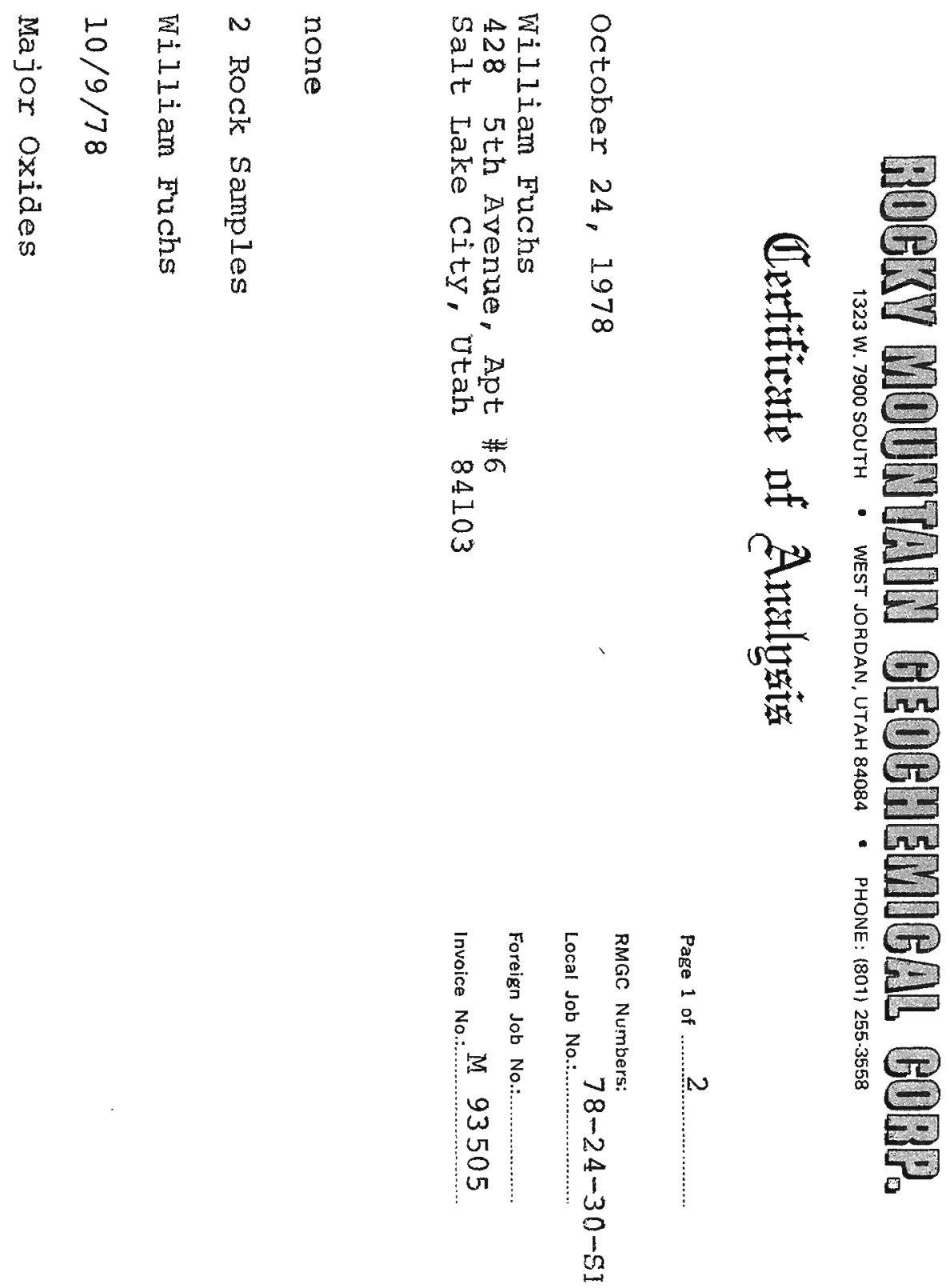


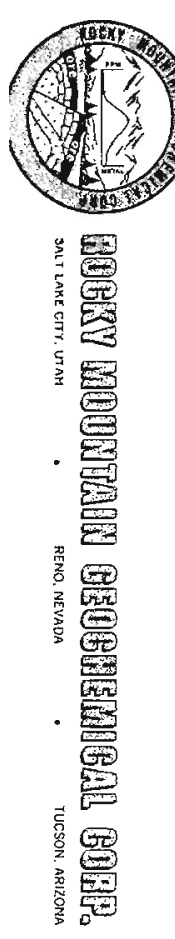

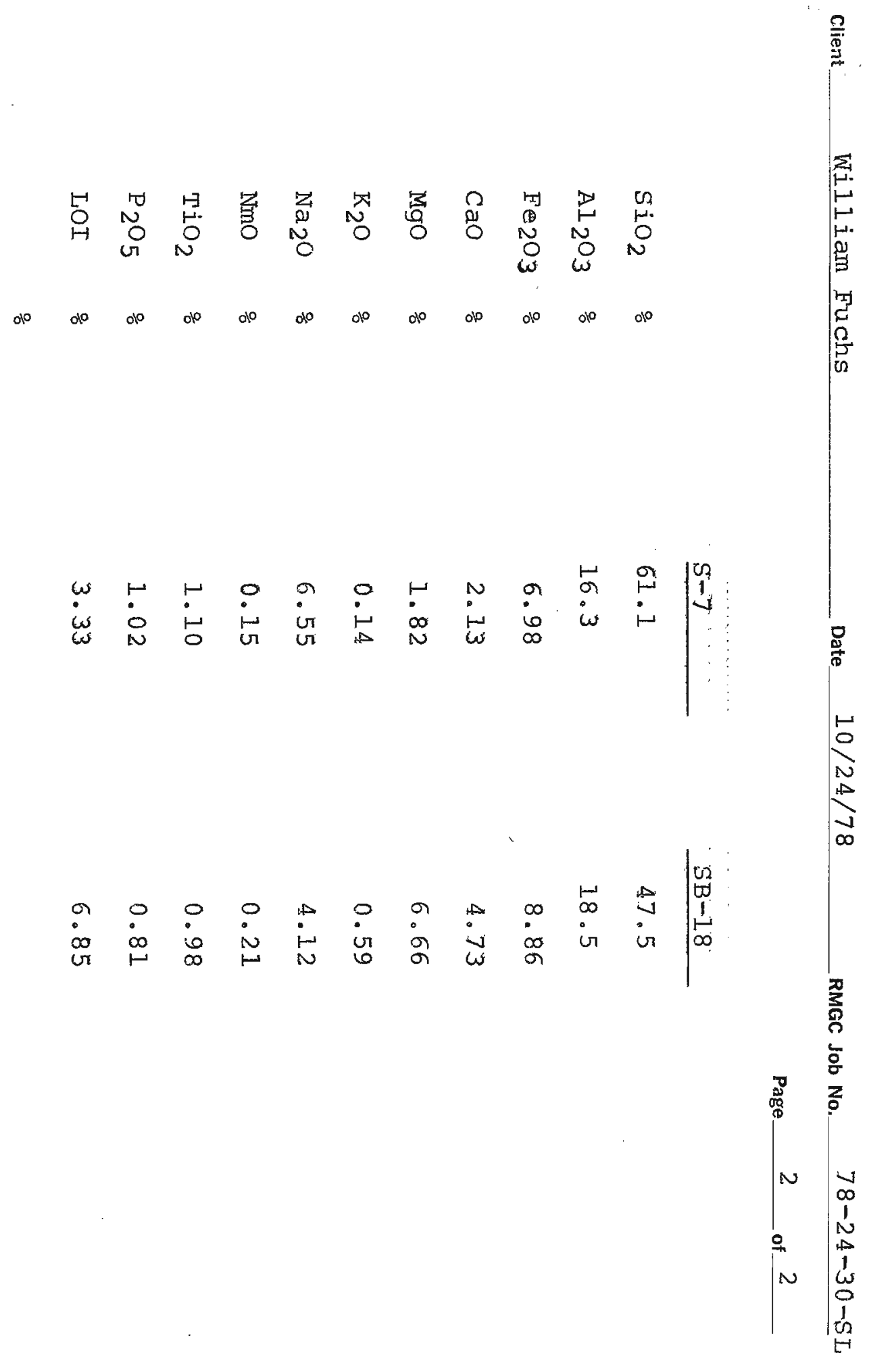


TECHNICAL SERUICE LABQRATURIES

1301 FEKSTER DRIUE, MISSISSAUGA, ONTARIO LAN IA2

TELEPHONE： (416) $825-1544$

CERTIFICATE OF ANALYSIS

WILLIAM A. FLCHS

T.S.L. REPORT NO. : T -8509

T.S.L. File No.: Nín 302

YOUR REFERENCE :

\begin{tabular}{|c|c|c|c|c|c|c|c|c|c|c|c|c|c|c|}
\hline SAMPLE & $5 i 02$ & A1203 & $\mathrm{Fe} 203$ & $\mathrm{CaO}$ & $\operatorname{rag}$ & $\operatorname{Na} 20$ & $\mathrm{~K} 20$ & Tiaz & $\mathrm{MnO}$ & P205 & $\mathrm{BaO}$ & Sro & $2 \mathrm{roz}$ & LOI \\
\hline$B-2-P A L$ & 74.74 & 13.07 & 1.40 & .45 & .35 & 6.50 & .69 & .12 & .11 & .02 & .03 & $<.01$ & $<.01$ & 1.05 \\
\hline
\end{tabular}

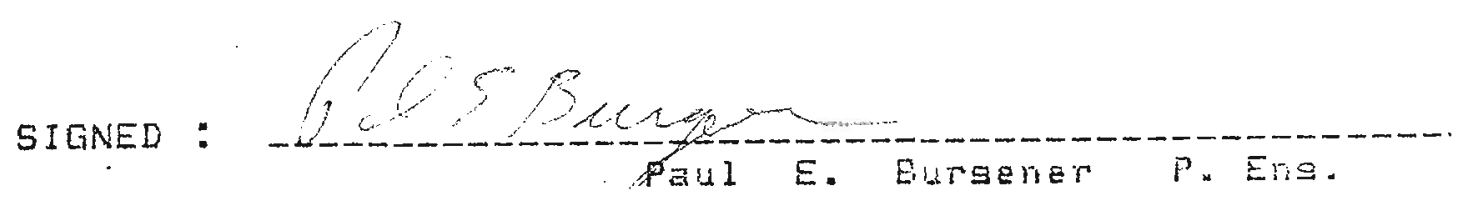




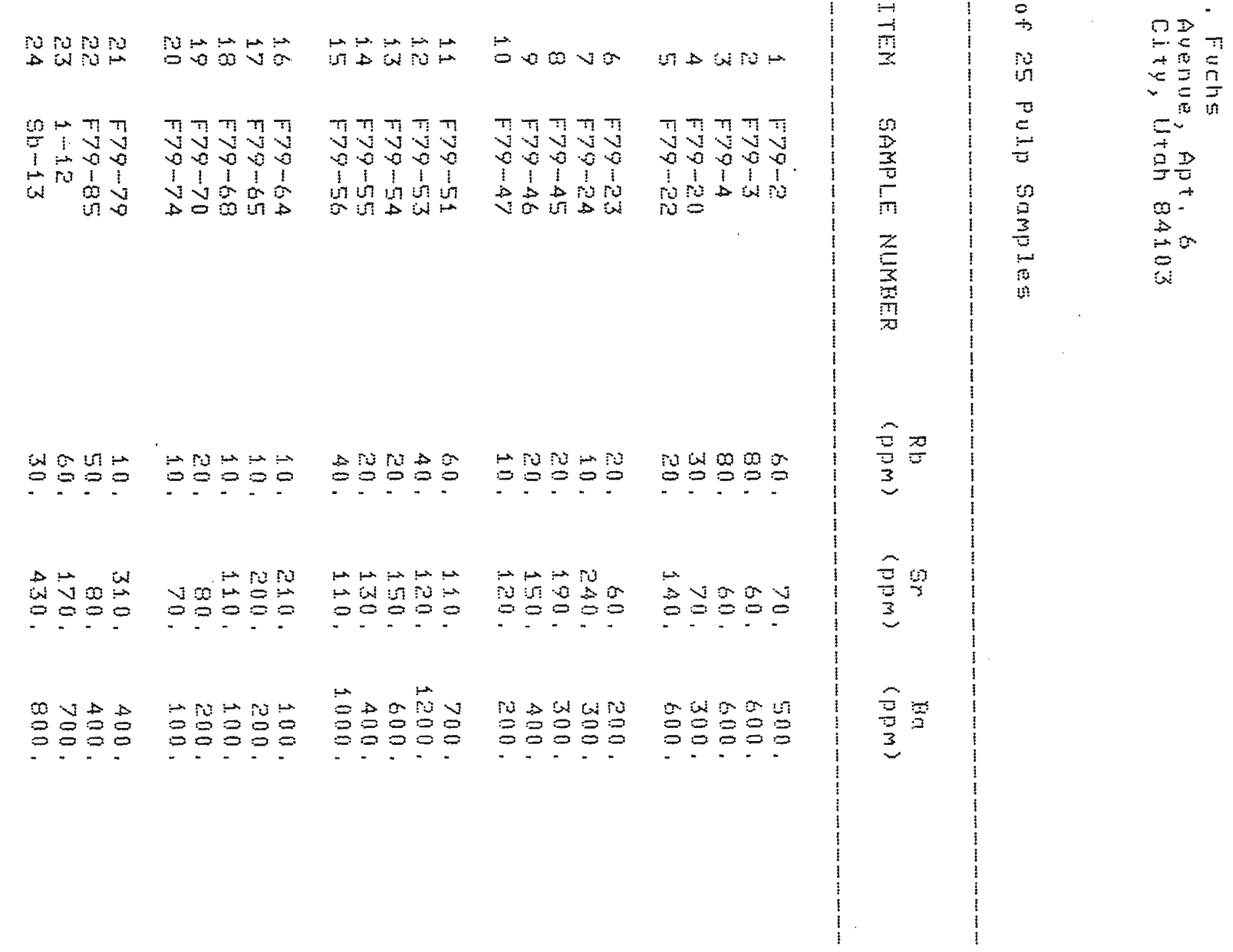




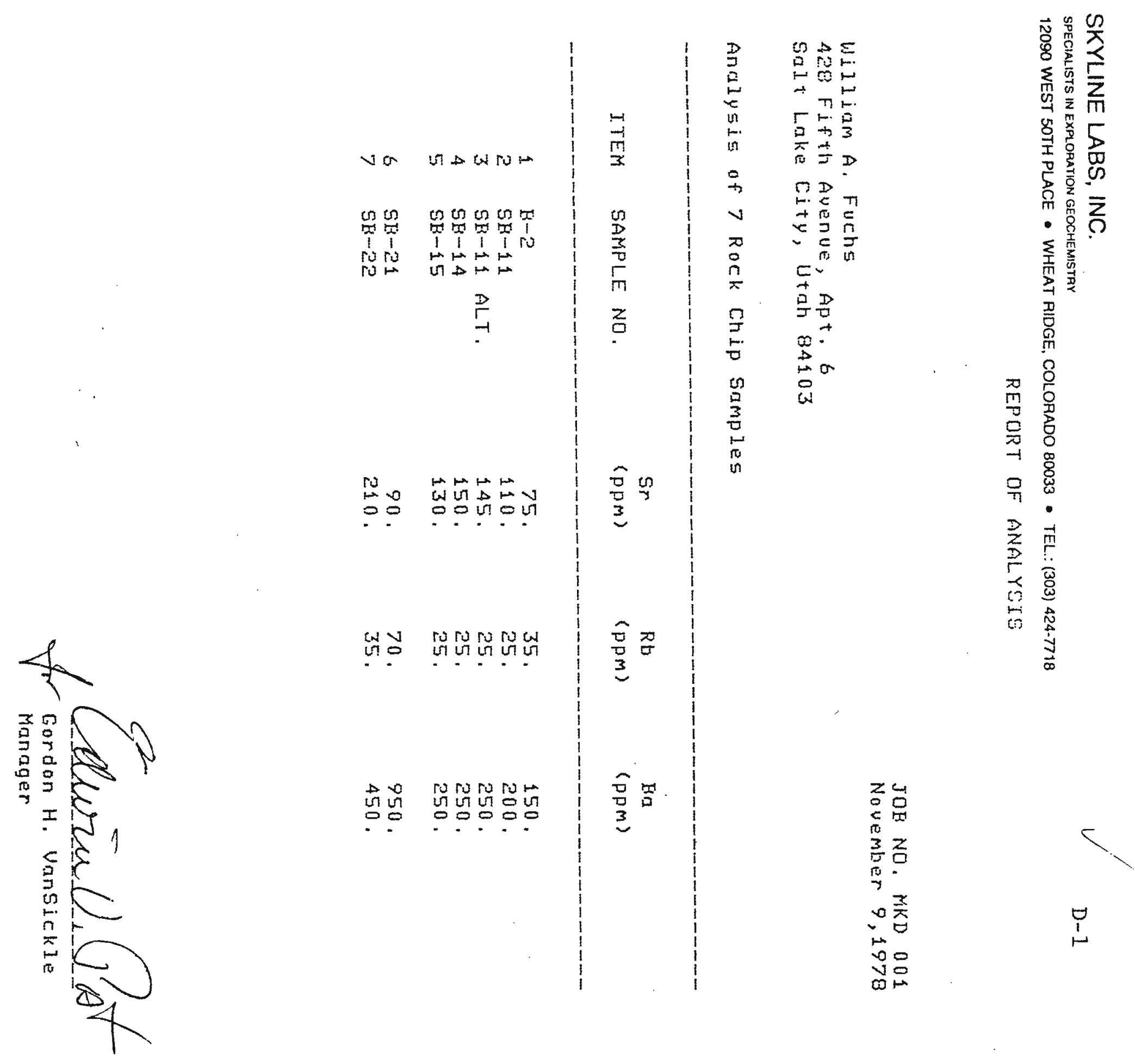




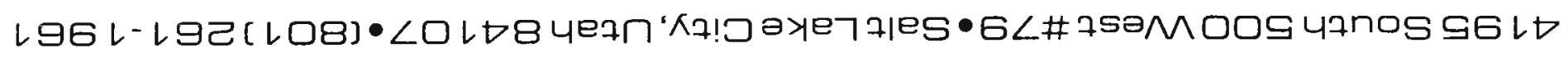
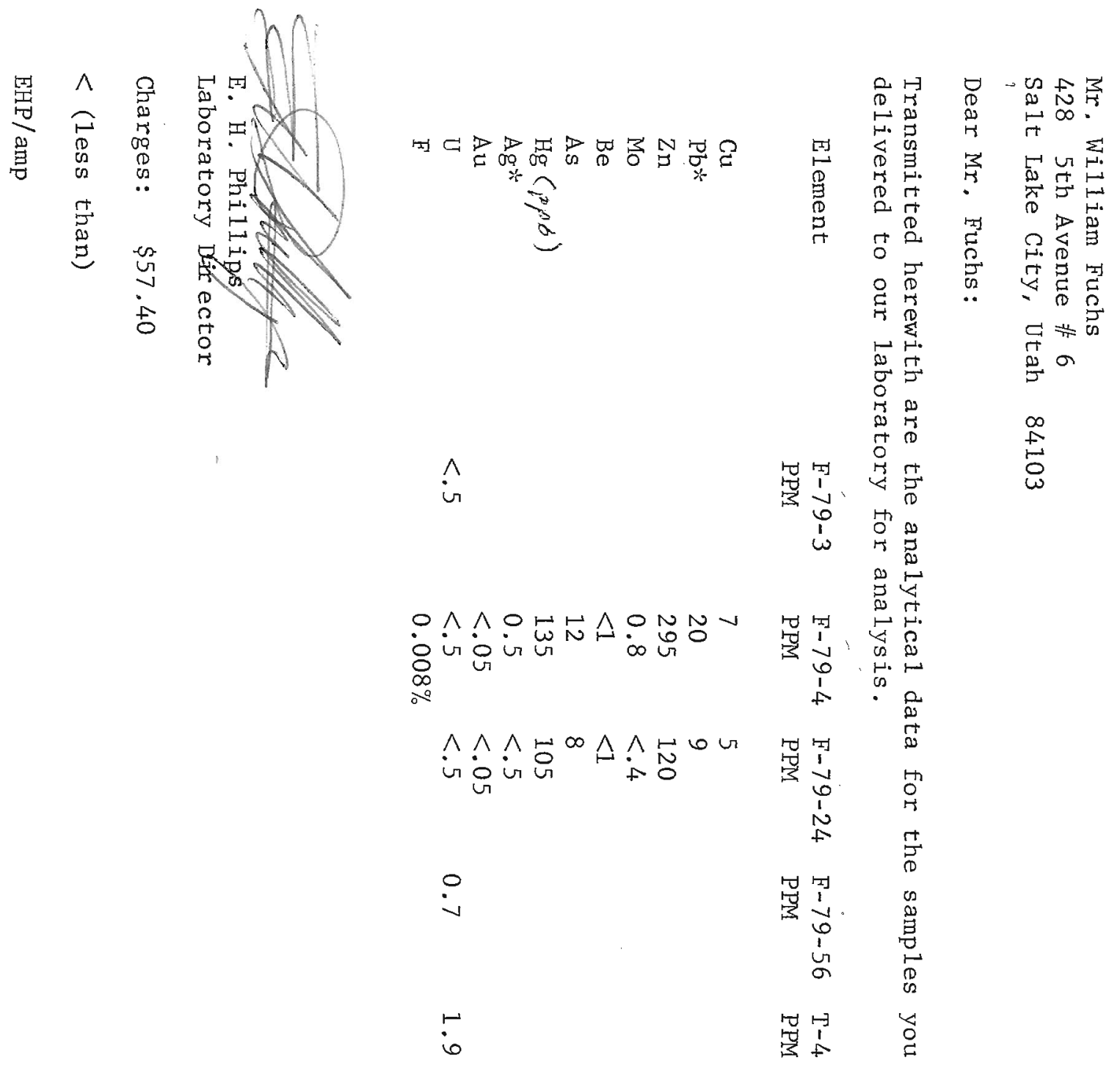